NEANDER ALESSANDRO DA SILVA PAULA

\title{
MPC ADAPTATIVO - MULTIMODELOS PARA CONTROLE DE SISTEMAS NÃO-LINEARES
}




\section{NEANDER ALESSANDRO DA SILVA PAULA}

\section{MPC ADAPTATIVO - MULTIMODELOS PARA CONTROLE DE SISTEMAS NÃO-LINEARES}

Dissertação apresentada à Escola

Politécnica da Universidade de São

Paulo para a obtenção do título de Mestre em Engenharia 
NEANDER ALESSANDRO DA SILVA PAULA

\section{MPC ADAPTATIVO - MULTIMODELOS PARA CONTROLE DE SISTEMAS NÃO-LINEARES}

Dissertação apresentada à Escola Politécnica da Universidade de São Paulo para a obtenção do título de Mestre em Engenharia

Área de Concentração:

Engenharia Química

Orientador:

Prof. Dr. Darci Odloak 


\section{FICHA CATALOGRÁFICA}

Paula, Neander Alessandro da Silva

MPC adaptativo - multimodelos para controle de sistemas näo-lineares / N.A.S. Paula. -- Säo Paulo, 2009.

$123 p$.

Dissertaçăo (Mestrado) - Escola Politécnica da Universidade de Săo Paulo. Departamento de Engenharia Química.

1.Controle adaptativo 2 . Identificação de sistemas 3 . Processos químicos I. Universidade de Săo Paulo. Escola Politécnica. Departamento de Engenharia Química II. t. 


\section{AGRADECIMENTOS}

Agradeço primeiramente a Deus pelo dom da vida e por permitir que tivesse saúde e muita persistência para chegar ao final do trabalho.

Ao prof. Darci Odloak pela orientação e apoio ao longo destes anos pois sem isto não seria possível concluir este trabalho. Desta forma gostaria de deixar o meu muito obrigado pelo esforço imensurável do Prof. Darci na orientação do presente trabalho.

Ao Dr. Oscar A. Z. Sotomayor pela colaboração através de informações e programas para auxiliar na validação dos resultados obtidos pela metodologia proposto no presente trabalho.

Aos meus pais - Orivaldo e Fátima, avós - Anísio e Juventina e irmãos - Hudson e Robson, pelo apoio e incentivo. Sem este apoio seria impensável poder finalizar este trabalho tendo em vista alguns percalços ocorridos no nosso dia-a-dia.

A minha noiva Gislaine pelo apoio e paciência durante o desenvolvimento deste trabalho. Sem dúvida alguma, este apoio foi, também, imprescindível para que eu pudesse ir até o final do mestrado.

À CHEMTECH e colegas (amigos) pelo apoio para a realização do presente trabalho.

Aos colegas do bloco 21 e da secretaria de pós-graduação do departamento de Engenharia Química da Universidade de São Paulo.

Finalmente, a todos que, direta ou indiretamente, me auxiliaram na execução deste trabalho. 
"Bom mesmo é ir à luta com determinação, abraçar a vida e viver com paixão, perder com classe e viver com ousadia. Pois o triunfo pertence a quem se atreve, e a vida é muito bela para ser insignificante."

Charles Chaplin 


\section{RESUMO}

Durante a operação de um controlador MPC, a planta pode ir para outro ponto de operação principalmente pela decisão operacional ou pela presença de perturbações

medidas/não-medidas. Assim, o modelo do controlador deve ser adaptado para a nova condição de operação favorecendo o controle sob as novas condições. Desta forma, as condições ótimas de controle podem ser alcançadas com a maior quantidade de modelos identificados e com um controlador adaptativo que seja capaz de selecionar o melhor modelo.

Neste trabalho é apresentada uma metodologia de controle adaptativo com identificação "on-line" do melhor modelo o qual pertence a um conjunto previamente levantado.

A metodologia proposta considera um controlador em duas camadas e a excitação do processo através de um sinal GBN na camada de otimização com o controlador em malha fechada. Está sendo considerada a validação deste controlador adaptativo através da comparação dos resultados com duas diferentes técnicas - Controlador MMPC e Identificação ARX, para a comprovação dos bons resultados desta metodologia.

Palavras-chave: Identificação em Malha Fechada. Controle MultiModelos. Controle Adaptativo de Processos. Controle Preditivo. 


\begin{abstract}
During the operation of a MPC, the plant can change the operation point mainly due to management decision or due to the presence of measured or unmeasured disturbances. Thus, the model of the controller must be adapted to improve the control in the new operation conditions. In such a way, a better control policy can be achieved if a large number of models are identified at the possible operation points and it is available an adaptive controller that is capable of selecting the best model.

In this work is presented a methodology of adaptive control with on-line identification of the most adequate model which belongs to a set of models previously obtained.

The proposed methodology considers a two-layer controller and process excitation by a GBN signal in the LP optimization layer with the controller in closed loop mode. It is also presented the adaptive controller validation by comparing the proposed approach with two different techniques - MMPC and ARX Identification, to confirm the good results with this new methodology to the adaptive controller.
\end{abstract}

Keywords: Close loop identification. Multi-Model Control. Process Adaptive Control. Model Predictive Control. 


\section{LISTA DE FIGURAS}

Figura 2-1 - Algoritmo de controle com adaptação programada e on-line .................28

Figura 2-2 - Exemplo de Excitação Externa em Malha Fechada .................................32

Figura 3-1 - Arquitetura duas camadas - Controlador MPC. …..............................34

Figura 3-2 Comportamento típico de um controlador preditivo.................................37

Figura 4-1 - P\&I para a coluna depropanizadora (Ballin, 2008)..............................51

Figura 4-2 - Pontos de operação da depropanizadora (Porfírio, 2001) ……..............53

Figura 6-1 - Simulação - Planta: Modelo 1 x Controlador: Modelo FD .....................65

Figura 6-2 - Resposta ao Degrau - Modelos Porfírio (2001)..................................67

Figura 6-3 - Resposta característica para perturbação GBN ...................................69

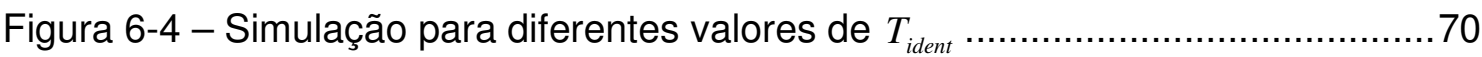

Figura 6-5 -Planta: Modelo 1 x Controlador: Modelo Combinado .............................75

Figura 6-6 -Planta: Modelo $2 \times$ Controlador: Modelo Combinado ................................76

Figura 6-7 - Planta: Modelo 3 x Controlador: Modelo Combinado ............................77

Figura 6-8 - Planta: Modelo 4 x Controlador: Modelo Combinado ...........................78

Figura 6-9 - Planta: Modelo 5 x Controlador: Modelo Combinado ...............................8

Figura 6-10 - Planta: Modelos 1 a 5 x Controlador: Modelo Combinado ...................82

Figura 6-11 - Perfil de alteração dos modelos na planta ……………………........ 82

Figura 6-12 - Degrau - Planta: Modelo 1 x Controlador: Combinação entre os

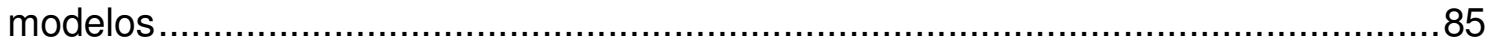

Figura 6-13 - Degrau - Planta: Modelo 2 x Controlador: Combinação entre os modelos

Figura 6-14 - Degrau - Planta: Modelo 3 x Controlador: Combinação entre os modelos.

Figura 6-15 - Degrau - Planta: Modelo 4 x Controlador: Combinação entre os modelos.

Figura 6-16 - Degrau - Planta: Modelo 4 x Controlador: Combinação entre os modelos para o pior caso.

Figura 6-17 - Degrau - Planta: Modelo 5 x Controlador: Combinação entre os modelos.

Figura 6-18 - Resposta ao Degrau para y11 e y12 respectivamente .......................93

Figura 6-19 - Resposta ao Degrau para y21 e y22 respectivamente ........................94 


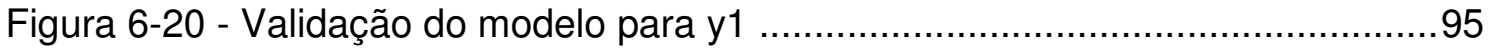

Figura 6-21 - Validação do Modelo para y2 ……………...................................95

Figura 6-22 - Resposta ao Degrau para y11 e y12 respectivamente .......................97

Figura 6-23 - Resposta ao Degrau para y21 e y22 respectivamente ........................97

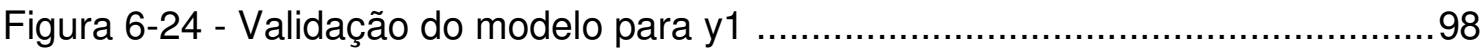

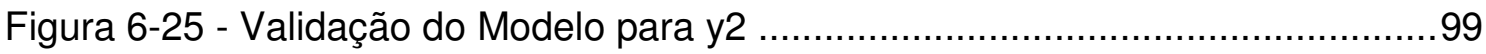

Figura 6-26 - Resposta ao Degrau para y11 e y12 respectivamente ......................100

Figura 6-27 - Resposta ao Degrau para y21 e y22 respectivamente ......................101

Figura 6-28 - Validação do modelo para y1 ……………..................................102

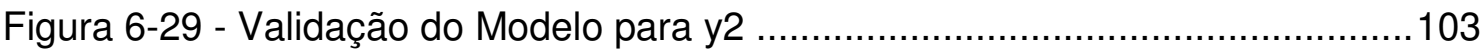

Figura 6-30 - Resposta ao Degrau para y11 e y12 respectivamente .......................104

Figura 6-31 - Resposta ao Degrau para y21 e y22 respectivamente .......................104

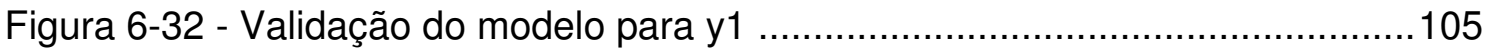

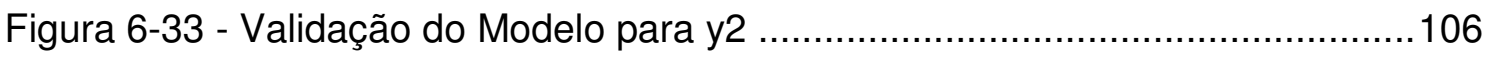

Figura 6-34 - Resposta ao Degrau para y11 e y12 respectivamente .......................107

Figura 6-35 - Resposta ao Degrau para y21 e y22 respectivamente .......................108

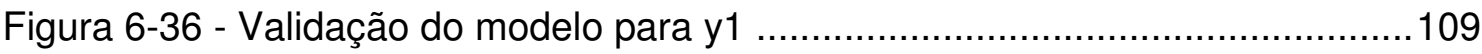

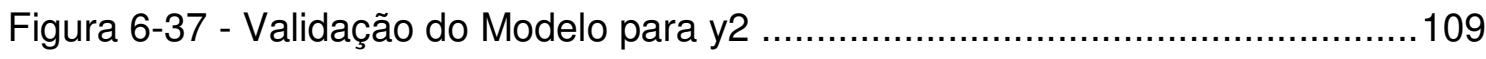

Figura 6-38 - Resposta ao Degrau para y11 e y12 respectivamente .......................111

Figura 6-39 - Resposta ao Degrau para y21 e y22 respectivamente .......................111

Figura 6-40 - Validação do modelo para y1 …………….................................112

Figura 6-41 - Validação do Modelo para y2 ………..............................................113 


\section{LISTA DE TABELAS}

Tabela 6-1 - Sintonia do Controlador - Camada dinâmica ........................................64

Tabela 6-2 - Sintonia do Otimizador - Camada Estática ..........................................65

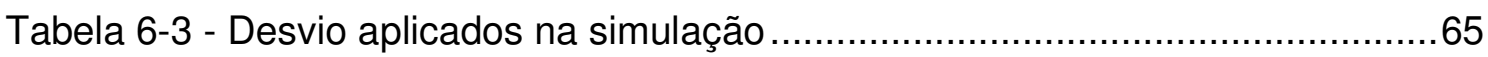

Tabela 6-4 - Parâmetros para definição do sinal GBN ..........................................68

Tabela 6-5 - Coeficientes $\lambda_{i}$ obtidos pelo Teste 1..............................................71

Tabela 6-6 - Coeficientes $\lambda_{i}$ obtidos pelo Teste 2.............................................72

Tabela 6-7 - Tempo de Experimento por Conner e Seborg (2004) ...........................73

Tabela 6-8 - Resultado da combinação entre os modelos ……………………......75

Tabela 6-9 - Resultado da combinação entre os modelos .......................................76

Tabela 6-10 - Resultado da combinação entre os modelos ……………………......78

Tabela 6-11 - Resultado da combinação entre os modelos ......................................79

Tabela 6-12 - Resultado da combinação entre os modelos ………………….........80

Tabela 6-13 - Modelo do Controlador com Planta variando do Modelo 1 a 5 ............83

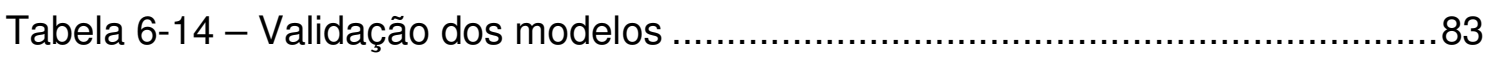

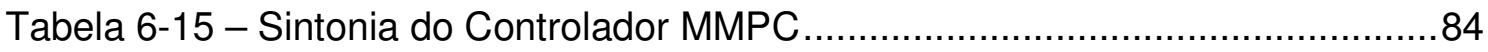

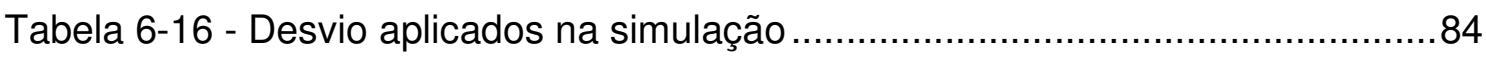

Tabela 6-17 - Modelo identificado - Planta: Modelo 1 ...........................................93

Tabela 6-18 - Modelo identificado - Case 2 ………..........................................96

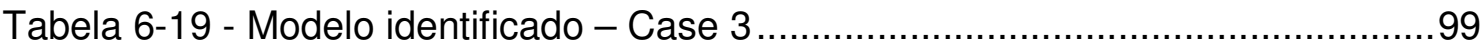

Tabela 6-20 - Modelo identificado - Case 4 .......................................................103

Tabela 6-21 - Modelo identificado - Case 5 .......................................................106

Tabela 6-22 - Modelo identificado - Case 6 .....................................................110 


\section{LISTA DE ABREVIATURAS E SIGLAS}

ADMC

ARX

ARMAX

AIC

$\mathrm{C}_{3}^{-}$

$\mathrm{C}_{4}{ }^{+}$

$\mathrm{CV}_{1}$

$\mathrm{CV}_{2}$

$\mathrm{CV}_{3}$

CONTSID

DMC

FCC

FC

FIR

FO

GBN

GLP

HYSYS $^{\text {TM }}$

ISE

LDMC

LC

LMI

LP

MPC

Matlab ${ }^{\mathrm{TM}}$

MMPC

$\mathrm{MPHC}$

$\mathrm{MV}_{1}$

$\mathrm{MV}_{2}$

$\mathrm{MV}_{3}$

$\mathrm{MV}_{4}$

OE

PID
Adaptive Model Controller

Auto-Regressive eXogenous

Autoregressive Moving Average eXogenous

Akaike Information Criterion

\% propano e componentes com pesos moleculares mais baixos

$\%$ de iso-butano e componentes de maior peso molecular

Variável controlada 1

Variável controlada 2

Variável controlada 3

Contínuos-Time System Identification

Dynamic Matrix Control

Craqueamento Catalítico Fluído

Controlador de Vazão

Finite impulse response

Função Objetivo

Generalized Binary Noise

Gás Liquefeito de Petróleo

Software de simulação de processo da empresa Hyprotech Inc Integral do erro Quadrático

Linear Dynamic Matrix Control

Controlador de Nível

Linear Matrix Inequality

Programação Linear

Model Predictive Control

Software Matemático da empresa Mathworks Inc

Multi-Model Predictive Control

Model Predictive Heuristic Control

Variável Manipulada 1

Variável Manipulada 2

Variável Manipulada 3

Variável Manipulada 4

Output Error

Controlador Proporcional Integral e Derivativo 


$\begin{array}{ll}\text { PV } & \text { Variável de Processo } \\ \text { P\#24 }_{\text {\#EM }} & \text { Pressão no estágio 24 } \\ \text { PRBS } & \text { Prediction Error Method } \\ \text { PC } & \text { Pseudo-Random Binary Sequence } \\ \text { QDMC } & \text { Controlador de Pressão } \\ \text { QP } & \text { Quadratic Dynamic Matrix Control } \\ \text { RPBC } & \text { Programação Quadrática } \\ \text { REPLAN } & \text { Refinaria Presidente Bernardes de Cubatão } \\ \text { REFAP } & \text { Refinaria de Paulínia } \\ \text { REVAP } & \text { Refinaria Alberto Pasqualini } \\ \text { RSMPC } & \text { Refinaria do Vale do Paraíba } \\ \text { ROSSMPC } & \text { Robust Stable MPC } \\ \text { STC } & \text { Reduced Order State-Space MPC } \\ \text { TC } & \text { Self-Tuning Controller } \\ \text { TGOP }_{\text {GOP }} & \text { Controlador de Temperatura } \\ T_{\text {TOPO }} & \text { Temperatura do gasóleo pesado } \\ \text { UFCC } & \text { Temperatura do topo da coluna } \\ \text { USP } & \text { Unidade de Craqueamento Catalítico Fluído } \\ V_{\text {GOP }} & \text { Universidade de São Paulo } \\ V_{\text {CARGA }} & \text { Vazão de fluido de Aquecimento } \\ V_{\text {PROPANO }} & \text { Vazão de Carga } \\ V_{F R} & \text { Vazão de Propano } \\ & \text { Vazão de Refluxo de Topo }\end{array}$




\section{LISTA DE SÍMBOLOS}

$a_{1}, a_{2} \quad$ Coeficiente do denominador do polinômio do modelo do processo

$b_{0}, b_{1} \quad$ Coeficiente do numerador do polinômio do modelo do processo

A', B' e C Matrizes dos modelos em variáveis de estado

$C_{0} \quad$ Matriz dos coeficientes da expansão da resposta ao degrau quando $n a=0$

$C_{d} \quad$ Matriz dos coeficientes da expansão da resposta ao degrau quando $n a \neq 0$

$d \quad$ Desvio entre $\hat{y}(k+1 / k)$ e $y(k+1)$

$\tilde{d} \quad$ Vetor com os desvios d

D' Matriz dos coeficientes exponencial da resposta ao degrau desconsiderando o tempo morto

$\Delta u_{\max } \quad$ Variação máxima da entrada

$e^{\prime}$

Vetor de erros antes de implementar $u(k)$

Vetor de erros após implementar $u(k)$

Vetor de erros entre $\hat{y}(k-v+1 / k)$ e $y(k-v+1)$ com $v$ variando de 1 a $T_{\text {ident }}$

$E T_{S W}^{*} \quad$ Tempo médio entre duas alterações consecutivas no GBN

$G_{i, j}(s) \quad$ Função transferência no domínio de $s$

$i \quad$ Índice da variável controlada

$I_{n y} \quad$ Matriz identidade com dimensão $n y$

j I Índice da variável manipulada

$J \quad$ Função objetivo do controlador preditivo

$\mathrm{k}$ tempo discreto no instante definido em $\mathrm{k}$

$\mathrm{K} \quad$ Ganho do processo no regime permanente

$\mathrm{K}_{\mathrm{i}} \quad$ Ganho do processo no regime permanente para o modelo $\mathrm{z}$

$L \quad$ Número de modelos possíveis do processo

$M \quad$ matriz de translação

$M^{\prime} \quad$ matriz de translação para modelo com número de estados reduzidos

n Instantes de amostragem futuros

ny Número total de variáveis controladas

nu Número total de variáveis manipuladas 


\begin{tabular}{|c|c|}
\hline nd & Número total de variáveis de distúrbios medidos \\
\hline$n p$ & Horizonte de predição. \\
\hline$n c$ & Horizonte de controle \\
\hline$n r$ & Horizonte de predição com número de estados reduzidos \\
\hline$n h$ & Horizonte de estabilização do modelo \\
\hline$N, \bar{N}$ & Matriz formada por matriz identidade $I_{n c}$ \\
\hline$n a$ & Número de pólos do modelo do processo \\
\hline$n b$ & Número de zeros do modelo do processo \\
\hline$N_{u}$ & Matriz de propagação da restrição de movimento \\
\hline$p$ e $\bar{p}$ & Estados definidos após o horizonte $n_{r}$ \\
\hline$W_{1}, W_{2}$ & Vetores Peso \\
\hline$Q$ & Matriz de pesos de importância das controladas \\
\hline$q_{i}$ & Peso de importância da variável controlada i \\
\hline$r_{i, j, g}$ & Raízes do denominador do polinômio do modelo do processo \\
\hline$R_{T}^{2}$ & Parâmetro estatístico para representar o modelo identificado \\
\hline$R$ & Matriz de pesos para os movimentos das manipuladas \\
\hline$r_{j}$ & Peso para o movimento da variável manipulada j \\
\hline$R u$ & $\begin{array}{l}\text { Matriz de pesos para conduzir as variáveis manipuladas aos seus } \\
\text { targets }\end{array}$ \\
\hline$r_{u, j}$ & Peso para conduzir a variável manipulada j ao seu respectivo target \\
\hline$S_{i}$ & $\begin{array}{l}\text { Matriz com os coeficientes da resposta ao degrau para a última ação } \\
\text { de controle definida em } \Delta u(k)\end{array}$ \\
\hline$S_{m n}$ & Matriz dinâmica com os coeficientes da resposta ao degrau \\
\hline$T_{\text {ident }}$ & Tempo de identificação para determinar os coeficientes $\lambda_{i}$ \\
\hline$T_{\text {Teste }}$ & Tempo do experimento \\
\hline$T$ & Período de amostragem \\
\hline$u_{-} 0$ & Condição inicial para as variáveis manipuladas \\
\hline$u_{s s}$ & Targets para as variáveis manipuladas \\
\hline$u_{\min }, u_{\max }$ & Limites mínimos e máximos para as variáveis manipuladas \\
\hline$u(k-1)$ & Variável manipulada em $k-1$ \\
\hline$u^{\prime}$ & Vetor de variáveis manipuladas para $\mathrm{k}-1$ \\
\hline
\end{tabular}




\begin{tabular}{|c|c|}
\hline$u_{d}$ & Vetor de desvio entre $u^{\prime}$ e $u_{s s}$ \\
\hline$x(k)$ & Vetor de estados calculados em $\mathrm{k}$ \\
\hline$x(k+1)$ & Vetor de estados calculados em $\mathrm{k}+1$ \\
\hline$W_{e x c}$ & Seqüência binária pseudo -randômica \\
\hline$y_{-} 0$ & Condição inicial para as variáveis controladas \\
\hline$y^{S P}$ & Vetor de set-point da predição \\
\hline$y_{s s}$ & Targets para as variáveis controladas \\
\hline$y_{\text {min }}, y_{\max }$ & Limites mínimos e máximos para as variáveis controladas \\
\hline $\bar{y}$ & Forma vetorial das predições após a aplicação de $\Delta u$ \\
\hline$\hat{y}$ & Forma vetorial das predições em $\mathrm{k}$ \\
\hline$\hat{y}^{C}$ & Forma vetorial das predições corrigidas em $\mathrm{k}$ \\
\hline$y^{d}$ & Forma vetorial das predições antes da aplicação de $\Delta u$ \\
\hline$\tilde{y}(k+n / k)$ & $\begin{array}{l}\text { Vetor das predições calculadas pela combinação entre os modelos em } \\
k \text { para todos os instantes } k+n \text { instantes futuros }\end{array}$ \\
\hline$\hat{y}(k+n / k)$ & $\begin{array}{l}\text { Vetor das predições calculadas em } k \text { para todos os instantes } k+n \\
\text { instantes futuros }\end{array}$ \\
\hline$y(k+n / k)$ & $\begin{array}{l}\text { Vetor das variáveis controladas calculadas em } k \text { para todos os } \\
\text { instantes de predição } k+n\end{array}$ \\
\hline$y(k+1)$ & Leitura da variável controlada obtida na planta \\
\hline
\end{tabular}

Símbolos Gregos

$\begin{array}{ll}\Delta & \text { Variação de uma variável } \\ \lambda_{z} & \text { Coeficiente de participação do modelo z no controlador } \\ \theta & \text { Tempo morto } \\ \gamma & \text { Parâmetro de otimização no MMPC } \\ \Phi & \text { Função objetivo do algoritmo QDMC } \\ \Psi & \begin{array}{l}\text { Matriz dos coeficientes exponenciais da resposta ao degrau com tempo } \\ \text { morto }\end{array} \\ \Phi_{i} & \text { Coeficiente exponencial da resposta ao degrau com tempo morto } \\ \delta_{y} & \text { Variáveis de relaxamento das saídas } \\ v & \text { Contador }\end{array}$




\section{SUMÁRIO}

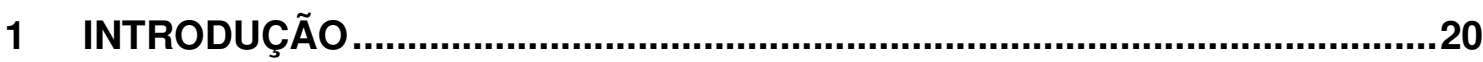

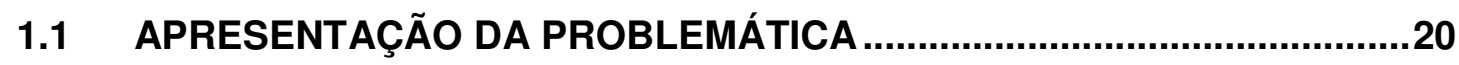

1.2 OBJETIVO

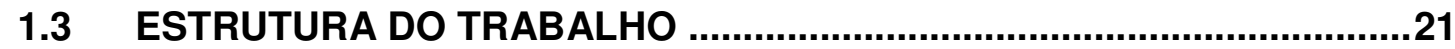

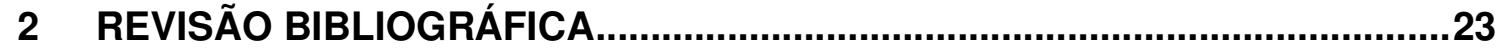

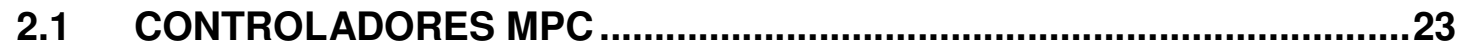

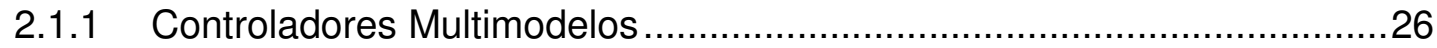

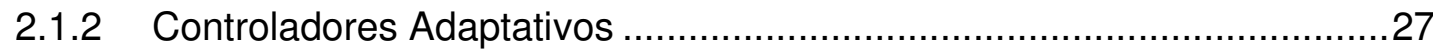

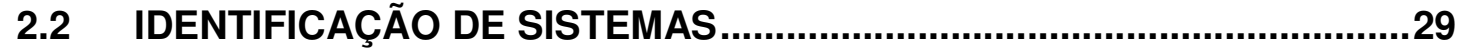

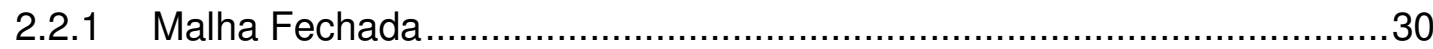

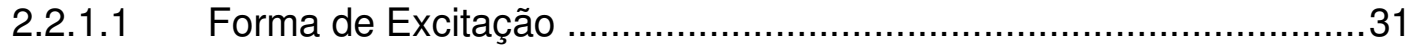

2.2.1.1.1 Excitação externa......................................................................

2.2.1.1.2 Excitação interna....................................................................

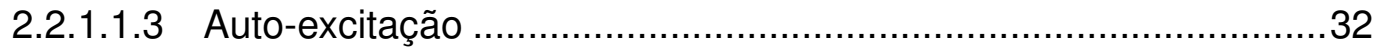

2.2.1.2 Apresentação dos Dados ...............................................................

2.2.1.2.1 Identificação Direta ..................................................................

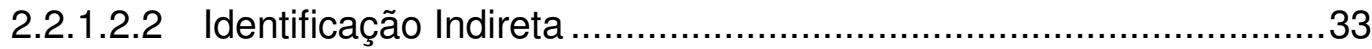

2.2.1.2.3 Identificação conjunta de entrada e saída......................................33

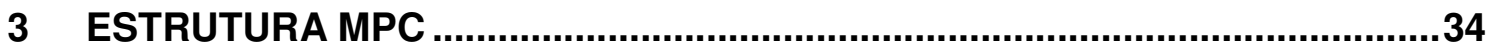

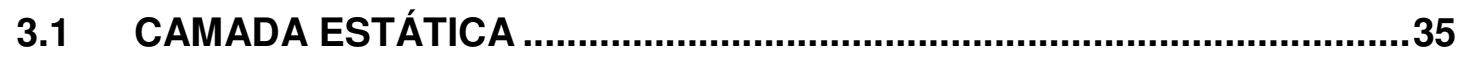

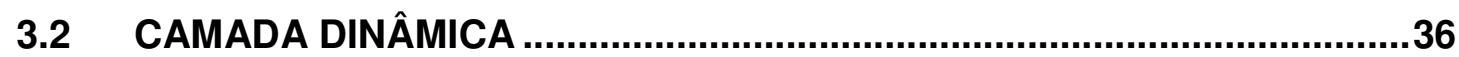

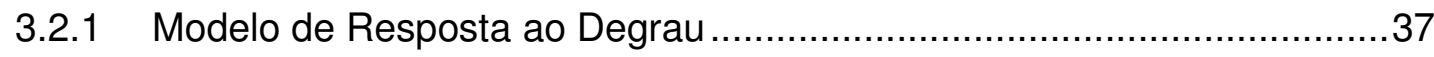

3.2.1.1 Correção do Modelo de Predição …………………………............39

3.2.2 Modelo de Resposta ao Degrau com Ordem Reduzida ...........................39

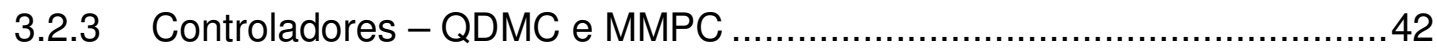

3.2.3.1 Representação do erro nas predições ........................................... 42

3.2.3.2 QDMC com Targets para as entradas............................................43

3.2.3.3 MMPC para Sistemas com Vários Modelos .....................................48

3.2.4 Estratégia de Controle por Faixa …….................................................5

4 METODOLOGIA PROPOSTA PARA O MPC ADAPTATIVO ...........................51

4.1 EXEMPLO DE SISTEMA - COLUNA DEPROPANIZADORA ....................51

4.1.1 Modelos do Processo e Matriz de Resposta ao Degrau..........................52

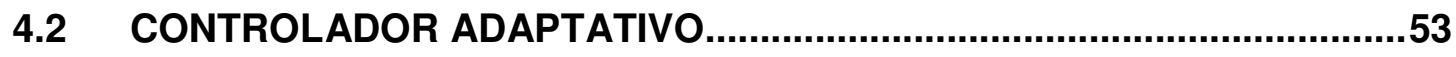

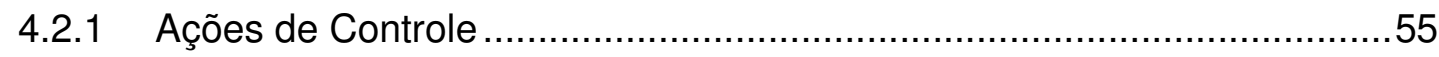




\subsection{EXCITAÇÃO PARA A IDENTIFICAÇÃO DE MODELOS PARA O CONTROLADOR ADAPTATIVO}

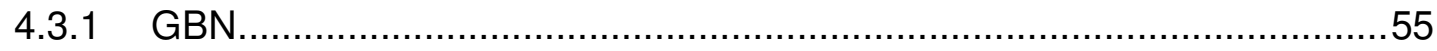

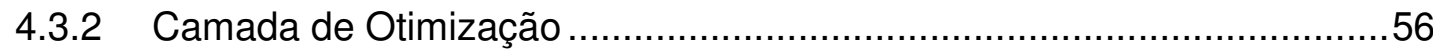

4.4 CARACTERIZAÇÃO DA EXCITAÇÃO PERSISTENTE ….........................57

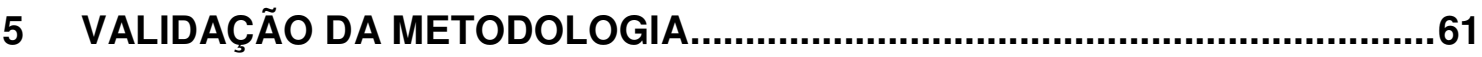

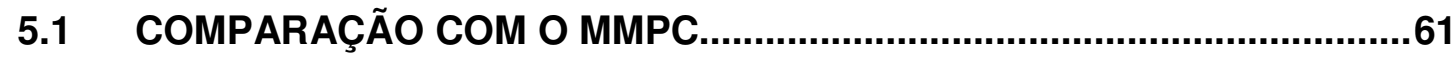

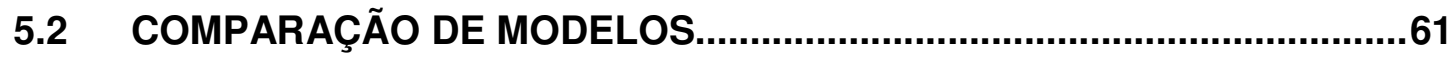

6 RESULTADOS E DISCUSSÃO

6.1 CASO REAL - COLUNA DEPROPANIZADORA …...................................64

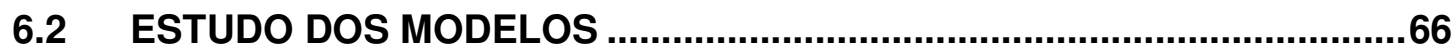

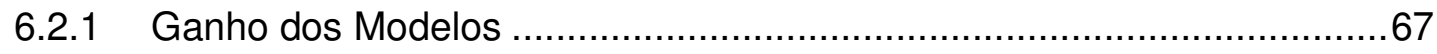

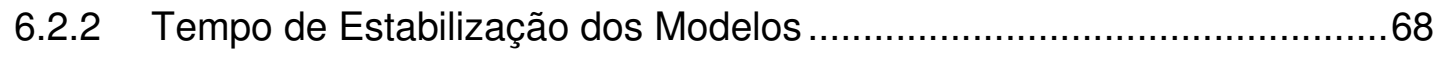

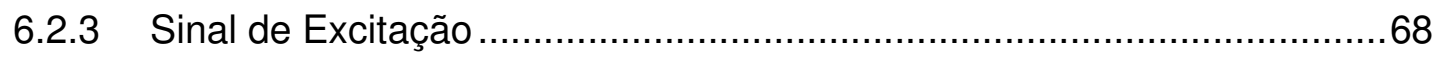

6.3 ANÁLISE PARÂMETROS DO CONTROLADOR AQDMC .........................69

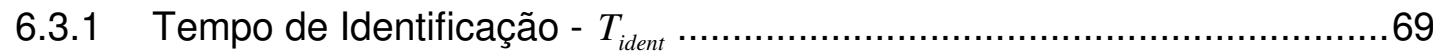

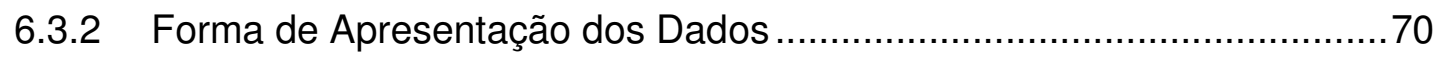

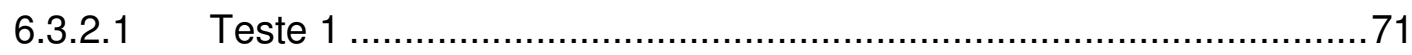

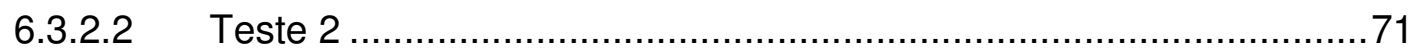

6.3.2.3 Análise Geral para a Forma de Apresentação dos Dados ................72

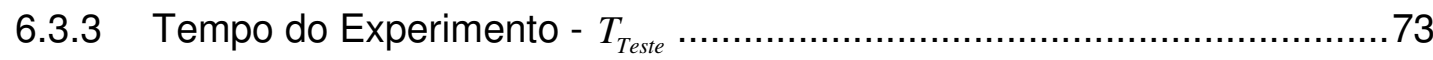

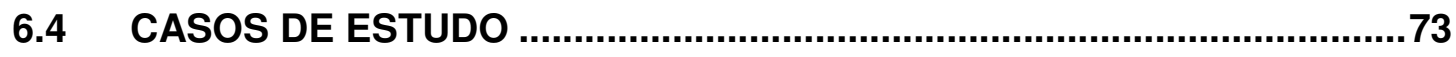

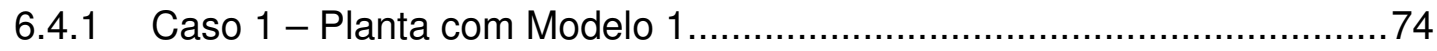

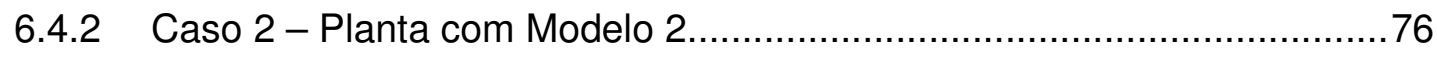

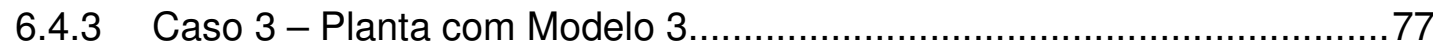

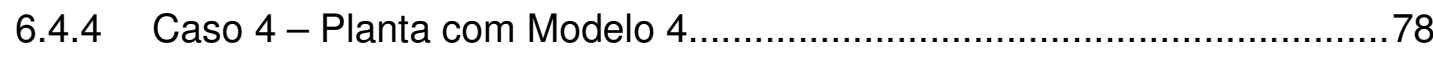

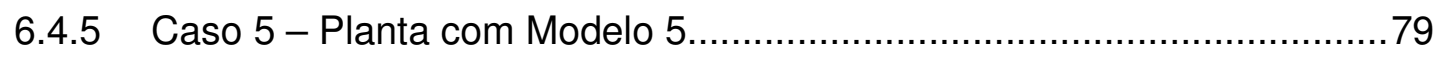

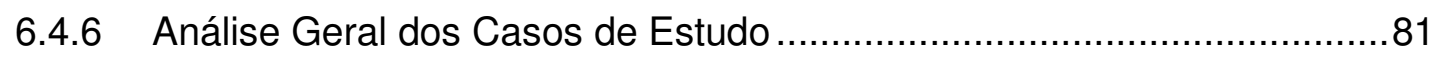

6.5 PLANTA VARIANDO ENTRE OS MODELOS 1 A 5...............................

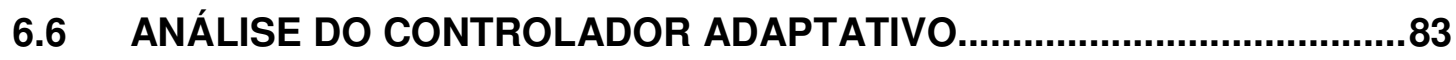

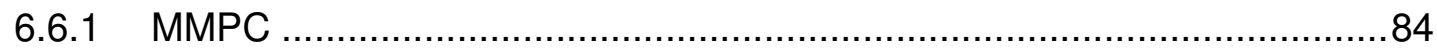

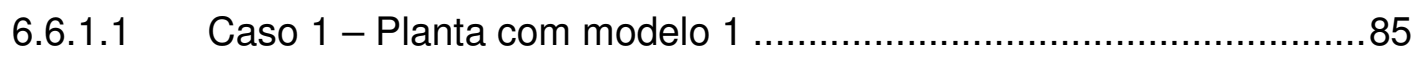

6.6.1.2 Caso 2 - Planta com modelo 2 .................................................. 85

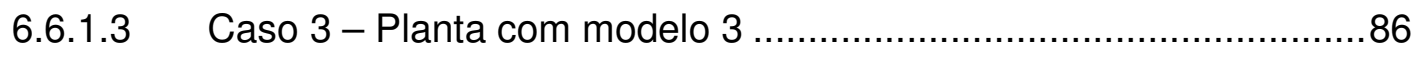

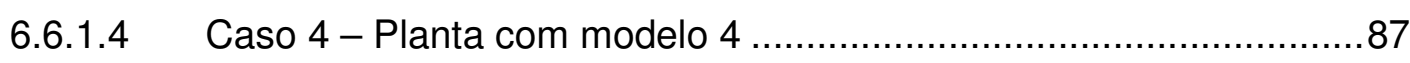


6.6.1.5 Caso 5 - Planta com modelo 4 ............................................. 89

6.6.1.6 Caso 6 - Planta com modelo 5 ............................................ 90

6.6.1.7 Análise Geral..........................................................................

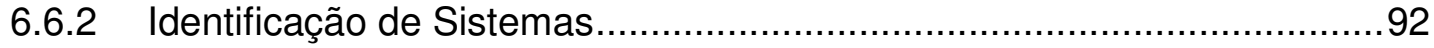

6.6.2.1 Caso 1 - Planta com Modelo 1 ....................................................92

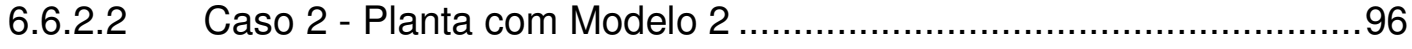

6.6.2.3 Caso 3 - Planta com Modelo 3 …................................................99

6.6.2.4 Caso 4 - Planta com Modelo 4 .................................................103

6.6.2.5 Caso 5 - Planta com Modelo 4 ................................................106

6.6.2.6 Caso 6 - Planta com Modelo 5 ................................................110

6.6.2.7 Análise dos resultados dos métodos de identificação ...................113

7 CONCLUSÕES............................................................................................114

8 CONTINUIDADE DO TRABALHO .........................................................116

REFERÊNCIAS BIBLIOGRÁFICAS ................................................................117

ANEXO A - MODELOS COLUNA DEPROPANIZADORA ..................................122 


\section{INTRODUÇÃO}

Neste capítulo são levantadas informações relativas à problemática a ser analisada neste trabalho conforme se verifica na seção 1.1. Enquanto que os objetivos e a organização do presente trabalho estão apresentados nas seções 1.2 e 1.3, respectivamente.

\subsection{Apresentação da problemática}

Controladores preditivos, em específico a classe dos MPCs, são usados intensamente na indústria de processos. Estes controladores são caracterizados pela propriedade de manter as saídas do processo sob controle através de um modelo que relaciona as entradas as suas saídas e pela inclusão de restrições operacionais para as variáveis manipuladas e controladas. Além disso, existe a inclusão de objetivos econômicos que devem ser respeitados na solução do problema de otimização para a determinação das ações de controle.

Os processos, na sua grande maioria, possuem mais de um ponto de operação, consequentemente, mais de um modelo. Assim, surge a necessidade de algoritmos de controle que considerem estas alterações de ponto de operação e que permita identificar o melhor modelo e a partir daí determinar as ações de controle baseada nas restrições operacionais e função objetivo.

Kanellakopoulos et al.(1991) considerada a utilização de controladores com mais de um modelo, simultaneamente, para controle preditivo onde as ações de controle são obtidas pelo modelo que mais se aproxima da planta.

Considerando a necessidade de se controlar uma coluna de destilação industrial com mais do que um ponto de operação, Porfírio (2001) propõe um novo algoritmo de controle denominado MMPC onde as ações de controle se baseiam em um problema de otimização mim-max onde as ações de controle são definidas pelo pior modelo entre os $L$ modelos identificados. 
A aplicação de controladores multimodelos desponta como uma técnica com real importância para os processos não-lineares onde os diferentes pontos de operação alteram, significamente, o modelo linear do processo.

\subsection{Objetivo}

O objetivo principal deste trabalho é desenvolver uma metodologia alternativa para a atualização/adaptação do modelo usado pelo MPC às condições operacionais vigentes através de um controlador MPC adaptativo. Para tanto, serão utilizados como referência, os estudos do controlador com multimodelos lineares (MMPC) de Porfírio et al (2003) com uma nova metodologia de excitação do MPC de Sotomayor et al (2008).

A seguir são apresentados alguns objetivos complementares deste trabalho:

Analisar, através de simulação, o comportamento do controlador adaptativo proposto em relação ao controle (manter as variáveis controladas dentro da faixa de operação) e ao modelo identificado em malha fechada (qualidade dos modelos);

> Avaliar o controlador adaptativo proposto, de forma comparativa, com o controlador MMPC de Porfírio (2003) verificando estabilidade e restrições após incluir perturbação;

> Estudar o modelo do controlador adaptativo identificado, comparativamente, com modelos identificados através de modelos com a forma ARX.

\subsection{Estrutura do Trabalho}

Este trabalho está dividido em oito capítulos, incluindo a Introdução.

No capítulo 1 é apresentado a problemática e o objetivo de estudo para o presente trabalho. Na problemática é apresentada a necessidade de controladores que considerem vários modelos do processo em função dos diferentes pontos de operação. O capítulo é concluído com a apresentação do objetivo do trabalho. 
No capítulo 2 é apresentada a revisão bibliográfica para embasamento teórico do presente trabalho. Basicamente, está sendo considerado um histórico sobre os controladores MPC, levantamento da literatura sobre a utilização de controladores multimodelos, adaptativos e informações relativas a identificação de sistemas, principalmente, relativas a identificação em malha fechada.

No capítulo 3 é detalhado um controlador MPC típico em duas camadas abordando a camada de otimização estacionária (PL) e a camada dinâmica enfocando o modelo de predição em variáveis de estado, a estratégia do MMPC e o correspondente problema de otimização que gera a lei de controle.

No capítulo 4 é apresentado o MPC adaptativo explicitando o problema de otimização, o tipo de excitação e a caracterização da excitação persistente para a obtenção do melhor modelo. Neste capítulo é apresentado também o exemplo de sistema considerado para as simulações.

No capítulo 5 são apresentadas as metodologias para a validação do controlador adaptativo proposto, considerando a comparação com o controlador MMPC de Porfírio et. al. (2003) e com a identificação através de modelos ARX.

No capítulo 6 são apresentadas todas as simulações e as discussões necessárias.

No capítulo 7 são apresentadas as conclusões sobre a metodologia proposta.

Finalmente, no capítulo 8 são apresentados tópicos sugeridos que podem ser considerados em trabalhos futuros. 


\section{Revisão Bibliográfica}

Faz parte da revisão bibliográfica, as seções 2.1 e 2.2, onde estão sendo discutidos tópicos relativos a Controladores MPC e Identificação em malha fechada, respectivamente.

\subsection{Controladores MPC}

Os controladores preditivos (MPCs) são uma classe de algoritmos de controle que calculam uma série de ações das variáveis manipuladas ao longo do tempo com a finalidade de otimizar o comportamento futuro da saída da planta. As primeiras aplicações foram implementadas em unidades de processo de refinarias de petróleo.

Historicamente, os MPC's foram desenvolvidos segundo a necessidade da indústria, em especial na indústria de refino de petróleo, na solução de problemas relacionados ao controle de processos. O primeiro controlador desenvolvido, na década de 70, foi o MPHC (Model Predictive Heuristic Control) proposto por Richalet et. al. (1978). Também remonta a este período o desenvolvimento do DMC (Dynamic Matrix Control) sem restrições por Cutler e Ramaker (1979).

Posteriormente ao surgimento do DMC, Prett e Gillete (1980) descreveram uma aplicação do controlador DMC sem restrições em dois níveis. O nível superior consta de um algoritmo de programação linear (LP), que resolve um problema de otimização no estado estacionário sujeito às restrições do processo. O nível inferior recebe os valores ótimos das entradas e os valores de referência das saídas, e resolve o problema através do controlador DMC sem restrições.

Apesar do aparecimento dos controladores preditivos atribuído a Richalet et al. (1978) e Cutler e Ramaker (1979), sua gênese deve ser reportada à década de 1960, quando surgiu o controle ótimo. Essencialmente, o controlador MPC é obtido pela solução de um problema de controle ótimo padrão, com a diferença de que nos controladores preditivos o problema de controle ótimo a ser resolvido deve ter um horizonte finito, de modo oposto ao horizonte infinito empregado nas abordagens do controle linear ótimo $\mathrm{H} 2$ e $\mathrm{H} \infty$. Outra diferença marcante em relação a outros controladores ótimos é que no controlador MPC o problema de controle é resolvido 
"on-line" usando a medida (ou estado) atual da planta, ao invés de uma estratégia de cálculo "off-line" (Mayne et al., 2000).

Os controladores MPHC e DMC apresentam dificuldades em incorporar as restrições nos problemas de controle. Isto deu origem a uma segunda classe de controladores preditivos. Em 1985 Morshedi et al. descreveram o algoritmo LDMC e em 1986, Garcia e Morshedi descreveram o algoritmo QDMC e apresentaram os resultados de uma aplicação em um forno de pirólise. Na abordagem do LDMC e QDMC, foi introduzido o controle por faixa, onde se controla o processo através de uma janela de operação.

Os controladores DMC, LDMC e QDMC estavam consolidados em meados da década de 80. A partir daí as pesquisas se voltaram para a análise da robustez dos mesmos, isto é, a utilização da estrutura de controle junto à planta, onde existem incertezas nas medidas e nos modelos utilizados pelo controlador. Vários trabalhos foram responsáveis por esta análise onde se pode destacar: - Rouhani e Mehra (1982); - Morari e Doyle (1986); - Morari e Zafirou (1989). Neste ponto surgiram algumas propostas de análise de estabilidade dos MPC's, entre elas uma abordagem paramétrica para o modelo do MPC's, denominada GPC (Generalized Predictive Control) proposto por Clarke et al. em 1987. Li et al. (1989) apresentam uma descrição bem detalhada do MPC em variáveis de estado, explicando as suas propriedades e apresentando melhorias em relação ao DMC, como a utilização do filtro de Kalman para modelar as perturbações não medidas.

Odloak (1995) apresentou o controlador DMC em variáveis de estado, com possibilidade de analisar o sistema de controle em malha fechada, isto é, a resposta do processo às ações do controle é equacionada juntamente com o controlador. Esta abordagem permite a verificação da estabilidade da malha fechada sem realizar as simulações do sistema. Isto possibilita o estudo da estabilidade do controlador diante dos parâmetros de sintonia e de possíveis configurações do controle.

A utilização do controlador DMC tradicional em variáveis de estado tem a dimensão do estado correspondente ao número de coeficientes da resposta ao degrau do modelo do processo. Isto leva às limitações no uso deste controlador (Lundström et 
al. 1995), como alto esforço computacional para controladores de grandes dimensões e processos com dinâmicas muito lentas. Com o objetivo de resolver este tipo de problema, foram apresentadas algumas propostas de truncamento do modelo de resposta ao degrau, entre elas (Hoyd et al., 1993), que não teve, porém muita aceitação. Outra abordagem é a compactação do controlador através da redução da ordem do modelo. Lee et al. (1994) propuseram uma redução do estado através da construção de um filtro ótimo (filtro de Kalman), cujo ganho é resolvido através da equação de Riccati. Neste último trabalho foi realizada uma conexão desta abordagem com o controlador DMC, porém os autores não conseguiram manter exatamente a forma original do DMC e QDMC.

Diante da necessidade de resolver os problemas de controle não-linear, como o controle de coluna de destilação de alta pureza que é um caso típico em unidades petroquímicas, algumas alternativas de controladores não-lineares foram propostas. Henson (1998) e Morari e Lee (1999) apresentam o problema de controle através de otimização não-linear ou mesmo a linearização do modelo no ponto de operação e o uso de um controlador linear.

Com a necessidade de se aplicar os MPC's para sistemas de grande porte (único controlador para toda uma unidade de processo) ou sistema com dinâmicas lentas, e diante das dificuldades de apresentação do controlador DMC em sua formulação original em espaço de estado e de forma reduzida, Odloak(1996) apresentou uma forma eficiente do controlador em espaço de estado de ordem reduzida. Nesta formulação, o modelo do processo é apresentado de forma analítica, através de modelos paramétricos contínuos ou discretos. Isto possibilitou a manutenção da forma original do DMC e a utilização de parâmetros contínuos, tais como, tempos mortos fracionários, a possibilidade da utilização de instantes de predição esparsos, podendo até utilizar períodos de amostragem diferentes para cada variável controlada, o que é interessante quando num mesmo controlador temos variáveis que apresentam dinâmicas lentas e outras rápidas. Esta formulação também permitiu a análise de estabilidade dos MPCs sem muito esforço computacional (Tvrzská de Gouvêia e Odloak, 1997). 


\subsubsection{Controladores Multimodelos}

A utilização de controladores multimodelos é uma alternativa interessante para resolver os problemas onde o processo é variante no tempo. Nesta abordagem resolve-se o problema de controle levando em conta todos os possíveis modelos lineares do processo. Este problema de controle pode ser resolvido através de um problema de otimização de LMI (Linear Matrix Inequality). Este enfoque foi motivado por recentes desenvolvimentos na teoria e aplicações em problemas de controle, da otimização através de LMls.

Outra abordagem para os controladores Multimodelos é a utilização de MMPCs (Multi-Model Predictive Control), sendo que o conjunto de modelos e a equação de Riccati aparecem inclusos no problema de controle. Neste caso o controlador é projetado com garantia de estabilidade (Rodrigues e Odloak, 2000).

Almeida, Rodrigues e Odloak (2000) apresentaram uma nova formulação para o controlador MPC para uma coluna debutanizadora de gasolina, o controlador MMPC bem como a aplicação do controlador RSMPC para esta coluna. A abordagem é baseada em LMl e a incerteza no modelo é considerada assumindo que o processo real pertence a um conjunto politópico convexo de possíveis plantas. O controlador tem estabilidade garantida quando uma restrição de desigualdade do tipo Lyapunov é incluída no controlador MPC.

Porfírio, Almeida e Odloak (2003) descrevem a aplicação do MMPC para uma coluna de destilação industrial que apresenta um comportamento não linear. $O$ sistema é representado por um conjunto de modelos lineares onde cada modelo corresponde a um possível ponto de operação do sistema. As ações de controle são calculadas pelo algoritmo de controle MMPC o qual se baseia em um problema de otimização mim-max onde a função custo do controlador é minimizada para o pior modelo do processo. O controlador faz uso de uma forma particular de modelo em variáveis de estado com ordem reduzida conforme apresentado em Tvrzská de Gouvêia e Odloak (1997).

Rodrigues \& Odloak (2005) desenvolveram um MPC robusto para um sistema politópico com incertezas nos modelos onde as saídas eram realimentadas e 
possuíam restrições. O propósito principal do método é que ao longo da estabilização do sistema, as entradas do sistema podem saturar tanto no estado transiente quanto no estacionário. O esforço computacional dos métodos numéricos envolvidos na implementação do controlador é substancialmente reduzido através de síntese off-line de uma série de controladores estáveis que consideram todas as possibilidades de configuração dos sistemas. O MPC on-line envolve uma simples busca pelos coeficientes de uma combinação linear dos controladores disponíveis.

\subsubsection{Controladores Adaptativos}

Os sistemas de controle adaptativos tem sido tema de pesquisa a partir da década de 60. De forma geral, estas pesquisas eram restritas a modelos lineares (Rovithakis e Christodoulou, 1994). Entretanto, os avanços na teoria de controle não-linear e, em particular, das técnicas de linearização, motivou o desenvolvimento de esquemas de controle adaptativo para plantas não-lineares (Kanellakopoulos et al., 1991).

Em estudo realizado em controladores adaptativos pode-se destacar o STC (Controlador Auto-Ajustável) de Bolzan (1991) para o controle da temperatura de um reator semi-batelada. Os resultados demonstraram que o STC se apresenta superior a controladores clássico tais como GPC e PID.

Maiti e Saraf (1995) propõem um algoritmo de controle adaptativo, denominado ADMC, onde o distúrbio é identificado automaticamente de tal forma a se realizar a identificação do processo e posteriormente a alteração do novo modelo no controlador MPC.

Muniz (2004) define os controladores adaptativos como sistemas de controle que automaticamente ajustam seus parâmetros de modo a compensar mudanças no processo ou ambiente.

Os controladores adaptativos podem ser classificados dentro de duas grandes categorias, relativas ao momento da identificação do modelo, conforme apresentado por Muniz (2004):

$>$ Programada; 
$>$ "On-line".

A forma programada de ajustes do controlador adaptativo deve ser empregada quando se pode antecipar ou mesmo medir os distúrbios no processo controlado. Assim, nesta abordagem, deve existir um conhecimento sobre as variáveis que são responsáveis pelas mudanças nas características do processo. Ao se verificar qualquer distúrbio, o modelo do processo deve ser identificado e o modelo obtido, substituído no controlador.

A abordagem on-line é caracterizada por não ter medidas dos distúrbios que possam caracterizar o modelo. Assim, uma aproximação alternativa é identificar o modelo do processo à medida que novos dados são adquiridos e, desta forma, obter as ações de controle a partir do novo modelo. Pode-se destacar o Self-Tuning Regulator como um exemplo de adaptativo "on-line", proposto em 1973 por Astrom e Wittenmark e que a partir de então tem sido utilizado em diversas aplicações industriais (Seborg et al, 1989). Modificações subseqüentes para este controlador permitiram o surgimento do Self-Tuning Controller (Muniz, 2004).

As duas formas dos controladores adaptativos - programados e "on-line", podem ser representados conforme o diagrama de blocos na Figura 2-1.

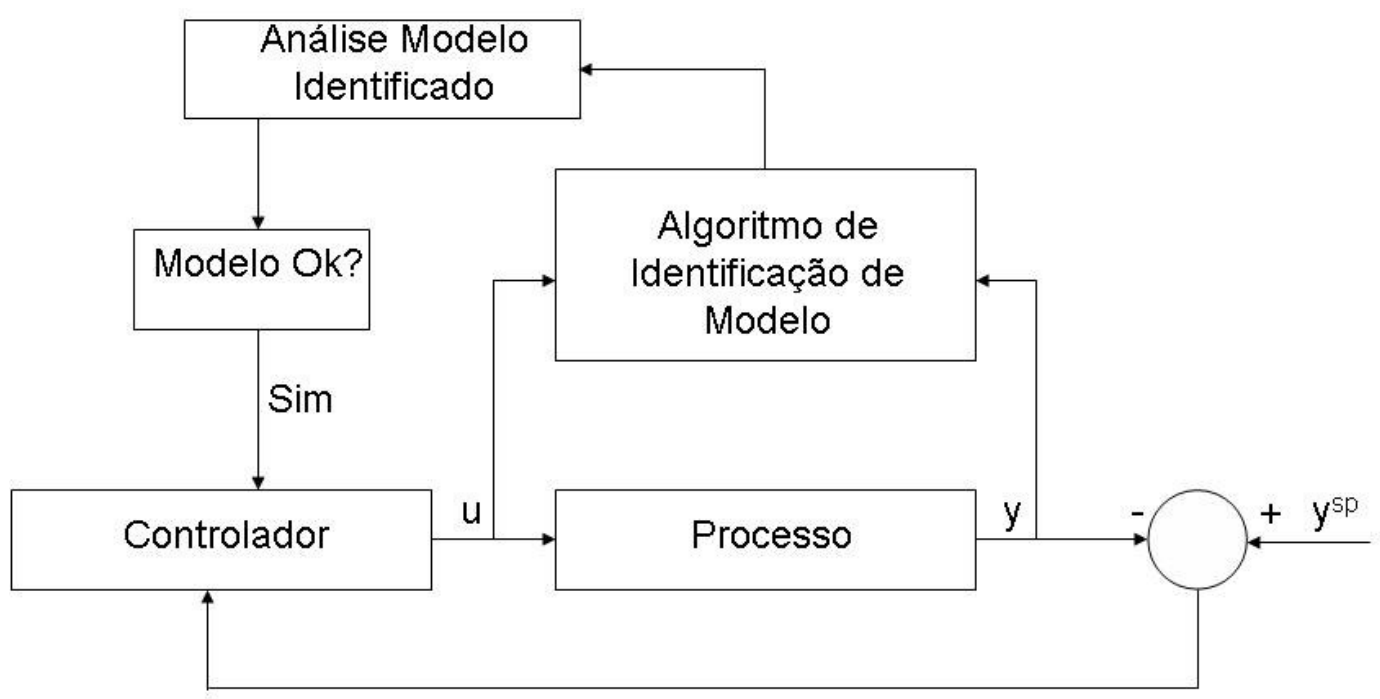

Figura 2-1 - Algoritmo de controle com adaptação programada e on-line 
Na Figura 2-1, o bloco do algoritmo de Identificação de Modelo determina os parâmetros do modelo que melhor representa o processo para o conjunto de entradas e saídas disponibilizados. Posteriormente, este modelo identificado é analisado quanto à resposta ao degrau e o porcentual de ajuste entre as saídas obtidas pelo modelo e saídas presente no conjunto de dados utilizados para a identificação. Sendo positivo o resultado nesta análise, o novo modelo é implementado no controlador.

\subsection{Identificação de Sistemas}

Nos últimos anos é evidente o desenvolvimento de técnicas de identificação as quais permitem a obtenção de modelo para controle de processos que representem com maior qualidade o processo. Estes modelos obtidos pela identificação são classificados como empíricos uma vez que utilizam dados reais obtidos ao longo de testes de excitação na planta.

Zadeh (1962, apud Garcia 2006) define a identificação como sendo a determinação, com base na entrada e na saída, de um sistema dentro de uma classe específica de sistemas, a que o sistema em teste é equivalente.

Assim, Garcia (2006) apresenta o procedimento de identificação como pode ser resumido nos itens a seguir:

1) Planejamento dos testes e coleta de dados;

2) Análise dos dados obtidos;

3) Determinação da estrutura do modelo;

4) Estimativa dos parâmetros;

5) Validação do modelo.

O Planejamento dos testes e coleta de dados é composto basicamente pela definição das variáveis de entrada, de saída, sinais de excitação do sistema, período de amostragem e, finalmente, a definição do número de instantes para serem armazenados. Para os sinais de excitação, geralmente são utilizados - PRBS, GBN, degrau, impulso, entre outros. 
Além disso, é no planejamento que se define se o experimento será executado em malha aberta ou fechada. Principalmente para processos os quais não podem ser interrompidos para a realização de testes surgem metodologias para identificação em malha fechada. Outros pontos relativos a esta abordagem estão discutidos na seção 2.2.1.

Após obter os dados deve-se considerar a etapa de análise destes dados conforme definido na seção Análise dos dados. Considera-se a utilização de filtros de primeira ordem com intuito de retirar ruídos.

Posteriormente, deve-se definir a estrutura do modelo onde as principais estruturas são - ARX, ARMAX, OE, Função transferência, entre outras.

Tendo os dados e a estrutura do modelo deve-se ajustar os parâmetros deste modelo. Os métodos numéricos de ajustes podem ser formulados como um problema de otimização, onde o melhor modelo é aquele que melhor ajusta os dados, de acordo com um dado critério. Alguns critérios que são muito utilizados são - AIC, função perda, FPE e FIT e $R_{T}^{2}$.

\subsubsection{Malha Fechada}

O interesse por esta metodologia vem crescendo na última década conforme apresentado em Landau (2001). Como exemplo, pode-se citar os trabalhos de Miranda (2005) e Sotomayor et al (2008).

Miranda (2005) propôs para um sistema controlado por um MPC, a alteração, através de um sinal PRBS, dos limites (faixas) das variáveis controladas para se verificar o efeito das manipuladas em um controlador de uma coluna de destilação atmosférica. Desta forma, foi possível obter um conjunto de dados de entrada e saída permitindo identificar um modelo na forma de um ARX que representasse o processo em questão.

Sotomayor et al. (2008), propôs para o MPC em duas camadas, uma metodologia de identificação em malha fechada onde o sinal de teste (GBN) é introduzido nos coeficientes da função objetivo da camada estática (econômica) de tal forma a perturbar o sistema e, posteriormente, identificar o modelo do processo em questão. 
Como principal característica da identificação em malha fechada tem-se o fato do processo continuar operando seguindo a especificação de produto e as restrições dos equipamentos. Desta forma, esta metodologia garante aspectos de segurança para a operação da planta.

Algumas pesquisas buscam identificar também se experimentos em malha fechada não seriam pouco informativos para a obtenção de modelos. Entretanto, segundo Ljung (1999), um sistema pode ser identificado se os dados do experimento forem suficientemente informativos e se o conjunto de modelos contiver o sistema real, independentemente, de o sistema estar em malha aberta ou malha fechada.

Garcia (2006) define que uma das condições para que o sistema seja suficientemente informativo, é que o sinal injetado na entrada do sistema deva pertencer a uma classe intitulada persistentemente excitante.

A seguir são tratados dois aspectos que devem ser analisados na identificação em malha fechada - Forma de excitação e Apresentação dos Dados.

\subsubsection{Forma de Excitação}

Sotomayor et al. (2008) definem três formas de excitação: externa, interna e autoexcitação, as quais são apresentadas a seguir.

\subsection{Excitação externa}

A excitação externa pode ser implementada nas entradas $u(t)$ e/ou nas variáveis de referências $y^{S P}$. Esta forma de excitação está apresentada na Figura 2-2. 


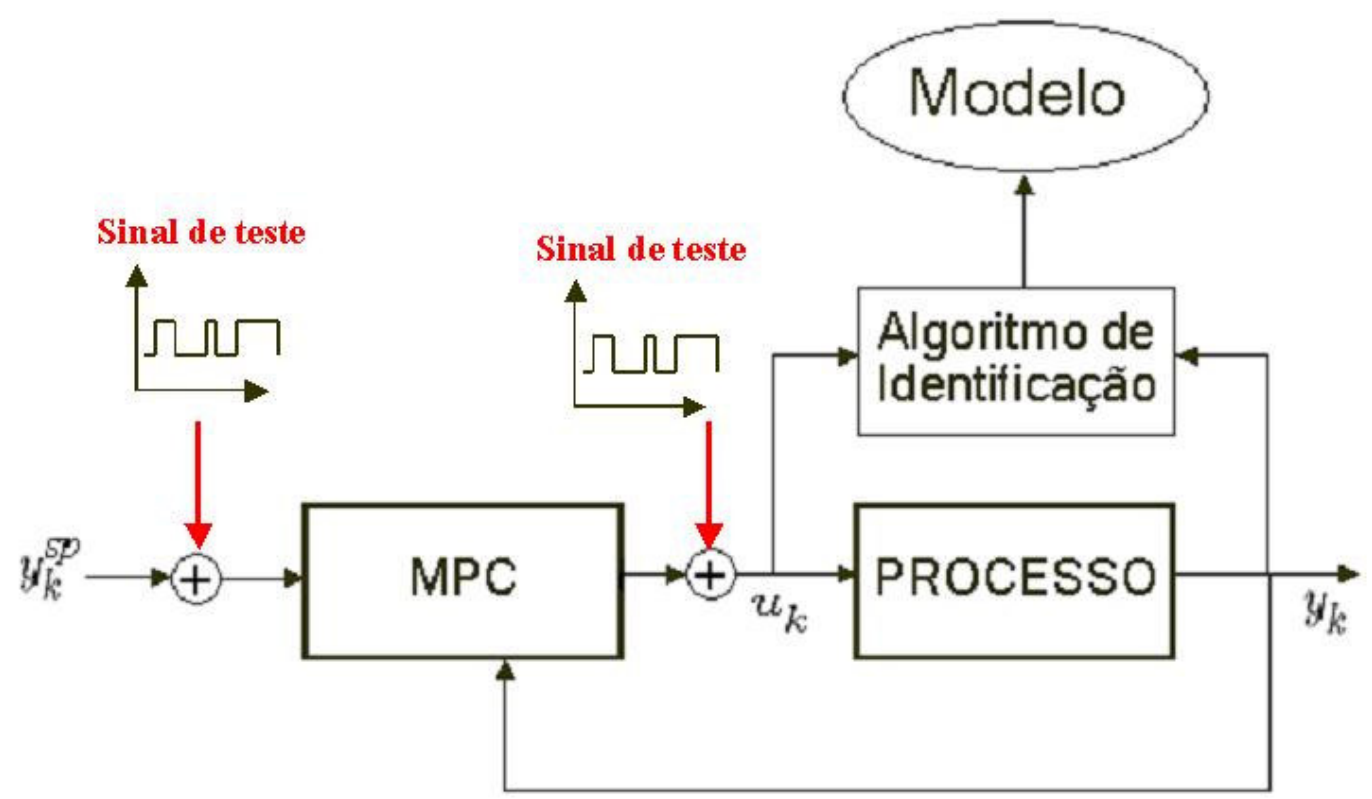

Figura 2-2 - Exemplo de Excitação Externa em Malha Fechada

Como exemplo de aplicação da excitação externa tem-se o trabalho de Miranda (2005) o qual aplicou a excitação nas restrições ativas do controlador.

É importante ressaltar que nesta abordagem não há a garantia que as restrições do processo e de especificações dos produtos serão respeitadas durante a excitação dependendo da natureza do sinal e onde o mesmo é implementado.

\subsection{Excitação interna}

Neste caso, a excitação é aplicada internamente ao controlador, por exemplo, na função objetivo fazendo com que não se tenha somente o objetivo de controle, mas também, de identificação.

\subsection{Auto-excitação}

Neste caso, propõe-se uma condição persistentemente excitante das entradas como restrição no problema de otimização, a qual pode estar ligada ou desligada conforme se tenha interesse ou não em se realizar a identificação. Exemplo desta excitação pode ser verificado em Ballin (2008). 


\subsubsection{Apresentação dos Dados}

Além da forma como é realizada a excitação tém-se também como os dados de entrada e saída são apresentados para o algoritmo de identificação para a determinação dos parâmetros do modelo. A seguir, têm-se as 3 possíveis formas conforme descrito em Garcia (2006).

\subsection{Identificação Direta}

Nesta abordagem os dados de entrada $u(t)$ e saída $y(t)$ são tratados exatamente como se eles fossem obtidos de um experimento em malha aberta, ou seja, desconsiderando a realimentação.

\subsection{Identificação Indireta}

Para esta abordagem, o modelo identificado será obtido utilizando os sinais de referência $y^{s p}$ e a saída $y(t)$. Com este sistema em malha fechada e conhecendose o controlador, pode-se determinar o modelo da planta.

\subsection{Identificação conjunta de entrada e saída}

Em alguns casos pode ser vantajoso considerar tanto a entrada quanto a saída conjuntamente, como se fossem saídas de um sistema excitado apenas por perturbações e entradas extras. Os dados da saída $y(t)$ e da entrada $u(t)$ são tratados como se fossem saídas de um sistema multivariável excitado por perturbação $v(t)$ e pela referência $y^{s p}$. 


\section{ESTRUTURA MPC}

Os controladores MPC são caracterizados por controlar um determinado processo através do cálculo de uma seqüência de ações de controle através de um problema de otimização sujeito às restrições operacionais. Com o maior conhecimento dos processos químicos e também pelo levantamento de modelos econômicos, surge a necessidade de se avaliar como restrição do MPC, os targets para a seqüência de variáveis manipuladas. Desta forma, tem-se a abordagem de controladores MPC em duas camadas conforme apresentado na Figura 3-1.

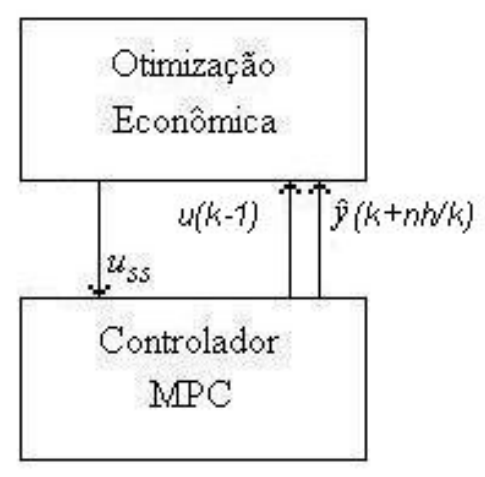

Figura 3-1 - Arquitetura duas camadas - Controlador MPC.

onde:

$u(k-1)$ - última ação de controle implementada no processo;

$n h$ - horizonte de estabilização do modelo;

$\hat{y}(k+n h / k)$ - predição da saída no estado estacionário no instante $k+n h$ calculado em $k$;

$u_{s s}$ - targets para as variáveis manipuladas na camada do MPC

Conforme apresentado na Figura 3-1, a camada superior do MPC é denominada camada estática e possui como principal característica o cálculo de otimização econômica com modelos lineares, determinando os targets das variáveis manipuladas para a camada do MPC em uma taxa de execução igual à da camada dinâmica. 
A camada inferior, também denominada, camada dinâmica, é composta pelo controlador propriamente dito onde são determinadas as ações de controle para serem implementadas no processo em uma taxa de execução definidas em minutos.

\subsection{Camada Estática}

É nesta camada que se tem a otimização econômica através da solução de um problema de programação linear (LP) ou quadrática (QP) com restrições, usando um modelo do processo em estado estacionário e informações vindas do controlador MPC, referentes ao último valor do vetor de predição das variáveis controladas $\hat{y}(k+n h / k)$, e a última variável manipulada implementada $-u(k-1)$.

No caso, a variável $\hat{y}(k+n h / k)$ definida para 0 instante de predição $k+n h$ representa um valor da variável controlada no estado estacionário mesmo sem o processo ter atingido tal condição. A partir desta informação em estado estacionário, a otimização econômica tem condições de fornecer os targets, também em estado estacionário, para as variáveis manipuladas do controlador.

Entretanto, segundo Bezerra et al. (1999), esta formulação tem a desvantagem de não otimizar a planta durante transitórios, tais como os produzidos por perturbações ou mudanças no regime operacional. Assim, para contornar eventuais problemas de viabilidade na solução da otimização, tem-se a inclusão de variáveis de relaxamento de tal forma a relaxar as restrições e permitir a obtenção de solução.

Segundo Sotomayor et al. (2008), uma vantagem destacada pela utilização da camada estática é devido a otimização econômica prover uma solução em estado estacionário que satisfaça as restrições da camada dinâmica, favorecendo assim, a estabilidade do controlador.

Assim, podemos utilizar a função objetivo (3.1), a qual representa uma LP, para o problema de otimização na camada Estática.

$$
\min _{\Delta u_{s}, \delta_{y}} \mathrm{~W}_{1}^{\mathrm{T}} \Delta u_{s s}+\left|\mathrm{W}_{2}^{\mathrm{T}} \delta_{y}\right|
$$

sujeito a: 


$$
\begin{aligned}
& \Delta u_{s s}=u_{s s}-u(k-1) \\
& y_{s s}=K \cdot \Delta u_{s s}+\hat{y}(k+n h / k) \\
& u_{\min } \leq u_{s s} \leq u_{\max } \\
& y_{\min } \leq y_{s s}+\delta_{y} \leq y_{\max }
\end{aligned}
$$

onde:

$u(k-1)$ - última ação de controle aplicado no processo

$u_{\min }, u_{\max }$ - limites das variáveis manipuladas

$K$ - matriz de ganhos do processo em regime permanente

$y_{s s}$ - predição da saída no estado estacionário

$y_{\min }, y_{\max }-$ limites das variáveis controladas

$W_{1}, W_{2}$ - vetores pesos

$\delta_{y} \quad$ - vetor de variáveis de relaxamento para as saídas do controlador.

Conforme apresentado no problema de otimização, a solução está sujeita às restrições tanto nas variáveis controladas como nas manipuladas. Para eventuais problemas de obtenção de solução para o problema de otimização, foi incluído um vetor de relaxamento representado por $\delta_{y}$ para garantir que o problema sempre tenha solução.

\subsection{Camada Dinâmica}

Uma vez definida a camada estática, a camada dinâmica utiliza os targets enviados e determina a seqüência de ações de controle através dos problemas de otimização que serão analisadas posteriormente. $\mathrm{Na}$ Figura 3-2 é apresentado o comportamento típico de um processo sob controle através de uma abordagem MPC onde se deseja ficar o mais próximo possível dos set-points das variáveis controladas. 


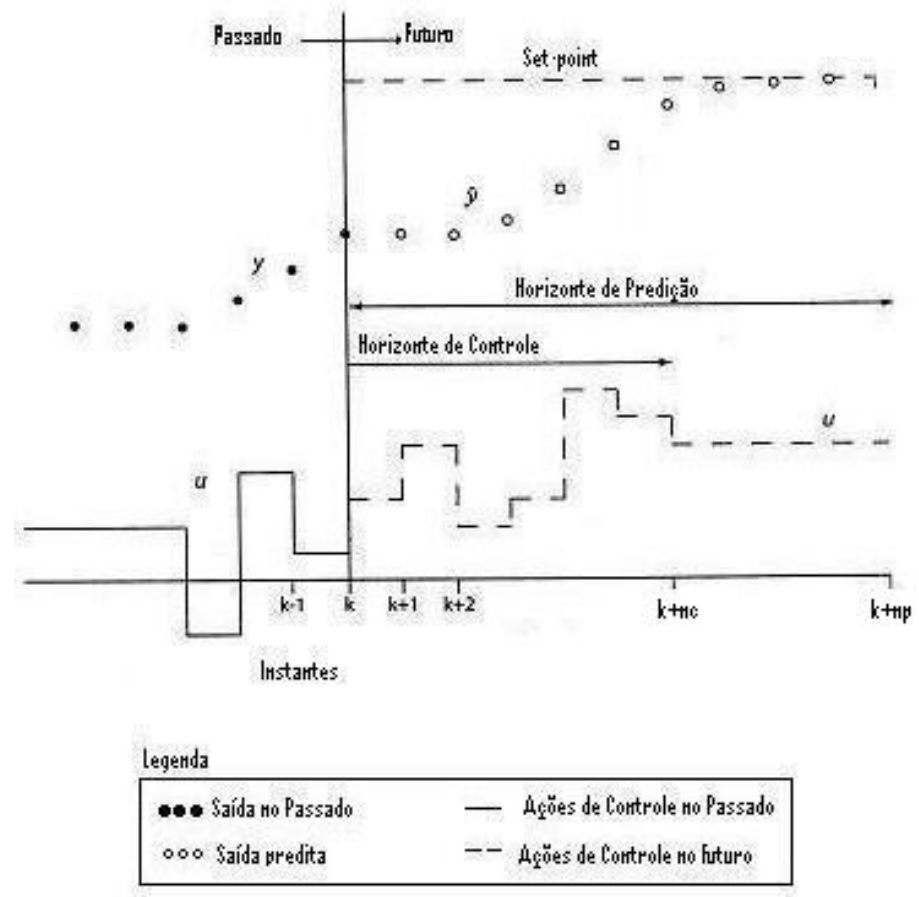

Figura 3-2 Comportamento típico de um controlador preditivo

onde:

$n c$ - horizonte de controle para o qual é determinada a seqüência de ações de controle;

$n p$ - horizonte de predição para o qual são calculadas as predições para as variáveis controladas.

A seguir serão apresentados os principais componentes que formam a camada dinâmica, começando pelos modelos de resposta ao degrau, posteriormente apresentando os principais controladores e as principais estratégias de controle.

\subsubsection{Modelo de Resposta ao Degrau}

Lee et al. (1994) formularam que, para sistemas estáveis, o modelo de resposta ao degrau pode ser representado em variáveis de estado conforme apresentado em (3.2).

$$
\begin{aligned}
& x(k+1)=M \cdot x(k)+S . \Delta u(k) \\
& y(k)=C \cdot x(k)
\end{aligned}
$$

onde: 
$M$ - matriz de translação $M=\left[\begin{array}{ccccc}0 & I_{n y} & 0 & \cdots & 0 \\ 0 & 0 & I_{n y} & \cdots & 0 \\ \vdots & \vdots & \vdots & \ddots & \vdots \\ 0 & 0 & 0 & \cdots & I_{n y} \\ 0 & 0 & 0 & \cdots & I_{n y}\end{array}\right] \quad M \in \mathfrak{R}^{(n y . n h) x(n y . n h)}$ $S-\left[\begin{array}{c}S_{1} \\ S_{2} \\ \vdots \\ S_{n c} \\ \vdots \\ S_{n p} \\ \vdots \\ S_{n h}\end{array}\right] \quad S \in \Re^{(n y . n h) x(n u)}$

$S_{i}$ - matriz correspondente aos coeficientes da resposta ao degrau no instante $i$

$n h$ - horizonte de estabilização do modelo

ny - número de variáveis controladas

$n u$ - número de variáveis manipuladas

$C=\left[\begin{array}{llll}I_{n y} & 0 & \ldots & 0\end{array}\right] \quad C \in \mathfrak{R}^{(n y) x(n y . n h)}$

$x(k)$ - é o vetor de estados com $x(k)=\left[\begin{array}{c}y(k+1 / k) \\ y(k+2 / k) \\ \vdots \\ y(k+n h / k)\end{array}\right] x(k) \in \mathfrak{R}^{(n y . n h) x(1)}$

$\Delta u(k)$ - ação de controle aplicada no instante $k \quad \Delta u(k) \in \mathfrak{R}^{(n u) x(1)}$

Observe que em M considera-se que $y(k+n h+1 / k)=y(k+n h / k)$.

Assim, a predição das variáveis controladas pode ser escrita, para os $n p$ instantes, conforme apresentado a seguir.

$\hat{y}(k+n / k)=\bar{N} \cdot x(k)$ 
onde:

$n=1$ até $n p$

$\bar{N}=\left\lfloor\begin{array}{ll}I_{n p . n y} & 0_{(n p . n y) x(n h . n y)}\end{array}\right\rfloor$

\subsubsection{Correção do Modelo de Predição}

No caso real onde um controlador MPC está controlando um processo qualquer, o modelo do controlador não representará de forma total a planta sob controle. Assim, para que a predição não se torne irreal, deve-se determinar o desvio (d) conforme apresentado em (3.3) e posteriormente aplica-lo a todo o vetor de predição $\hat{y}$.

$d=y(k+1)-\hat{y}(k+1 / k)$

onde:

$y(k+1)$ - saída lida da planta no instante $k+1-y(k+1) \in \mathfrak{R}^{(n y) x(1)}$

A correção do vetor de predição é calculada somente para o primeiro instante de predição $(k+1)$. Entretanto, o desvio $d$ deve ser propagado para todos os instantes de predição $(n p)$ de tal forma que a nova predição da resposta possa ser escrita conforme apresentado em (3.4).

$\hat{y}^{C}=\hat{y}+\tilde{d}$

onde:

$\tilde{d}=\left[\begin{array}{llll}d & d & \ldots\end{array}\right]^{T} \quad \tilde{d} \in \mathfrak{R}^{(n p) x(1)}$

$\hat{y}^{C}$ - vetor de predição corrigido - $\left[\hat{y}^{C}(k+1 / k) \hat{y}^{C}(k+2 / k) \ldots \hat{y}^{C}\left(k+n_{p} / k\right)\right]^{T}$ $\hat{y}^{C} \in \mathfrak{R}^{(n y . n p) x(1)}$

\subsubsection{Modelo de Resposta ao Degrau com Ordem Reduzida}

Segundo Porfírio et al. (2003), um processo químico pode ser geralmente representado por um modelo linear de baixa ordem. Neste caso, o modelo em espaço de estado representado anteriormente em (3.2) não é de ordem mínima uma 
vez que o número de estados é dado por ny.nu.nh. Entretanto, mesmo com o grande número de estados, a aproximação apresentada em (3.2) não traz problemas de implementação em termos de esforço computacional conforme testado em diversas aplicações industriais. Entretanto, para controladores com diversos modelos de processo se torna necessária uma forma mais econômica para representar os modelos em espaço de estado.

O modelo em espaço de estado de resposta ao degrau com ordem reduzida foi estudado por Tvrzská de Gouvêa e Odloak(1997) e Porfírio et al. (2003), posteriormente, utilizaram esta representação para a implementação do controlador MMPC conforme será visto mais adiante.

Conforme apresentado em Porfírio et al. (2003), esta nova formulação é apresentada a seguir.

$$
\begin{aligned}
& x(k+1)=A^{\prime} \cdot x(k)+B^{\prime} . \Delta u(k) \\
& y(k)=C \cdot x(k)
\end{aligned}
$$

onde:

$x(k)$ - vetor de estados de predição

$x(k)=\left[y(k / k)^{T} y(k+1 / k)^{T} \cdots y(k+n r-1 / k)^{T} \bar{p}^{T} p^{T}\right]^{T} \quad x \in \mathfrak{R}^{(n y .(n r+1)+n u . n y . n a) x(1)}$

$n a$ - ordem máxima da função transferência que representa uma saída em relação a uma entrada

$n r \quad$ é tal que $n r>n c+\max \theta_{i, j}$

$$
A^{\prime}=\left[\begin{array}{ccccccc}
0 & I_{n y} & 0 & \cdots & 0 & 0 & 0 \\
0 & 0 & I_{n y} & \cdots & 0 & 0 & 0 \\
\vdots & \vdots & \vdots & \cdots & \vdots & \vdots & \vdots \\
0 & 0 & 0 & \cdots & I_{n y} & 0 & 0 \\
0 & 0 & 0 & \cdots & 0 & I_{n y} & \Psi(n r T) \\
0 & 0 & 0 & \cdots & 0 & I_{n y} & 0 \\
0 & 0 & 0 & \cdots & 0 & 0 & D^{\prime}
\end{array}\right]
$$


$\begin{aligned} \Psi(t) & =\left[\begin{array}{cccc}\phi_{1}(t) & 0 & \cdots & 0 \\ 0 & \phi_{2}(t) & \cdots & 0 \\ \vdots & \vdots & \ddots & \vdots \\ 0 & 0 & \cdots & \phi_{n y}(t)\end{array}\right] \\ \phi_{i}(t) & =\left[e^{r_{i, 1,1}\left(t-\theta_{i, 1}\right)} \cdots e^{r_{i, 1, n a}\left(t-\theta_{i, 1}\right)} \cdots e^{r_{i, n u, 1}\left(t-\theta_{i, n u}\right)} \cdots e^{r_{i, n u, n a}\left(t-\theta_{i, n u}\right)}\right]\end{aligned}$

$r_{i, j, k}$ - k-ésimo pólo da função de transferência que relaciona $y_{i}$ com $u_{j}$

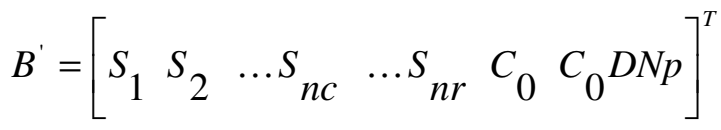

$C=\left[\begin{array}{llll}I_{n y} & 0 & \cdots & 0\end{array}\right]$

$\bar{p}$ - estado que define a predição da saída no estado estacionário $\bar{p}=\left[\bar{p}_{1} \bar{p}_{2} \cdots \bar{p}_{n y}\right]^{\mathrm{T}}$

$p$ - estado associado com os modos estáveis do sistema $p=\left[\begin{array}{llllllll}p_{1,1,1} & p_{1,1,2} & \cdots & p_{1,2, n a} & p_{1,2,1} & p_{1,2,2} & \cdots & p_{1,2, n a}\end{array} \cdots p_{n y, 1,1} p_{n y, 1,2} \cdots p_{n y, 1, n a} \cdots p_{n y, n u, 1} p_{n y, n u, 2} \cdots\right.$ $\left.p_{n y, n u, n a}\right]^{T}$

$D^{\prime}=\begin{aligned} & \operatorname{diag}\left[e^{r_{1,1,1} T} \cdots e^{r_{1,1, n a} T} e^{r_{1,2,1} T} \cdots e^{r_{1,2, n a} T} \cdots e^{r_{1, n u, 1} T} \cdots e^{r_{1, n u, n a} T} e^{r_{2,1,1} T} \cdots e^{r_{2,1, n a} T} \cdots e^{r_{n y, 1,1} T} \cdots e^{r_{n y, 1, n a} T} \cdots\right. \\ & \left.e^{r_{n y, n u, 1} T} \cdots e^{r_{n y, n u, n a} T}\right]\end{aligned}$ $N_{p}=\left[\begin{array}{llll}J_{1} J_{2} & \cdots & J_{n y}\end{array}\right]^{\mathrm{T}}$ $\left.J_{i}=\left[\begin{array}{ccccc}1 & 0 & 0 & \cdots & 0 \\ \vdots & \vdots & \vdots & \ddots & \vdots \\ 1 & 0 & 0 & \cdots & 0 \\ 0 & 1 & 0 & \cdots & 0 \\ \vdots & \vdots & \vdots & \ddots & \vdots \\ 0 & 1 & 0 & \cdots & 0 \\ \vdots & \vdots & \vdots & \ddots & \vdots \\ 0 & 0 & 0 & \cdots & 1 \\ \vdots & \vdots & \vdots & \ddots & \vdots \\ 0 & 0 & 0 & \vdots & 1\end{array}\right]\right\} n n a$ 


$$
\begin{aligned}
& C_{0}=\left[\begin{array}{cccc}
c_{1,1,0} & c_{1,2,0} & \cdots & c_{1, n u, 0} \\
\vdots & \vdots & \cdots & \vdots \\
c_{n y, 1,0} & c_{n y, 2,0} & \cdots & c_{n y, n u, 0}
\end{array}\right] \\
& C_{d}=\begin{array}{l}
\operatorname{diag}\left[c_{1,1,1} c_{1,1,2} \cdots c_{1,1, n a} c_{1,2,1} c_{1,2,2} \cdots c_{1,2, n a} \cdots c_{1, n u, 1} \cdots c_{1, n u, n a} \cdots c_{2,1,1} \cdots c_{2, n u, n a} \cdots\right. \\
\left.c_{n y, 1,1} \cdots c_{n y, 1,2} \cdots \cdots c_{n y, n u, n a}\right]
\end{array}
\end{aligned}
$$

sendo que $C_{0}$ e $C_{d}$ são obtidos através da expansão da resposta ao degrau no domínio de z em frações parciais.

\subsubsection{Controladores - QDMC e MMPC}

Tendo sido analisados os modelos de resposta ao degrau para a predição, faz-se necessário analisar os controladores, em específico os problemas de otimização que devem ser resolvidos, para que se tenha a seqüência de controle que deverá ser implementada no processo.

\subsubsection{Representação do erro nas predições}

Nesta abordagem de controlador, o modelo de predição das variáveis controladas para um sistema multivariável é representado pela (3.6) conforme apresentada em Odloak (1995).

$$
\bar{y}=y^{d}+S_{m n} \Delta u
$$

onde:

$\bar{y}$ - Vetor das predições das variáveis controladas após a aplicação de $\Delta u$ $y^{d}$ - Vetor das variáveis controladas antes da aplicação de $\Delta u$

$S_{m n}$ - Matriz dinâmica - $S_{m n}=\left[\begin{array}{cccc}S_{1} & 0 & \cdots & 0 \\ S_{2} & S_{1} & \cdots & 0 \\ \vdots & \vdots & \ddots & \\ S_{n c} & S_{n c-1} & \cdots & S_{1} \\ \vdots & \vdots & \cdots & \vdots \\ S_{n p} & S_{n p-1} & \cdots & S_{n p-n c+1}\end{array}\right] \quad S_{m n} \in \mathfrak{R}^{(n y . n p) x(n u . n c)}$ 
$\Delta u$ - Vetor das ações de controle - $\Delta u=[\Delta u(k) \Delta u(k+1) \cdots \Delta u(k+n c-1)]^{T}$ $\Delta u \in \mathfrak{R}^{(n u . n c) x(1)}$

$n c$ - horizonte de controle

Subtraindo $y^{S P}$ de ambos os membros do modelo de predição (3.6), definimos novas variáveis erro conforme apresentada a seguir.

$\bar{y}-y^{S P}=y^{d}-y^{S P}+S_{m n} \Delta u \Rightarrow e=e^{\prime}+S_{m n} \Delta u$

onde:

$y^{S P}$ - set-point para a variável controlada - $\quad y^{S P} \in \mathfrak{R}^{(n y . n p) x(1)}$

$e$ - erro entre os valores previstos e set-point - $\quad e \in \mathfrak{R}^{(n y . n p) x(1)}$

$e^{\prime}$ - erro sem nenhuma ação de controle futura - $\quad e^{\prime} \in \mathfrak{R}^{(n y . n p) x(1)}$

Sendo a expressão definida em (3.7) amplamente utilizado na definição do controlador QDMC conforme apresentado a seguir.

\subsubsection{QDMC com Targets para as entradas}

O controlador QDMC pode ser estendido para acomodar os targets para as variáveis manipuladas conforme apresentado por Zanin (2001) e Rotava e Zanin (2005). Este novo controlador é baseado na função objetivo definida em (3.8).

$$
\begin{aligned}
\min _{\Delta u} J= & \sum_{i=1}^{n p}\left[\left(y^{c}(k+i / k)-y^{s p}\right)^{T} Q\left(y^{c}(k+i / k)-y^{s p}\right)\right]+ \\
& \sum_{j=1}^{n c}\left[(\Delta u(k+j-1 / k))^{T} R(\Delta u(k+j-1 / k))\right]+ \\
& \sum_{j=1}^{n c}\left[\left(u(k+j-1 / k)-u_{s s}\right)^{T} R_{u}\left(u(k+j-1 / k)-u_{s s}\right)\right]
\end{aligned}
$$

onde:

$Q, R$ e $R_{u} \geq 0$

$y^{s p}$ - set-point para as variáveis controladas 
$u_{s s}$ - targets para as variáveis manipuladas

$Q$ - matriz diagonal de fatores de peso para as variáveis controladas $Q=\operatorname{diag}\left[q_{1} \ldots q_{n y}\right] Q \in \mathfrak{R}^{(n y) x(n y)}$

$R$ - matriz diagonal de fatores de supressão das ações de controle $R=\operatorname{diag}\left[r_{1} \ldots r_{n u}\right] R \in \mathfrak{R}^{(n u) x(n u)}$

$R_{u}$ - matriz diagonal de fatores para conduzir as variáveis manipuladas aos seus targets $-R_{u}=\operatorname{diag}\left[r u_{1} \ldots r u_{n u}\right] \quad R_{u} \in \mathfrak{R}^{(n u) \times(n u)}$

Expandindo a função objetivo (3.8) para todo o horizonte de predição $n p$ e horizonte de controle $n c$, tem-se:

$$
\begin{aligned}
\min _{\Delta u} J= & {\left[\left(\hat{y}^{c}(k+1 / k)-y^{s p}\right)^{T} Q\left(\hat{y}^{c}(k+1 / k)-y^{s p}\right)+\left(\hat{y}^{c}(k+1 / k)-y^{s p}\right)^{T} Q\left(\hat{y}^{c}(k+1 / k)-y^{s p}\right)\right.} \\
& \left.+\ldots+\left(\hat{y}^{c}(k+n p / k)-y^{s p}\right)^{T} Q\left(\hat{y}^{c}(k+n p / k)-y^{s p}\right)\right]+ \\
& {\left[(\Delta u(k / k))^{T} R(\Delta u(k / k))+(\Delta u(k+1 / k))^{T} R(\Delta u(k=1 / k))+\ldots+\right.} \\
& \left.(\Delta u(k+n c-1 / k))^{T} R(\Delta u(k+n c-1 / k))\right]+ \\
& {\left[\left(u(k / k)-u_{s s}\right)^{T} R_{u}\left(u(k / k)-u_{s s}\right)+\left(u(k+1 / k)-u_{s s}\right)^{T} R_{u}\left(u(k+1 / k)-u_{s s}\right)\right.} \\
& \left.+\ldots+\left(u(k+n c-1 / k)-u_{s s}\right)^{T} R_{u}\left(u(k+n c-1 / k)-u_{s s}\right)\right]
\end{aligned}
$$

Rearranjando na forma vetorial, tem-se:

$J(k)=e^{T} Q_{n p} e+\Delta u^{T} R_{n c} \Delta u+\left(u-u_{s s}\right)^{T} R_{u, n c}\left(u-u_{s s}\right)$

sendo:

$$
\begin{aligned}
& Q_{n p}=\operatorname{diag}[Q \ldots Q] \quad Q_{n p} \in \mathfrak{R}^{(n p . n y) x(n p . n y)} \\
& R_{n c}=\operatorname{diag}[R \ldots R] \quad R_{n c} \in \mathfrak{R}^{(n c . n u) x(n c . n u)} \\
& R_{u, n c}=\operatorname{diag}\left[R_{u} \ldots R_{u}\right] \quad R_{u, n c} \in \mathfrak{R}^{(n c . n u) x(n c . n u)}
\end{aligned}
$$


$e$ - vetor de erros após a aplicação do vetor de ações de controle $e=\left[\begin{array}{c}\hat{y}^{C}(k+1 / k)-y^{s p} \\ \vdots \\ \hat{y}^{C}(k+n p / k)-y^{s p}\end{array}\right] \quad e \in \mathfrak{R}^{(n p . n y) x(1)}$

$$
\begin{aligned}
& \Delta u=\left[\begin{array}{c}
\Delta u(k / k) \\
\vdots \\
\Delta u(k+n c-1 / k)
\end{array}\right] \quad \Delta u \in \mathfrak{R}^{(n c \cdot n u) x(1)} \\
& u=\left[\begin{array}{c}
u(k / k) \\
\vdots \\
u(k+n c-1 / k)
\end{array}\right] \quad u \in \mathfrak{R}^{(n c \cdot n u) x(1)}
\end{aligned}
$$

De forma alternativa, o vetor $u$ pode ser reescrito conforme apresentado em (3.10).

$$
u(k+l-1)=u(k-1)+\sum_{z=1}^{l} \Delta u(k+z-1)
$$

onde:

$l$ - varia de 1 a $\left(n_{c}-1\right)$

Assim, a equação (3.10) pode ser escrita para os instantes de $k$ até $k+n c-1$ conforme apresentado a seguir.

$$
\left[\begin{array}{c}
u(k / k) \\
u(k+1 / k) \\
\vdots \\
u(k+n c-1 / k)
\end{array}\right]=\left[\begin{array}{cccc}
I_{n c} & 0 & \cdots & 0 \\
I_{n c} & I_{n c} & \cdots & 0 \\
\vdots & \vdots & \ddots & \vdots \\
I_{n c} & I_{n c} & \cdots & I_{n c}
\end{array}\right]\left[\begin{array}{c}
\Delta u(k / k) \\
\Delta u(k+1 / k) \\
\vdots \\
\Delta u(k+n c-1 / k)
\end{array}\right]+\left[\begin{array}{c}
u(k-1 / k) \\
u(k-1 / k) \\
\vdots \\
u(k-1 / k)
\end{array}\right]
$$

Na forma compacta, a equação é dada por.

$u=N \Delta u+u^{\prime}$

onde:

$N \in \mathfrak{R}^{(n u . n c) x(n u . n c)}$ 
Assim, substituindo (3.7) e (3.11) em (3.9), tem-se:

$$
\begin{aligned}
& J(k)=\left(S_{m n} \Delta u+e^{\prime}\right)^{T} Q_{n p}\left(S_{m n} \Delta u+e^{\prime}\right)+\Delta u^{T} R_{n c} \Delta u \\
& +\left(N \Delta u+u^{\prime}-u_{s s}\right)^{T} R_{u, n c}\left(N \Delta u+u^{\prime}-u_{s s}\right)
\end{aligned}
$$

Considerando que $u_{d}=u^{\prime}-u_{s s}\left(u_{d} \in \mathfrak{R}^{(n c . n u) x 1}\right)$, tem-se:

$$
J(k)=\left(S_{m n} \Delta u+e^{\prime}\right)^{T} Q_{n p}\left(S_{m n} \Delta u+e^{\prime}\right)+\Delta u^{T} R_{n c} \Delta u+\left(N \Delta u+u_{d}\right)^{T} R_{u, n c}\left(N \Delta u+u_{d}\right)
$$

Para o desenvolvimento da operação matricial apresentada em (3.13) deve-se considerar as propriedades abaixo referentes à álgebra matricial.

$$
\begin{aligned}
& (A \cdot B)^{T}=B^{T} \cdot A^{T} \\
& (A+B)^{T}=A^{T}+B^{T}
\end{aligned}
$$

Assim, considerando, primeiramente, a expansão das matrizes e vetores presentes no primeiro termo relativo às variáveis controladas em (3.13),tem-se.

$$
\begin{aligned}
& \left(S_{m n} \Delta u+e^{\prime}\right)^{T} Q_{n p}\left(S_{m n} \Delta u+e^{\prime}\right)=\left(\Delta u^{T} S_{m n}^{T}+e^{\prime T}\right) Q_{n p}\left(S_{m n} \Delta u+e^{\prime}\right) \\
& =\left(\Delta u^{T} S_{m n}^{T} Q_{n p}+e^{\prime T} Q_{n p}\right)\left(S_{m n} \Delta u+e^{\prime}\right) \\
& =\Delta u^{T} S_{m n}^{T} Q_{n p} S_{m n} \Delta u+e^{\prime T} Q_{n p} S_{m n} \Delta u+\Delta u^{T} S_{m n}^{T} Q_{n p} e^{\prime}+e^{\prime T} Q_{n p} e^{\prime}
\end{aligned}
$$

Sendo $e^{\prime T} Q_{n p} S_{m n} \Delta u$ e $\Delta u^{T} S_{m n}^{T} Q_{n p} e^{\prime}$ expressões escalares, tem-se que $\Delta u^{T} S_{m n}^{T} Q_{n p} e^{\prime}=e^{\prime T} Q_{n p} S_{m n} \Delta u$. Assim, o primeiro termo de (3.13) pode ser escrito conforme apresentado em (3.14).

$$
\Delta u^{T} S_{m n}^{T} Q_{n p} S_{m n} \Delta u+2 e^{t^{T}} Q_{n p} S_{m n} \Delta u+e^{\prime T} Q_{n p} e^{\prime}
$$

Considerando o termo relativo aos targets para as variáveis controladas presentes em (3.13) , tem-se.

$$
\begin{aligned}
& \left(N \Delta u+u_{d}\right)^{T} R_{u, n c}\left(N \Delta u+u_{d}\right)=\left(\Delta u^{T} N^{T}+u_{d}^{T}\right) R_{u, n c}\left(N \Delta u+u_{d}\right)= \\
& \left(\Delta u^{T} N^{T} R_{u, n c}+u_{d}^{T} R_{u, n c}\right)\left(N \Delta u+u_{d}\right)= \\
& \Delta u^{T} N^{T} R_{u, n c} N \Delta u+\Delta u^{T} N^{T} R_{u, n c} u_{d}+u_{d}^{T} R_{u, n c} N \Delta u+u_{d}^{T} R_{u, n c} u_{d}
\end{aligned}
$$


Sendo $u_{d}^{T} R_{u, n c} N \Delta u$ e $\Delta u^{T} N^{T} R_{u, n c} u_{d}$ expressões escalares, tem-se que $\Delta u^{T} N^{T} R_{u, n c} u_{d}=u_{d}^{T} R_{u, n c} N \Delta u$. Assim, o terceiro termo de (3.13) pode ser escrito conforme apresentado em (3.15).

$$
\Delta u^{T} N^{T} R_{u, n c} N \Delta u+2 u_{d}^{T} R_{u, n c} N \Delta u+u_{d}^{T} R_{u, n c} u_{d}
$$

Substituindo (3.14) e (3.15) em (3.13), tem-se.

$$
\begin{aligned}
\min _{\Delta u} J & =\Delta u^{T}\left(S_{m n}{ }^{T} Q_{n p} S_{m n}+R_{n c}+N^{T} R_{u, n c} N\right) \Delta u \\
& +2\left(e^{T T} Q_{n p} S_{m n}+u_{d}{ }^{T} R_{u, n c} N\right) \Delta u+e^{\prime T} Q_{n p} e^{\prime}+u_{d}^{T} R_{u, n c} u_{d}
\end{aligned}
$$

Assim, reescrevendo (3.16) na forma compacta, tem-se (3.17).

$$
\min _{\Delta u} J=\Delta u^{T} H \Delta u+2 c_{f}^{T} \Delta u+c
$$

onde:

$$
\begin{aligned}
& H=S_{m n}{ }^{T} Q_{n p} S_{m n}+R_{n c}+N^{T} R_{u, n c} N \\
& c_{f}^{T}=e^{\prime T} Q_{n p} S_{m n}+u_{d}{ }^{T} R_{u, n c} N \\
& c=e^{\prime T} Q_{n p} e^{\prime}+u_{d}^{T} R_{u, n c} u_{d}
\end{aligned}
$$

Sendo o termo $c$ uma constante escalar, a mesma não altera a solução final caso seja eliminado da função objetivo. Da mesma forma, a solução desta função objetivo também não é alterada com a divisão por um fator de 2. Assim, (3.17) possui estrutura semelhante ao problema QP conforme apresentado em (3.18).

$$
\underset{\Delta u}{\operatorname{Min}} \Phi=\frac{1}{2} \Delta u^{T} H \Delta u+c_{f}^{T} \Delta u
$$

onde a matriz $H$ e o vetor $c_{f}^{T}$ são obtidas conforme apresentado em (3.17).

sujeito a: 


$$
\begin{aligned}
& {\left[\begin{array}{c}
I_{n c . n u} \\
-I_{n c . n u}
\end{array}\right] \Delta u \leq\left[\begin{array}{c}
N_{u} \Delta u_{\max } \\
N_{u} \Delta u_{\max }
\end{array}\right]} \\
& u_{\min } \leq u \leq u_{\max }
\end{aligned}
$$

onde:

$$
N_{u}=\left[\begin{array}{c}
I_{n u} \\
\vdots \\
I_{n u}
\end{array}\right] \quad N_{u} \in \mathfrak{R}^{(n c . n u) x 1}
$$

Desta forma, o problema de otimização definido para o QDMC pode ser resolvido por pacotes de QP encontrados comercialmente sem grandes dificuldades.

\subsubsection{MMPC para Sistemas com Vários Modelos}

Diferentemente da abordagem do controlador QDMC, o controlador MMPC resolve um problema de controle através da utilização de um conjunto de modelos os quais descrevem os diversos comportamentos do processo ao longo da região de operação do controlador.

O MMPC foi desenvolvido inicialmente por Almeida Neto (1999) utilizando a formulação em LMl's. Entretanto, devido a falta de pacotes computacionais que favoreçam a implementação desta abordagem inicial em um caso real, Porfírio (2001) desenvolveu uma solução alternativa a qual pode ser implementada com os pacotes de programação não-linear disponíveis no mercado.

Inicialmente, o problema de otimização parte da mesma função objetivo desenvolvida para o controlador QDMC. Assim, a função objetivo (3.17) é escrita novamente conforme apresentado a seguir.

$$
J(k)=\Delta u^{T} H \Delta u+2 c_{f}^{T} \Delta u+c
$$

onde:

$$
\begin{aligned}
& H=S_{m n}{ }^{T} Q_{n p} S_{m n}+R_{n c} \\
& c_{f}^{T}=e^{\prime T} Q_{n p} S_{m n}
\end{aligned}
$$


$c=e^{\prime T} Q_{n p} e^{\prime}$

Entretanto, uma ressalva deve ser feita, pois a função objetivo em (3.19) não apresenta o termo referente aos targets para as variáveis manipuladas. Isto é feito para que a abordagem analisada aqui para o controlador MMPC seja a mesma do controlador de Porfírio (2001) o qual não havia analisado o comportamento do controlador em uma estrutura de duas camadas.

A partir desta função objetivo, Porfírio et al. (2003) formulam o problema de otimização para o controlador MMPC conforme apresentado na (3.20).

$\min _{\Delta u, \gamma} \gamma$

sujeito a:

$$
\begin{aligned}
& \Delta u^{T} H_{i} \Delta u+2 c_{f, i}{ }^{T} \Delta u+c_{i}-\gamma<0 \\
& i=1, \ldots, L \\
& -\Delta u_{\max } \leq \Delta u(k+j / k) \leq \Delta u_{\max } \\
& u_{\min } \leq u(k+j / k) \leq u_{\max } \\
& j=1, \ldots, n_{c}
\end{aligned}
$$

Conforme pode ser visto acima, o problema de otimização para o controlador MMPC apresenta uma função objetivo linear e restrições lineares e não lineares que são quadráticas em $\Delta u$, portanto um problema não-linear, que não pode ser resolvido com os pacotes computacionais de programação linear (LP) ou quadrática (QP).

É possível verificar, também, que o problema de otimização leva em conta a função objetivo definida em (3.19) de tal forma que cada um dos $L$ modelos do processo participe como restrição.

Conforme apresentado neste problema de otimização, as ações de controle são calculadas minimizando a função objetivo para o pior modelo do processo utilizado. Verifica-se também que o parâmetro $\gamma$ participa da solução do problema como uma incógnita juntamente com a seqüência de ações de controle que devem ser calculadas. 


\subsubsection{Estratégia de Controle por Faixa}

Assim, tendo sido definido o problema de otimização para o cálculo das ações de controle, deve-se definir também a estratégia a ser utilizada para verificar se o processo está sob controle e senão tomar alguma ação. Neste caso, entende-se como ação a necessidade de otimizar uma nova seqüência de ações de controle para que as variáveis controladas estejam em uma faixa segura de operação.

Esta faixa, naturalmente, é definida pelos limites das variáveis controladas dados por $y_{\min }$ e $y_{\max }$, sendo limite mínimo e limite máximo, respectivamente.

A estratégia de controle por faixa, apresentada a seguir, é formulada conforme apresentado em Odloak (2005).

Devem-se analisar os valores do vetor $\hat{y}^{C}(k+n / k)$ em cada um dos $n p$ instantes de predição verificando se estão entre os limites, acima do limite máximo ou abaixo do limite mínimo. A seguir são apresentadas estas análises com as respectivas ações a serem tomadas.

$>$ Se $y_{\min } \leq \hat{y}^{C}(k+n / k) \leq y_{\max }$, a saída $\hat{y}^{C}(k+n / k)$ deve ser ignorada (removida dos cálculos de controle) no instante de predição $k+n$. Para tanto, o parâmetro de sintonia $q_{i}$ que define a matriz $Q$ deve ser zero e $e^{\prime}(k+n / k)=-\hat{y}^{C}(k+n / k)$.

$>$ Se $\hat{y}^{C}(k+n / k)>y_{\max }$, a variável controlada $\hat{y}^{C}(k+n / k)$ deve ser trazida para o seu limite superior e assim $e^{\prime}(k+n / k)=\hat{y}^{c}(k+n / k)-y_{\max }$ no instante de predição $k+n$. Neste caso, $q_{i}$ será dado por parâmetro de sintonia definido previamente.

$>$ Se $\hat{y}^{C}(k+n / k)<y_{\min }$, a variável controlada $\hat{y}^{C}(k+n / k)$ deve ser trazida para o seu limite inferior e assim $e^{\prime}(k+n / k)=\hat{y}^{c}(k+n / k)-y_{\min }$ no instante de predição $k+n$. Neste caso, $q_{i}$, também, será dado por parâmetro de sintonia definido previamente. 


\section{METODOLOGIA PROPOSTA PARA O MPC ADAPTATIVO}

Neste capítulo será apresentada a metodologia do controlador adaptativo, tema da presente dissertação. Na seção 4.1 é apresentado o exemplo de sistema a ser considerado nas simulações do presente trabalho. Na seção 4.2 é desenvolvido o procedimento de identificação do modelo de predição para a inclusão no controlador MPC adaptativo. Na seção 4.3 é apresentado o tipo de sinal o qual será utilizado para a excitação do processo. Finalmente, na seção 4.4 será realizada a caracterização da excitação persistente para avaliar se a perturbação injetada foi suficiente para identificar o novo modelo.

\subsection{Exemplo de Sistema - Coluna Depropanizadora}

Nesta seção está sendo apresentado o processo que será utilizado para a aplicação da metodologia proposta de controle adaptativo. Este processo foi estudado por Porfírio (2001) e o P\&I (piping and instrumentation) é apresentado a seguir. Neste fluxograma, está sendo representado, também, o controlador MPC conforme será descrito posteriormente.

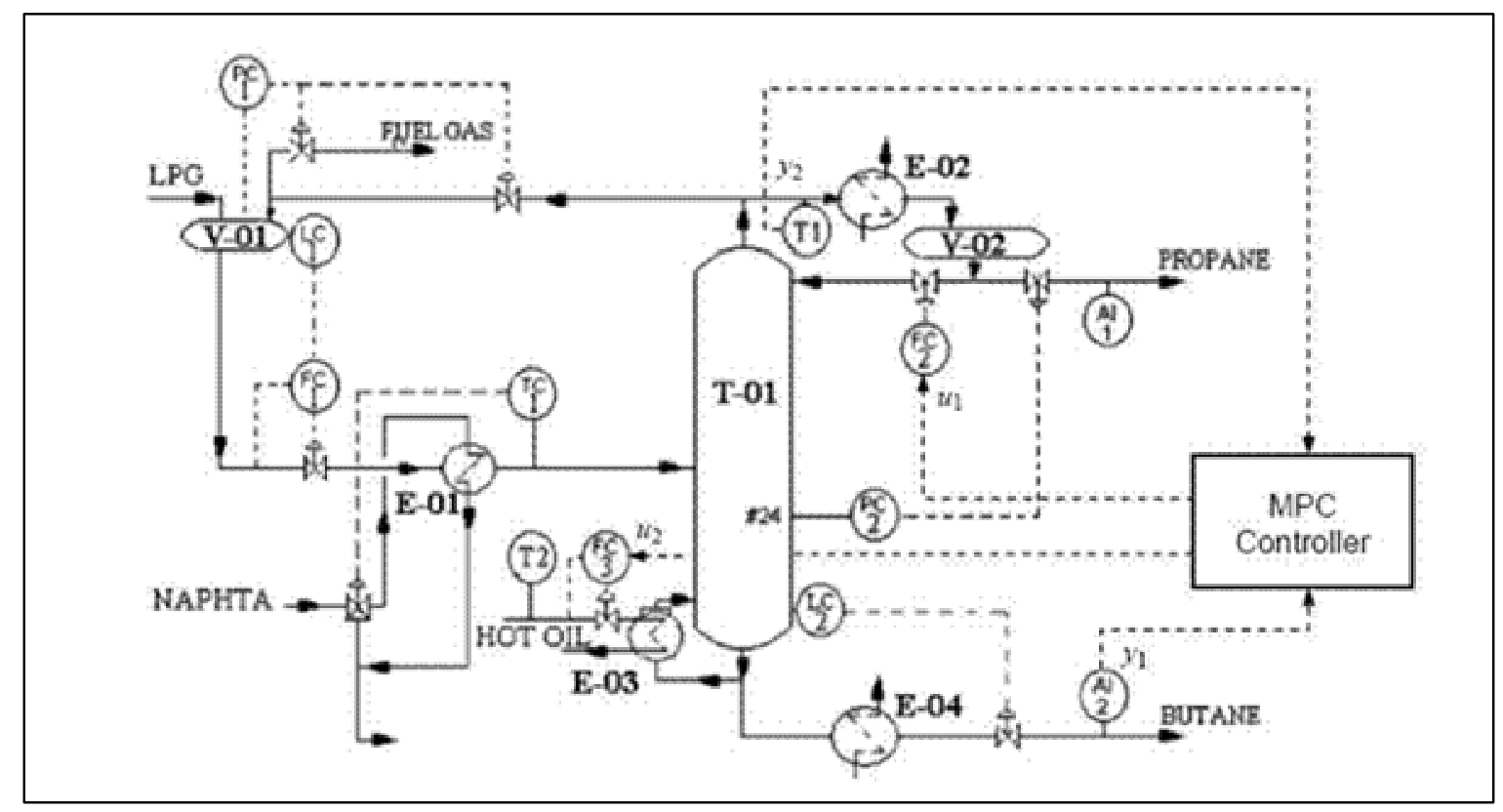

Figura 4-1 - P\&I para a coluna depropanizadora (Ballin, 2008) 
Neste processo, a coluna depropanizadora é responsável pela separação dos componentes leves (mais voláteis) tais como $\mathrm{C}_{3}$ dos componentes pesados (menos voláteis) tais como $\mathrm{C}_{4}$. A corrente de carga é gás liquefeito de petróleo (GLP) proveniente de uma coluna debutanizadora.

No P\&l apresentado, T-01 é a coluna de destilação, E identifica os trocadores de calor e $\mathrm{V}$ indica vasos de processo. A corrente de topo é composta basicamente por compostos $\mathrm{C}_{3}$ e a corrente de fundo, por $\mathrm{C}_{4}$. Al1 e Al2 são analisadores que inferem o teor de $\mathrm{C}_{4}$ no topo e $\mathrm{C}_{3}$ no fundo, respectivamente.

Conforme apresentado, também, no P\&l, o controlador MPC apresenta como variáveis controladas $y_{1}$ e $y_{2}$ as quais são, respectivamente, a composição molar de $\mathrm{C}_{3}$ na corrente de fundo (\%) e a temperatura no primeiro estágio da primeira seção de topo da coluna $\left({ }^{\circ} \mathrm{C}\right)$. Enquanto que as variáveis manipuladas $u_{1}$ e $u_{2}$ são as vazões de refluxo no topo da coluna $\left(\mathrm{m}^{3} / \mathrm{d}\right)$ e de fluido de aquecimento para o refervedor no fundo da coluna $\left(\mathrm{m}^{3} / \mathrm{d}\right)$.

Em relação às especificações de operação, tem-se que para a corrente de topo, o valor de compostos pesados (C4+) deve ser no máximo de $3 \%$. Enquanto que para a corrente de fundo, o valor de compostos leves (C3-) deve ser de no máximo de $3 \%$.

\subsubsection{Modelos do Processo e Matriz de Resposta ao Degrau}

Para a presente proposta serão utilizados os modelos de processo identificados por Porfírio (2001) conforme apresentados no Anexo A. Estes modelos são apresentados através dos coeficientes da função transferência no domínio de Laplace conforme representada a seguir.

$$
G(s)=\frac{b_{0}+b_{1} s}{s^{2}+a_{1} s+a_{2}} e^{-s \theta}
$$

Para a obtenção dos modelos, Porfírio (2001) considerou os pontos de operação da coluna conforme pode ser verificada pela Figura 4-2. 


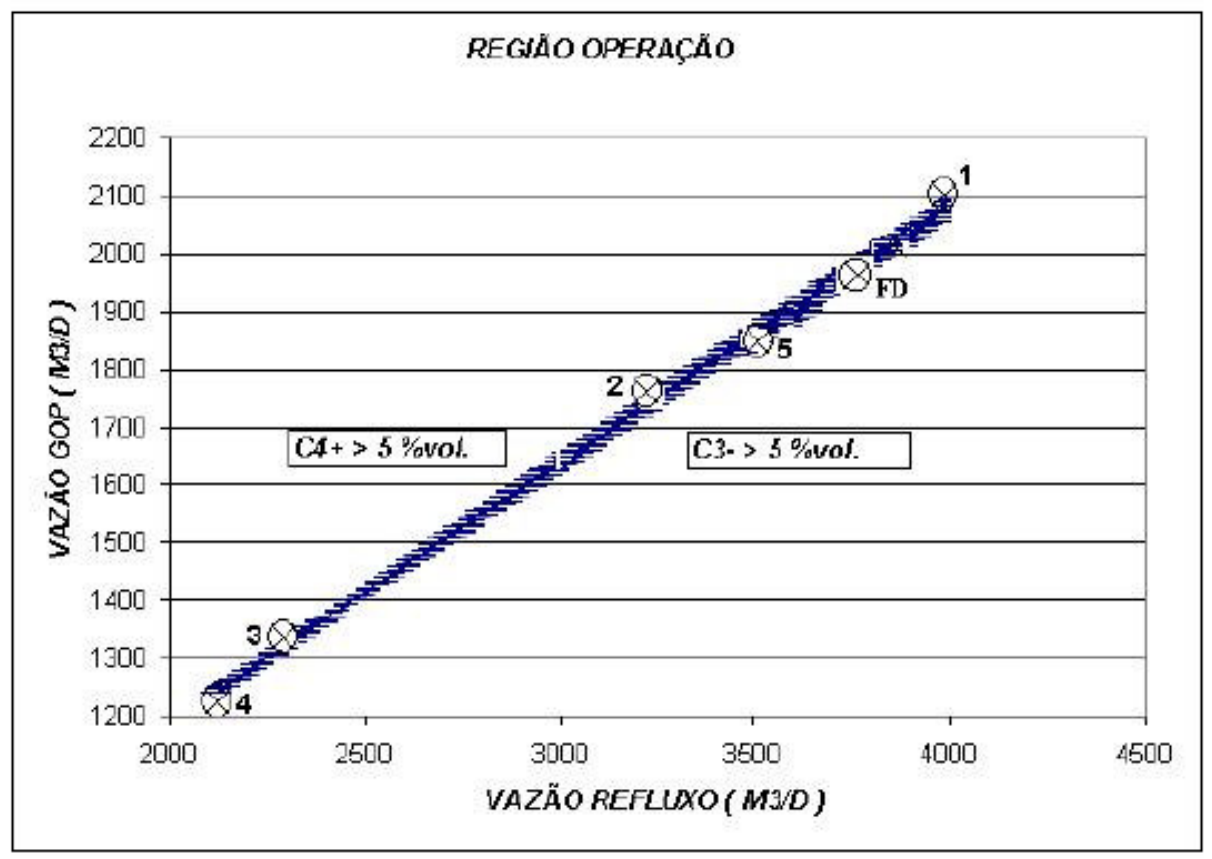

Figura 4-2 - Pontos de operação da depropanizadora (Porfírio, 2001)

De uma forma geral, cada um dos modelos obtidos são vértices de um politopo de tal forma que qualquer outro modelo correspondente a qualquer outra condição seria obtido pela combinação convexa deste conjunto de modelos (Porfírio, 2001).

\subsection{Controlador Adaptativo}

Conforme apresentado na seção 1.2, referente ao objetivo do presente trabalho, é considerada a proposta de um controlador adaptativo. Para tanto, deve ser considerado o modelo definido na seção 3.2.1 e que está sendo reproduzido a seguir.

$$
\begin{aligned}
& x(k+1)=M \cdot x(k)+S \cdot \Delta u(k) \\
& y(k)=C \cdot x(k)
\end{aligned}
$$

Para sistemas representados na forma definida em (4.2), toda a incerteza concentrase na matriz $S$.Desta forma, assumindo como hipótese que o processo sob controle possui $L$ modelos identificados representados pelas matrizes $S_{1}, S_{2}, \cdots, S_{L}$ e que cada um destes modelos define um politopo, pode-se escrever: 
$S=\sum_{z=1}^{L} \lambda_{z} S_{z}$

sujeita a:

$\sum_{z=1}^{L} \lambda_{z}=1, \lambda_{z} \geq 0, z=1, \ldots, L$

Assim, o efeito das ações de controle na predição da saída pode ser obtido, considerando a combinação dos $L$ modelos do processo, conforme apresentado em (4.4).

$\tilde{y}(k+n / k)=y(k+n / k)+\left(\sum_{z}^{L} \lambda_{z} S_{z}\right) \Delta u(k)$

onde:

$\tilde{y}(k+n / k)$ - vetor das variáveis controladas

Considerando somente a predição em $k+1$, tem-se.

$\tilde{y}(k+1 / k)=y(k+1 / k)+\left(\sum_{z=1}^{L} \lambda_{z} S_{z, 1}\right) \Delta u(k)$

onde:

$S_{z, 1}$ - primeiro coeficiente da resposta ao degrau para o modelo $z$

Assim, o problema de otimização para determinar o conjunto de coeficientes $\lambda_{i}$ os quais definem o modelo usado no controlador adaptativo será dado em (4.6).

$\min _{\lambda} \sum_{v=1}^{T_{\text {ident }}}[\tilde{y}(k-v+1 / k-v)-y(k-v+1)]^{2}$

onde:

$y(k-v+1)$ - saída lida da planta no instante $k-v+1$ 
Substituindo (4.5) em (4.6), vemos que $\lambda_{i}$ se torna explícito no problema de otimização.

$$
\min _{\lambda} \sum_{v=1}^{T_{\text {idenn }}}\left[y(k-v+1 / k-v)+\sum_{z=1}^{L} \lambda_{z} S_{z, 1} \Delta u(k-v)-y(k-v+1)\right]^{2}
$$

sujeito a:

$\sum_{z=1}^{L} \lambda_{z}=1$ e $\lambda_{z} \geq 0$

Para resolver este problema de otimização será empregado o pacote computacional

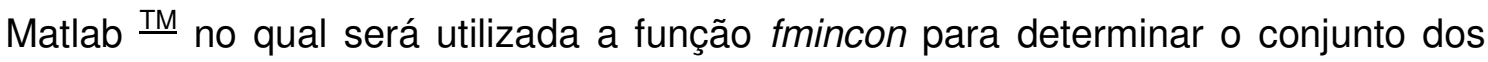
coeficientes $\lambda_{i}$ através da solução do problema de otimização definido em (4.7).

Vale ressaltar que se trata de um problema de otimização convexo, ou seja, com uma única solução.

\subsubsection{Ações de Controle}

Para a determinação da seqüência de ações de controle o controlador adaptativo usa o modelo obtido em (4.3) associado à abordagem definida na seção 3.2.3.2 para o QDMC com Targets para as entradas considerando uma estrutura de controle em duas camadas. Em função disto, o controle adaptativo será denominado AQDMC.

\subsection{Excitação para a Identificação de Modelos para o Controlador Adaptativo}

Foi considerada a identificação em malha fechada através do método direto para a geração dos dados com a aplicação de uma excitação externa através de um sinal que será do tipo de seqüência binária pseudo-randômica a ser implementada através do algoritmo GBN.

\subsubsection{GBN}

O algoritmo GBN permite gerar um sinal estocástico o qual é alterado randomicamente entre dois níveis fixos em pontos discretos no tempo (Tulleken 
1990, apud Zhu 2001). Para a sua implementação está sendo considerada a equação abaixo.

$$
\text { prob }=\frac{T}{E T_{S W}^{*}}
$$

onde:

prob - probabilidade para alterar entre os dois níveis fixos

$T$ - período de amostragem

$E T_{S W}^{*}$ - tempo médio decorrido entre duas alterações consecutivas.

sendo $E T_{S W}^{*}=0.98 * \frac{T_{s s}}{3}$

onde:

$T_{s s}$ - tempo de estabilização

Pelo procedimento proposto por Zhu (2001) e conhecimento prévio do processo, são gerados dois sinais GBN independentes de magnitude \pm 1 com tempo médio de alteração definidos em $E T_{S W}^{*}$.

Abaixo são apresentadas as principais vantagens em se utilizar o sinal GBN durante a excitação do sistema:

> Permite definir os níveis de excitação através dos níveis mínimo (-1) e máximo $(+1)$;

Manipular a distribuição através da probabilidade de alteração entre os níveis;

Manipular o tempo médio decorrido entre duas alterações consecutivas através de $T_{s S}$;

\subsubsection{Camada de Otimização}

A perturbação gerada pelo sinal GBN será introduzida na camada estática de tal forma que a perturbação será propagada para a camada dinâmica através do target 
$u_{s s}$ gerado, perturbando diretamente as variáveis manipuladas do processo. Assim, a função objetivo do problema de otimização na camada estática conforme formulada na seção 3.1 passa a ser escrito conforme apresentado a seguir (Sotomayor et al., 2008).

$$
\min _{\Delta u_{s s}, \delta_{y}}\left(\mathrm{~W}_{\mathrm{exc}} \mathrm{W}_{1}\right)^{T} \Delta u_{s s}+\left|\mathrm{W}_{2}^{\mathrm{T}} \delta_{y}\right|
$$

onde:

$\mathrm{W}_{\text {exc }}$ - vetor de seqüência binária $(-1$ e +1$)$ pseudo-randômica definida pelo sinal GBN

Como pode ser analisado, o problema de otimização será resolvido após a aplicação do valor -1 ou +1 a depender do valor no vetor $\mathrm{W}_{\mathrm{exc}}$.

Neste mesmo problema de otimização, o ganho $K$ será o mesmo do modelo definido pelo controlador AQDMC o qual é determinado pela ponderação de $\lambda_{i} \mathrm{em}$ cada um dos $L$ ganhos dos modelos. Desta forma, o novo ganho é representado pela (4.11).

$$
\begin{aligned}
& K=\sum_{i=1}^{L} \lambda_{i} K_{i} \\
& \lambda_{i} \geq 0, i=1, \ldots, L
\end{aligned}
$$

onde:

$L$ - número de modelos

$K_{i}$ - ganho do processo para o modelo z

$\lambda_{i}$ - coeficiente $i$ referente ao modelo $z$

\subsection{Caracterização da Excitação Persistente}

Em paralelo ao processo de identificação através da aplicação do sinal GBN, é proposto também a caracterização da excitação persistente. Através desta caracterização é possível dizer que o processo foi suficientemente excitado, 
permitindo assim obter a melhor combinação dos $\lambda_{i}$ conforme definido pelo problema de otimização (4.6).

Para tanto, considerando a função de transferência apresentada em (4.1) convertida para o domínio Z, tem-se:

$G(z)=\frac{y(z)}{u(z)}=\frac{b_{1} z^{-1-\frac{\theta}{T}}+b_{2} z^{-2-\frac{\theta}{T}}}{1+a_{1} z^{-1}+a_{2} z^{-2}}$

Fazendo a inversão para o domínio no tempo, para o instante k, tem-se:

$$
y(k)+a_{1} y(k-1)+a_{2} y(k-2)=b_{1} u\left(k-1-\frac{\theta}{T}\right)+b_{2} u\left(k-2-\frac{\theta}{T}\right)
$$

Rearranjando e fazendo com que $y(k)=\hat{y}(k)$ e desenvolvendo para cada um dos $\mathrm{i}$ modelos que compõe o grupo de modelos identificados, tem-se:

$$
\hat{y}_{i}(k)=-a_{1}{ }^{i} y(k-1)-a_{2}{ }^{i} y(k-2)+b_{1}{ }^{i} u\left(k-1-\frac{\theta^{i}}{T}\right)+b_{2}{ }^{i} u\left(k-2-\frac{\theta^{i}}{T}\right)
$$

onde:

$a_{1}^{i}, a_{2}^{i}, b_{1}^{i}$ e $b_{2}^{i}$ - coef. da função transferência no domínio de $\mathrm{k}$ para cada modelo i $\hat{y}_{i}(k)$ - predição das variáveis controladas no instante $k$ para o modelo $\mathrm{i}$ $y(k-1)$ - leituras das variáveis controladas no instante $k-1$ $y(k-2)$ - leituras das variáveis controladas no instante $k-2$ $\theta^{i}$ - tempo morto para o modelo i $T$ - período de amostragem $u\left(k-1-\frac{\theta_{i}}{T}\right)$ - leitura da variável manipulada no instante $k-1-\frac{\theta_{i}}{T}$ $u\left(k-2-\frac{\theta_{i}}{T}\right)$ - leitura da variável manipulada no instante $k-2-\frac{\theta_{i}}{T}$

Por se tratar de uma combinação entre os $L$ modelos, deve ser realizada a combinação entre cada um deles através do coeficiente $\lambda_{i}$ o qual representa o peso 
do modelo i na composição da predição da variável controlada $\hat{y}(k)$, conforme apresentado em (4.13)

$$
\begin{aligned}
\hat{y}(k)= & {\left[-\lambda_{1} a_{1}{ }^{1} y(k-1)-\lambda_{1} a_{2}{ }^{1} y(k-2)+\lambda_{1} b_{1}{ }^{1} u\left(k-1-\frac{\theta^{1}}{T}\right)+\lambda_{1} b_{2}{ }^{1} u\left(k-2-\frac{\theta^{1}}{T}\right)\right]+} \\
& {\left[-\lambda_{2} a_{1}{ }^{2} y(k-1)-\lambda_{2} a_{2}{ }^{2} y(k-2)+\lambda_{2} b_{1}{ }^{2} u\left(k-1-\frac{\theta^{2}}{T}\right)+\lambda_{2} b_{2}{ }^{2} u\left(k-2-\frac{\theta^{2}}{T}\right)\right]+} \\
& \cdots \\
& {\left[-\lambda_{L} a_{1}{ }^{L} y(k-1)-\lambda_{L} a_{2}{ }^{L} y(k-2)+\lambda_{L} b_{1}{ }^{L} u\left(k-1-\frac{\theta^{L}}{T}\right)+\lambda_{L} b_{2}{ }^{L} u\left(k-2-\frac{\theta^{L}}{T}\right)\right] }
\end{aligned}
$$

Subtraindo $y(k)$ de ambos os membros da expressão (4.13) e definindo o erro $\bar{e}(k)=\hat{y}(k)-y(k)$, tem-se:

$$
\begin{aligned}
\bar{e}(k)= & {\left[-\lambda_{1} a_{1}{ }^{1} y(k-1)-\lambda_{1} a_{2}{ }^{1} y(k-2)+\lambda_{1} b_{1}{ }^{1} u\left(k-1-\frac{\theta^{1}}{T}\right)+\lambda_{1} b_{2}{ }^{1} u\left(k-2-\frac{\theta^{1}}{T}\right)\right]+} \\
& {\left[-\lambda_{2} a_{1}{ }^{2} y(k-1)-\lambda_{2} a_{2}{ }^{2} y(k-2)+\lambda_{2} b_{1}{ }^{2} u\left(k-1-\frac{\theta^{2}}{T}\right)+\lambda_{2} b_{2}{ }^{2} u\left(k-2-\frac{\theta^{2}}{T}\right)\right]+} \\
& \cdots \\
& {\left[-\lambda_{L} a_{1}{ }^{L} y(k-1)-\lambda_{L} a_{2}{ }^{L} y(k-2)+\lambda_{L} b_{1}{ }^{L} u\left(k-1-\frac{\theta^{L}}{T}\right)+\lambda_{L} b_{2}{ }^{L} u\left(k-2-\frac{\theta^{L}}{T}\right)\right]-y(k) }
\end{aligned}
$$

Rearranjando a equação anterior obtém-se:

$$
\bar{e}(k)=\sum_{z=1}^{L} \lambda_{z}\left[\begin{array}{l}
-a_{1}^{z} \cdot y(k-1)-a_{2}^{z} \cdot y(k-2)+b_{1}^{z} \cdot u\left(k-1-\frac{\theta^{z}}{T}\right) \\
+b_{2}^{z} \cdot u\left(k-2-\frac{\theta^{z}}{T}\right)
\end{array}\right]-y(k)
$$

Substituindo a expressão $-a_{1}^{z} y(k-1)-a_{2}^{z} y(k-2)+b_{1}^{z} u\left(k-1-\frac{\theta^{z}}{T}\right)+b_{2}^{z} u\left(k-2-\frac{\theta^{z}}{T}\right)$ por $c_{z}(k)$, tem-se:

$$
\begin{aligned}
& \bar{e}(k)=\sum_{z=1}^{L} \lambda_{z} c_{z}(k)-y(k) \Rightarrow \\
& \bar{e}(k)=\lambda_{1} c_{1}(k)+\lambda_{2} c_{2}(k)+\cdots+\lambda_{L} c_{L}(k)-y(k) \Rightarrow \\
& \bar{e}(k)=\lambda^{T} c(k)-y(k)
\end{aligned}
$$


onde:

$c(k)$ - vetor de coeficientes - $c(k)=\left[c_{1}(k) c_{2}(k) \ldots c_{L}(k)\right]^{T}$

$\lambda^{T}$ - vetor de coeficientes para a combinação - $\lambda=\left[\begin{array}{lll}\lambda_{1} & \lambda_{2} & \cdots\end{array} \lambda_{L}\right]^{T}$

Aplicando (4.15) desde o instante $k-1$ até $k-T_{\text {ident }}$, tem-se:

$\bar{e}(k-1)=\lambda^{T} c(k-1)-y(k-1)$

$\vdots$

$\bar{e}\left(k-T_{\text {ident }}\right)=\lambda^{T} c\left(k-T_{\text {ident }}\right)-y\left(k-T_{\text {ident }}\right)$

Passando para a forma matricial obtém-se a expressão abaixo.

$\bar{e}=\lambda^{T} \cdot \bar{A}-\bar{y}$

onde:

$\bar{e}$ - vetor de erros desde $k-1$ até $k-T_{\text {ident }}$

$\bar{y}$ - vetor de leitura da variável controlada na planta - $\bar{y}=\left[y(k-1)^{T} \cdots y\left(k-T_{\text {ident }}\right)^{T}\right]^{T}$

$\bar{A}$ - matriz de coeficientes - $\bar{A}=\left[c(k-1)^{T} \ldots c\left(k-T_{\text {ident }}\right)^{T}\right]^{T}$

Multiplicando ambos os membros da equação (4.16) por $\bar{A}^{T}$ e isolando o vetor dos parâmetros $\lambda$, tem-se:

$\lambda=\left(\bar{A}^{T} \cdot \bar{A}\right)^{-1}\left[\bar{A}^{T} \cdot \bar{e}+\bar{A}^{T} \cdot \bar{y}\right]$

que seria a solução ótima do problema (4.7) se não houvesse restrições.

Assim, pode-se garantir que o sistema foi suficientemente excitado observando os autovalores da matriz $\left(\bar{A}^{T} \cdot \bar{A}\right)$. Se os autovalores forem todos maiores que um certo $\xi>0$, então o vetor $\lambda$ pode ser identificado através da solução do problema definido em (4.7). 


\section{VALIDAÇÃO DA METODOLOGIA}

Neste capítulo serão apresentadas as metodologias para a validação dos resultados obtidos com a estratégia proposta. Na seção 5.1 está sendo proposta a simulação do MMPC como forma de validar o controlador adaptativo proposto. Na seção 5.2 está sendo proposta a utilização da metodologia de identificação de sistemas para identificar o modelo da planta na forma de um modelo ARX e posteriormente utilizálo para comparar com o modelo obtido pela metodologia proposta do controlador adaptativo.

\subsection{Comparação com o MMPC}

Estão sendo propostos testes para comparar o desempenho, frente a alteração nas faixas de controle, entre o controlador AQDMC proposto e o MMPC de Porfírio et al. (2003).

Para tanto, será utilizado o problema de otimização definida na seção 3.2.3.3 onde é apresentado o MMPC para Sistemas com Vários Modelos.

Finalmente, para resolver este problema de otimização será empregado o pacote

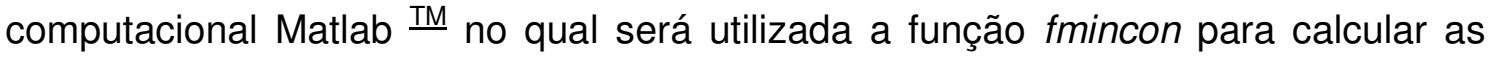
ações de controle para o problema de otimização não-linear definido para o controlador MMPC.

\subsection{Comparação de modelos}

Está sendo proposta, também, para a comparação dos resultados obtidos com a nova metodologia de identificação usada no controlador adaptativo AQDMC com os resultados obtidos quando se identifica diretamente o modelo da planta através de um modelo ARX.

Segundo Ljung (1999), a identificação de sistemas trata do problema da construção de modelos matemáticos de sistemas dinâmicos a partir de dados observados. Assim, será utilizada a identificação de sistemas para levantar o modelo que mais se aproxima da planta. A este modelo será dado o nome de modelo identificado. 
Antes de realizar a identificação propriamente dita deve-se preparar os dados utilizando a média e desvio padrão do próprio conjunto de valores. A seguir são apresentadas as fórmulas para a normalização destes dados.

$$
\begin{aligned}
& u_{n}=\frac{(u(k)-\bar{u})}{\sigma_{u}} \\
& y_{n}=\frac{(y(k)-\bar{y})}{\sigma_{y}}
\end{aligned}
$$

onde:

$u_{n}$ e $y_{n}$-variáveis manipuladas e controladas após rotina de normalização.

$\bar{u}$ e $\bar{y}$ - valores médios

$\sigma_{u}$ e $\sigma_{y}$ - desvio padrão

Com a base de dados preparada é possível realizar a identificação. Para tanto será utilizada a estrutura ARX conforme representada a seguir:

$$
A(q) y(k)=B(q) u(k)+e(k)
$$

Onde:

$u(k)$ e $y(k)$ - variáveis manipuladas e controladas instantânea.

$e(k)$ - ruído branco

$A(q) B(q)$ - Polinômios de ajuste do modelo

O segundo modelo a ser considerado nesta validação consiste no modelo obtido pela metodologia proposta do controlador adaptativo conforme apresentado na seção 4. A este modelo será dado o nome de modelo combinado.

Após a obtenção dos modelos - identificado e combinado, serão realizados os testes conforme apresentado a seguir: 
Degrau - será utilizada a função step do Matlab para comparar a resposta ao degrau tanto no modelo identificado quanto no combinado e, também, na planta.

> Simulação - através da função de simulação ( $\operatorname{sim}$ ) presente no Matlab será possível comparar as variáveis controladas obtidas pelos dois modelos identificado e combinado, a partir das entradas armazenadas durante excitação da planta.

Finalmente, esta fase de testes será encerrada com a aplicação de dois critérios os quais são - FIT e $R^{2}$, para a validação tanto do modelo identificado quanto do modelo combinado em relação aos dados da planta. O critério FIT apresenta o percentual de ajuste dos dados de saída do modelo às saídas medidas, enquanto que o coeficiente $R^{2}{ }_{T}$ indica o quanto da saída do modelo pode explicar o comportamento da saída do sistema (Sotomayor et al, 2008). Estes dois critérios são apresentados a seguir.

$F I T=\left(1-\frac{|y(t)-\hat{y}(t)|}{|y(t)-\bar{y}|}\right) x 100$

$R_{T}^{2}=1-\frac{\operatorname{var}[y(t)-\hat{y}(t)]}{\operatorname{var}[y(t)]}$

onde:

$y(t)$ - saídas observadas/medidas

$\hat{y}(t)$ - saídas estimadas do modelo

$\bar{y}(t)$ - valor médio das saídas medida/observadas.

var - variância de $y(t)-y(t)$ e $y(t)$.

Enquanto que para os cálculos propriamente dito dos critérios FIT e $R^{2}$ T será utilizado o toolbox CONTSID (Garnier et al., 2006) por apresentar métodos com melhor precisão para a realização destes cálculos. 


\section{RESULTADOS E DISCUSSÃO}

Neste capítulo são apresentados os resultados da metodologia proposto para o controlador MPC adaptativo. Na seção 6.1, a simulação de um controlador QDMC é realizada utilizando o modelo FD. Na seção 6.2, os modelos de Porfírio (2001) são analisados através do ganho, tempo de estabilização e sinal de excitação para a camada estática. Na seção 6.3, os parâmetros do controlador adaptativo relativos ao horizonte de otimização e tempo de experimento são analisados. Na seção 6.4, a metodologia de controlador adaptativo é aplicada para o caso real da coluna depropanizadora. Finalmente, na seção 6.5, a metodologia de controle adaptativo é validada através da comparação da performance com o controlador MMPC e identificação direta do modelo da planta através de um ARX.

\subsection{Caso Real - Coluna Depropanizadora}

Inicialmente, é apresentado o resultado da simulação do controlador QDMC utilizando o modelo FD (Tabela A-6) para o controlador e o modelo 1 (Tabela A-1) para a planta, ambos definidos no Anexo $A$.

Para a simulação está sendo considerada a sintonia do controlador conforme apresentado na Tabela 6-1. Para a camada de otimização, será considerada a sintonia conforme apresentado na Tabela 6-2.

Tabela 6-1 - Sintonia do Controlador - Camada dinâmica

\begin{tabular}{|c|c|c|c|}
\hline Parâmetro & Valor & Parâmetro & Valor \\
\hline ny & 2 & $\mathrm{R}_{\mathrm{u}}$ & $\operatorname{diag}\left[\begin{array}{ll}10 & 10\end{array}\right]$ \\
\hline $\mathrm{nu}$ & 2 & $\mathrm{u}_{\max }$ & {$\left[\begin{array}{lll}5450 & 4000\end{array}\right]^{\top}$} \\
\hline $\mathrm{nh}$ & 120 & $\mathrm{u}_{\min }$ & {$\left[\begin{array}{ll}1000 & 1000\end{array}\right]^{\top}$} \\
\hline$n_{p}$ & 60 & $\Delta u_{\max }$ & {$\left[\begin{array}{ll}10 & 10\end{array}\right]^{\top}$} \\
\hline $\mathrm{n}_{\mathrm{c}}$ & 2 & $y_{\max }$ & {$\left[\begin{array}{ll}0.7 & 48\end{array}\right]^{\top}$} \\
\hline $\mathrm{T}$ & 1 & $y_{\min }$ & {$\left[\begin{array}{ll}0.7 & 48\end{array}\right]^{\top}$} \\
\hline $\mathrm{R}$ & diag $\left[\begin{array}{lll}1.5 & 1.5\end{array}\right] \times 0.5$ & u_0 & {$\left[\begin{array}{ll}3160 & 1731\end{array}\right]^{\top}$} \\
\hline$Q$ & $\operatorname{diag}\left[\begin{array}{ll}1.3 & 1.2\end{array}\right]$ & y_0 & {$\left[\begin{array}{ll}0.7 & 48\end{array}\right]^{\top}$} \\
\hline
\end{tabular}


Tabela 6-2 - Sintonia do Otimizador - Camada Estática

\begin{tabular}{|l|l|l|l|}
\hline Parâmetro & Valor & Parâmetro & Valor \\
\hline$W_{1}$ & {$[1-1]^{\top}$} & $W_{2}$ & $\operatorname{diag[2~2]~}$ \\
\hline
\end{tabular}

Basicamente, será aplicada a perturbação no set-point de cada uma das variáveis controladas conforme apresentada na Tabela 6-3.

Tabela 6-3 - Desvio aplicados na simulação

\begin{tabular}{|l|l|l|l|}
\hline MV & SV & Desvio & $\begin{array}{l}\text { Instantes de } \\
\text { Alteração } \\
\text { (minutos) }\end{array}$ \\
\hline Vazão de Refluxo $\left(\mathrm{u}_{1}\right)$ & $\begin{array}{l}\text { Composição na corrente } \\
\text { de fundo }\left(\mathrm{y}_{1}\right)\end{array}$ & $\begin{array}{l}+15 \%,-30 \% \text { e } \\
+15 \%\end{array}$ & 300,800 e 1600 \\
\hline $\begin{array}{l}\text { Vazão de Fluido de } \\
\text { Aquecimento }\left(\mathrm{u}_{2}\right)\end{array}$ & $\begin{array}{l}\text { Temperatura de topo } \\
\left(\mathrm{y}_{2}\right)\end{array}$ & $+5 \%,-10 \%$ e $+5 \%$ & 300,800 e 1600 \\
\hline
\end{tabular}

O resultado para a simulação de alteração do set-point para as variáveis controladas é apresentado Figura 6-1.
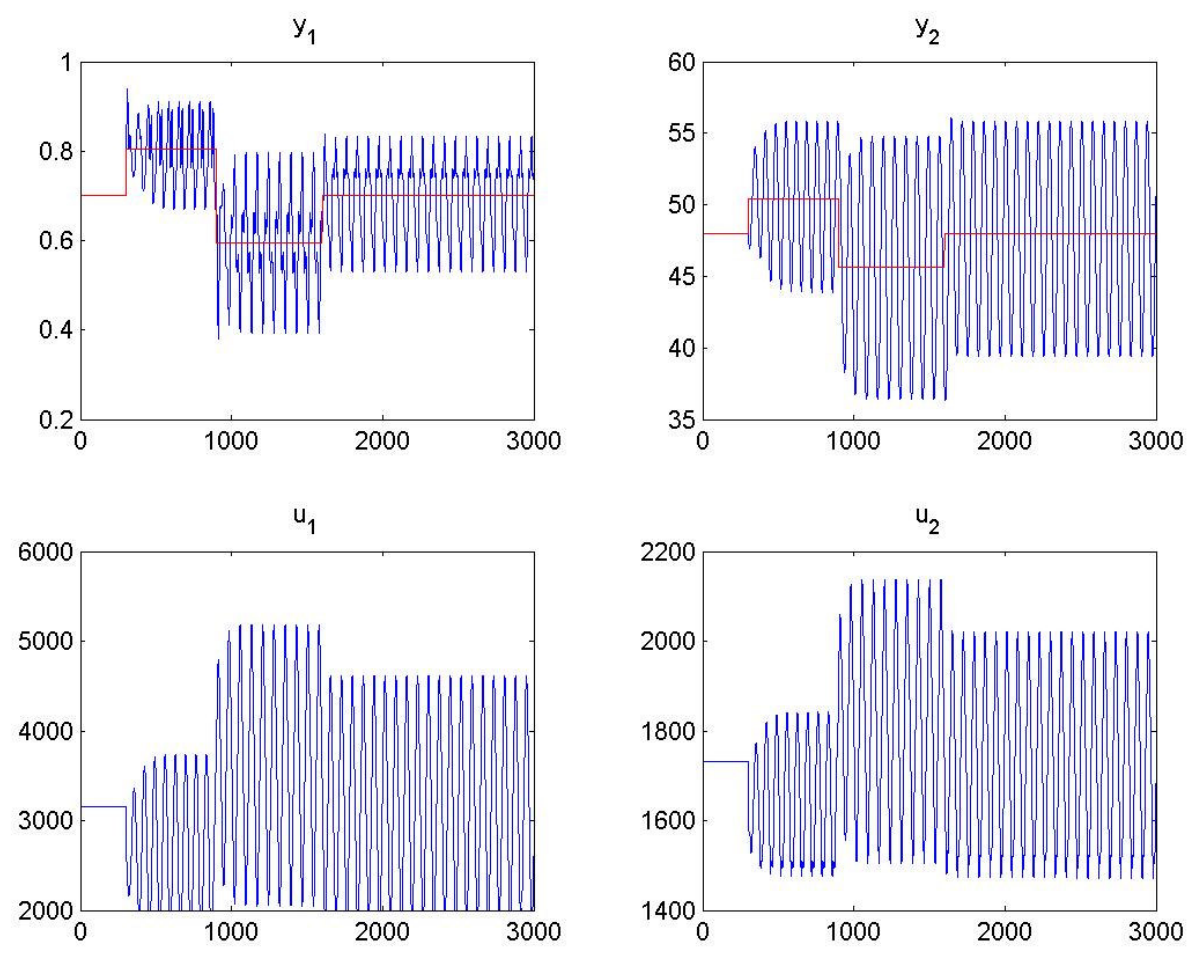

Figura 6-1 - Simulação - Planta: Modelo 1 x Controlador: Modelo FD 
Como pode ser verificado nesta simulação, o controlador com modelo FD não é capaz de controlar a planta representada pelo modelo 1, resultando comportamento oscilatório. Esta situação demonstra problemas que podem ser arremetidos ao modelo FD utilizado no controlador e, também, ao próprio algoritmo de controle o qual pode não ser o melhor para o processo em questão.

Em função de problemas com o modelo FD, Porfírio (2001) propôs o controlador MMPC com a identificação de outros modelos para representar diferentes pontos de operação conforme apresentado na Figura 4-2.

Assim, por ser uma condição não favorável à estabilidade do controlador, as simulações para o controlador AQDMC serão realizadas considerando o modelo FD como condição inicial para o controlador.

\subsection{Estudo dos Modelos}

Nesta seção será realizada a análise dos modelos presentes no Anexo A através de testes em degrau aplicado nas variáveis manipuladas dos modelos em questão. $O$ resultado desta análise é apresentado na Figura 6-2. 

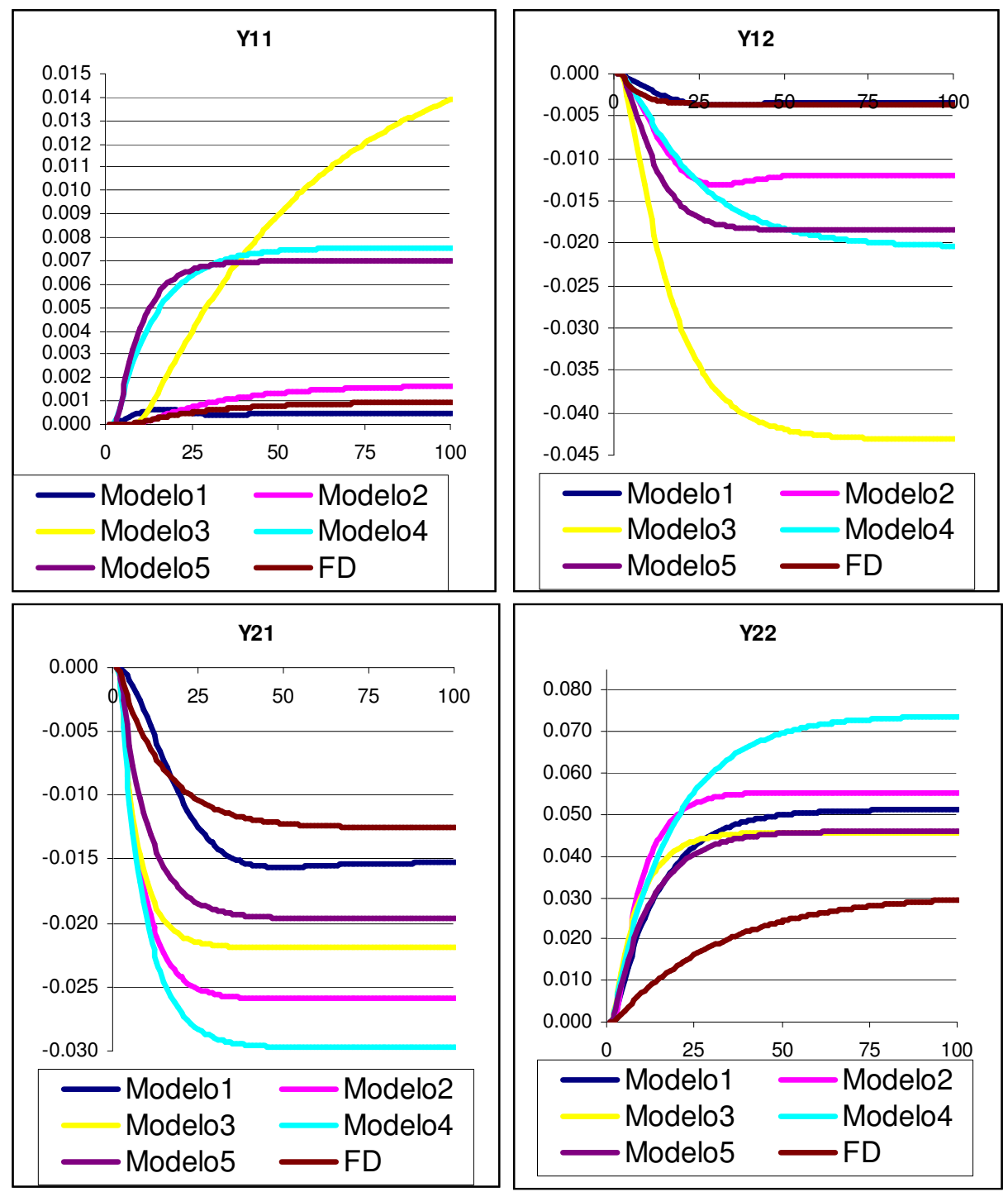

Figura 6-2 - Resposta ao Degrau - Modelos Porfírio (2001)

onde:

$\mathrm{y}_{11}$ - Concentração de $\mathrm{C}^{3-}$ na corrente de fundo x Vazão de refluxo

$\mathrm{y}_{12}$ - Concentração de $\mathrm{C}^{3-}$ na corrente de fundo $\mathrm{x}$ Vazão de fluido de aquecimento

$\mathrm{y}_{21}$ - Temperatura de Topo x Vazão de refluxo

$\mathrm{y}_{22}$ - Temperatura de Topo x Vazão de fluido de aquecimento

\subsubsection{Ganho dos Modelos}

Analisando a Figura 6-2, verifica-se que os modelos apresentam diferentes ganhos e diferentes dinâmicas. Por exemplo, para a variável $\mathrm{y}_{11}$, o ganho varia de 0.0005 até 0.015. Este comportamento é explicado pela diferença entre os pontos de operação 
utilizados na obtenção dos modelos como pode ser verificado na localização dos pontos de operação utilizado para levantar os modelos conforme apresentado na Figura 4-2.

\subsubsection{Tempo de Estabilização dos Modelos}

Novamente analisando a Figura 6-2 é possível verificar que o tempo de estabilização após a aplicação do teste degrau para a variável controla $y_{1}$ varia de 50 a 120 minutos. Enquanto que para a variável controlada - $y_{2}$, o tempo de estabilização varia de 40 a 80 minutos. Assim, $y_{1}$ apresenta ter uma dinâmica mais lenta quando comparado com $y_{2}$.

\subsubsection{Sinal de Excitação}

Conforme definido na seção 4.3.1, a excitação será realizada através de um sinal GBN. Para a aplicação deste sinal é necessário determinar o tempo médio decorrido entre duas alterações consecutivas $\left(E T_{S W}^{*}\right)$ através da equação (4.9) que depende do tempo de estabilização para cada uma das variáveis controladas. Todas as definições de tempo estão apresentadas na Tabela 6-4.

Tabela 6-4 - Parâmetros para definição do sinal GBN

\begin{tabular}{|l|l|l|}
\hline $\begin{array}{l}\text { Variável } \\
\text { Controlada }\end{array}$ & $\begin{array}{l}T_{s s} \\
\text { (minutos) }\end{array}$ & $\begin{array}{l}E T_{S W}^{*} \\
\text { (minutos) }\end{array}$ \\
\hline 1 & 120 & 39 \\
\hline 2 & 80 & 26 \\
\hline
\end{tabular}

Utilizando estes tempos e rotina desenvolvida em MatLab TM obtêm-se o perfil de excitação (+1 e -1) através dos sinais GBN o qual está representado na Figura 6-3 para uma simulação de 3 mil minutos. 


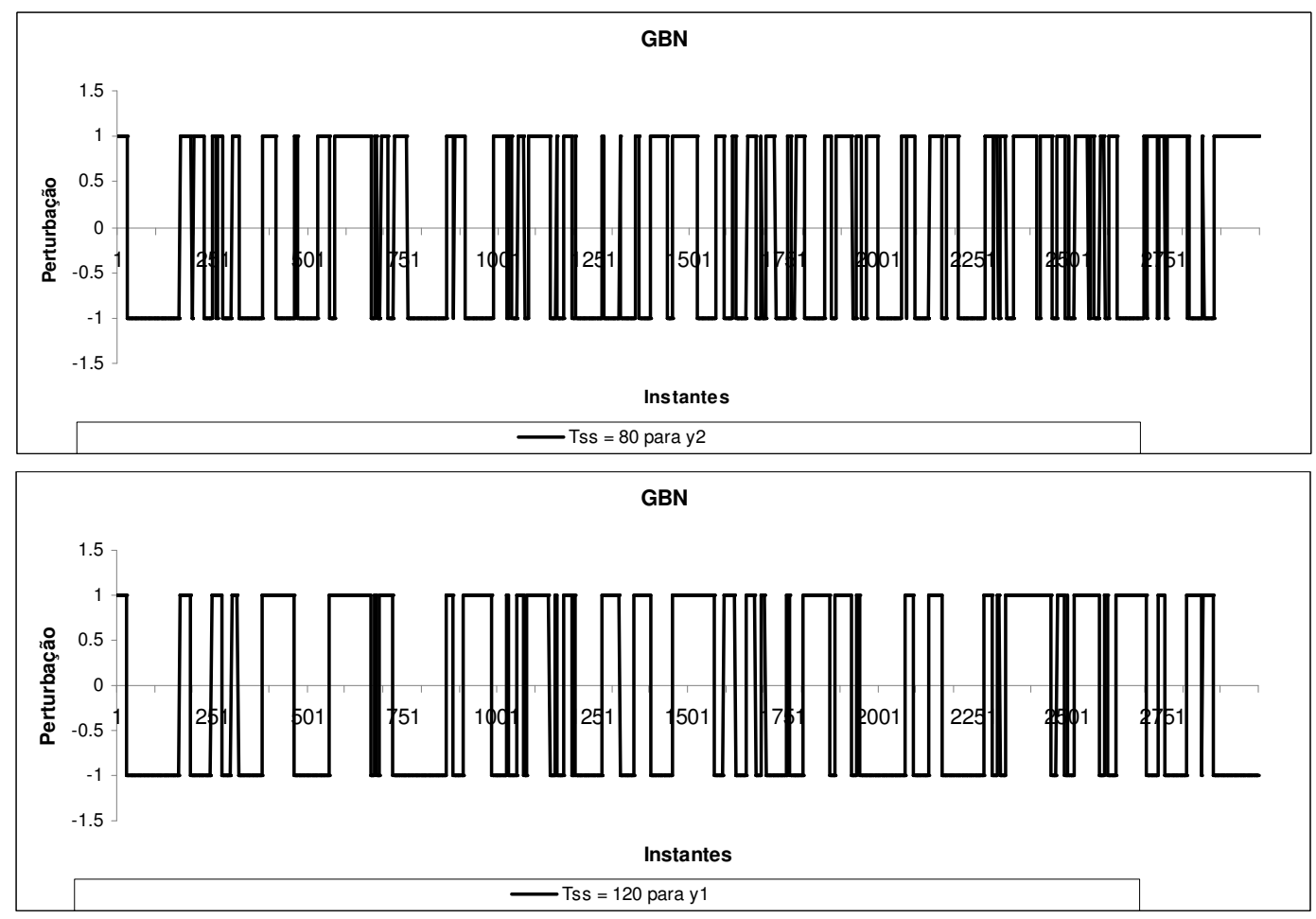

Figura 6-3 - Resposta característica para perturbação GBN

Uma vez determinada a seqüência GBN, é possível implementar a excitação através da variável $\mathrm{W}_{\text {exc }}$ a qual implicará a perturbação na camada de otimização do controlador adaptativo através do problema de otimização definido na seção 4.3.2 referente à Camada de Otimização.

\subsection{Análise Parâmetros do Controlador AQDMC}

Para que se possa implantar o controlador adaptativo é necessário enfocar três aspectos - tempo de identificação para determinar os coeficientes $\lambda_{i}\left(T_{\text {ident }}\right)$, forma de apresentação dos dados e tempo do experimento para realizar a excitação $\left(T_{\text {Teste }}\right)$.

\subsubsection{Tempo de Identificação - $T_{\text {ident }}$}

Para definir o valor ideal para $T_{\text {ident }}$ está sendo considerado $T_{\text {ident }}$ começando no valor 100 indo até 1000 com passo de 100 minutos. Sendo que em cada passo, 
deve ser resolvido o problema de otimização para a determinação do conjunto de $\lambda_{i}$ conforme apresentado na seção 4.2 referente ao Controlador Adaptativo.

Primeiramente, o teste é realizado considerando que a planta corresponde ao modelo 1 e o controlador utiliza o modelo FD na condição inicial. Assim, o modelo do controlador deve se aproximar do modelo $1\left(\lambda_{1}=1\right)$ ao longo dos instantes de simulação. O teste é repetido para a planta correspondente ao modelo 4. Assim, o modelo do controlador deve se aproximar do modelo $4\left(\lambda_{4}=1\right)$. O resultado está apresentado na Figura 6-4.

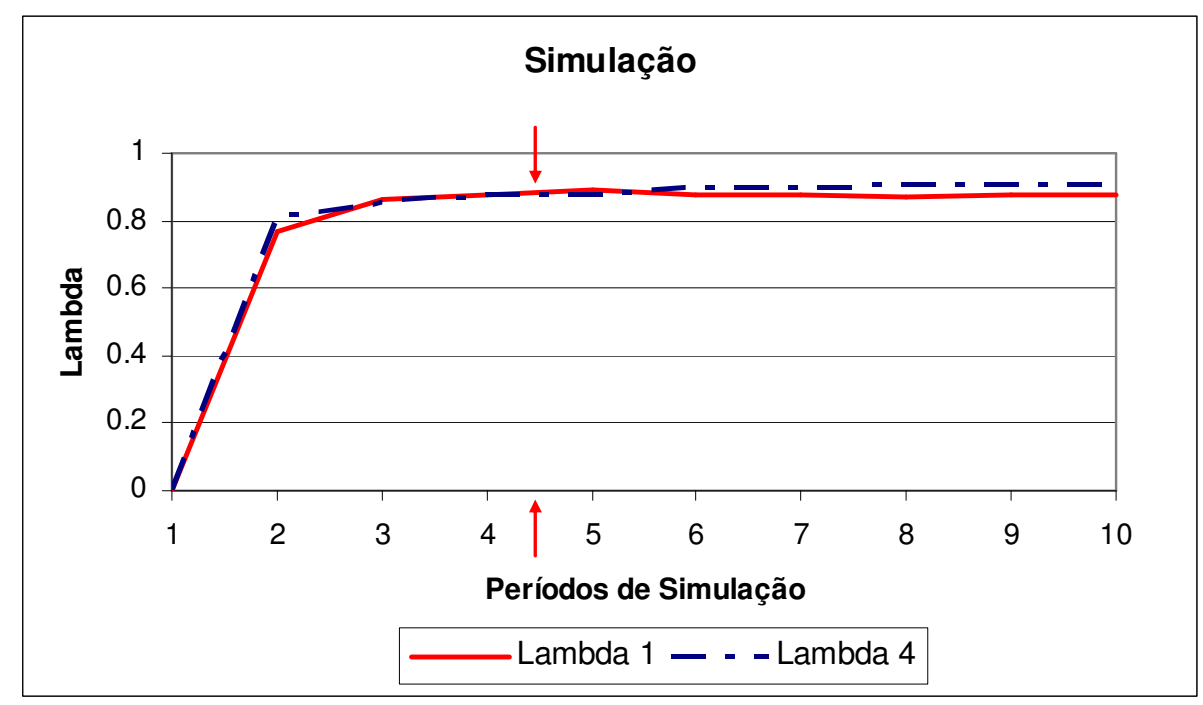

Figura 6-4 - Simulação para diferentes valores de $T_{\text {ident }}$

Analisando o resultado obtido acima, verifica-se que tanto o coeficiente $\lambda_{1}$ quanto 0 $\lambda_{4}$, não se alteram muito mais a partir do período 4.5. Assim, $T_{\text {ident }}$ é igual a $4.5 \times 100=450$ minutos.

\subsubsection{Forma de Apresentação dos Dados}

Neste caso não se trata de um parâmetro e sim de um procedimento. Basicamente, são duas abordagens a serem tratadas - considerando ou não uma janela de identificação móvel. Assim, o teste 1 foi realizado considerando ausência de uma janela de identificação móvel enquanto o teste 2 foi realizado com a janela de identificação móvel. 
Os parâmetros de sintonia do controlador são os mesmos apresentados na Tabela 6-1 com exceção aos parâmetros $y_{\min }$ e $y_{\max }$ os quais são definidos como sendo $y_{\min }=[0.5048]^{T}$ e $y_{\max }=[1.0050]^{T}$.

Enquanto que os parâmetros de sintonia para a camada PL serão os mesmos conforme apresentado na Tabela 6-2.

\subsubsection{Teste 1}

Neste caso, serão utilizados os dados correspondentes ao primeiro $T_{\text {ident }}$ minutos para se obter o primeiro conjunto de coeficientes $\lambda_{i}$. Em seguida, o segundo conjunto de coeficientes $\lambda_{i}$ será obtido considerando os dados coletados nos próximos $T_{i d e n t}$ minutos os quais não foram utilizados na obtenção do primeiro conjunto dos coeficientes $\lambda_{i}$, e assim sucessivamente. O resultado para este teste pode ser visualizado através da Tabela 6-5.

Tabela 6-5 - Coeficientes $\lambda_{i}$ obtidos pelo Teste 1

\begin{tabular}{|l|l|l|l|l|l|l|l|l|}
\hline$\#$ & Instantes & $\lambda_{1}$ & $\lambda_{2}$ & $\lambda_{3}$ & $\lambda_{4}$ & $\lambda_{5}$ & $\lambda_{6}$ & FO \\
\hline 0 & -- & 0.00 & 0.00 & 0.00 & 0.00 & 0.00 & 1.00 & $0.00 \mathrm{E}+00$ \\
\hline 1 & 0 a $T_{\text {ident }}$ & 0.94 & 0.06 & 0.00 & 0.00 & 0.00 & 0.00 & $6.24 \mathrm{E}-04$ \\
\hline 2 & $T_{\text {ident }}$ a $2 . T_{\text {ident }}$ & 1.00 & 0.00 & 0.00 & 0.00 & 0.00 & 0.00 & $8.51 \mathrm{E}-06$ \\
\hline 3 & $2 . T_{\text {ident }}$ a $3 . T_{\text {ident }}$ & -- & -- & -- & -- & -- & -- & -- \\
\hline
\end{tabular}

Conforme apresentado na Tabela 6-5, é realizada a identificação dos modelos 1 e 2 os quais são substituídos no controlador tão logo são identificados pela metodologia proposta de controlador adaptativo.

\subsubsection{Teste 2}

Diferentemente, o teste 2, por apresentar janela de identificação variável, terá a identificação com a sobreposição entre os instantes utilizados para a identificação dos diferentes conjuntos dos $\lambda_{i}$ conforme pode ser visto na Tabela 6-6. 
Tabela 6-6-Coeficientes $\lambda_{i}$ obtidos pelo Teste 2

\begin{tabular}{|l|l|l|l|l|l|l|l|l|}
\hline$\#$ & Intantes & $\lambda_{1}$ & $\lambda_{2}$ & $\lambda_{3}$ & $\lambda_{4}$ & $\lambda_{5}$ & $\lambda_{6}$ & FO \\
\hline 0 & -- & 0.00 & 0.00 & 0.00 & 0.00 & 0.00 & 1.00 & $0.00 \mathrm{E}+00$ \\
\hline 1 & 0 a $T_{\text {ident }}$ & 0.94 & 0.06 & 0.00 & 0.00 & 0.00 & 0.00 & $6.24 \mathrm{E}-04$ \\
\hline 2 & $0.5 * T_{\text {ident }}$ a $1.5 * T_{\text {ident }}$ & 0.80 & 0.00 & 0.00 & 0.00 & 0.10 & 0.11 & $7.91 \mathrm{E}-05$ \\
\hline 3 & $T_{\text {ident }}$ a $2 * T_{\text {ident }}$ & 1.00 & 0.00 & 0.00 & 0.00 & 0.00 & 0.00 & $8.50 \mathrm{E}-06$ \\
\hline 4 & $1.5 * T_{\text {ident }}$ a $2.5 * T_{\text {ident }}$ & -- & -- & -- & -- & -- & -- & -- \\
\hline
\end{tabular}

Verifica-se que o primeiro conjunto de $\lambda_{i}$ considera os instantes de 0 a $T_{\text {ident }}$. Para a identificação do segundo conjunto de $\lambda_{i}$, são considerados os instantes de $0.5 * T_{i d e n t}$ a $1.5 * T_{\text {ident }}$, ou seja, estão sendo utilizados $0.5 * T_{\text {ident }}$ instantes já utilizados na obtenção do primeiro conjunto de $\lambda_{i}$ e $0.5 * T_{\text {ident }}$ referente a um novo conjunto de dados. Para a identificação do terceiro conjunto de $\lambda_{i}$, são considerados os instantes de $T_{\text {ident }}$ a $2 * T_{\text {ident }}$, ou seja, estão sendo utilizados $0.5 * T_{\text {ident }}$ instantes já utilizados na obtenção do segundo conjunto de $\lambda_{i}$ e $0.5^{*} T_{\text {ident }}$ referente a um novo conjunto de dados, e assim sucessivamente.

Assim como para o teste 1 , no teste 2, tão logo é identificado o modelo pela metodologia proposta, o mesmo é substituído no controlador.

\subsubsection{Análise Geral para a Forma de Apresentação dos Dados}

Tanto o modelo obtido no teste 1 quanto o modelo obtido no teste 2 se aproximaram do modelo da planta. Assim, será utilizada a metodologia apresentada para o teste 1, ou seja, com ausência de janela de identificação móvel por ser uma abordagem mais próxima do que será feito na implantação prática do método, onde a identificação será realizada de tempos em tempos. 


\subsubsection{Tempo do Experimento - $T_{\text {Teste }}$}

O parâmetro $T_{\text {Teste }}$ está relacionado com o número de instantes em que o processo estará sob a influência da perturbação inserida através do sinal GBN. Para a presente análise estão sendo consideradas duas diferentes abordagens conforme apresentado por Conner e Seborg (2004) e Sotomayor et al. (2008).

Segundo Conner e Seborg (2004), para alguns pacotes comerciais de MPC, a duração do experimento deve ser $T_{\text {teste }}=6 .\left(n_{u}+n_{d}\right) \cdot T_{\max }$, onde $n_{u}$ é o número de entradas, $n_{d}$ é o número de variáveis de distúrbios medidas, $T_{\max }$ é o tempo máximo de estabilização do processo. Assim, aplicando a expressão definida por Conner e Seborg (2004) e os tempos de estabilização apresentados na Tabela 6-4 é possível determinar os tempos de experimento conforme apresentados na Tabela 6-7.

Tabela 6-7 - Tempo de Experimento por Conner e Seborg (2004)

\begin{tabular}{|c|c|}
\hline $\begin{array}{c}\mathrm{y}_{1} \\
\text { (minutos) }\end{array}$ & $\begin{array}{c}\mathrm{y}_{2} \\
\text { (minutos) }\end{array}$ \\
\hline 2900 & 2000 \\
\hline
\end{tabular}

Em Sotomayor et al. (2008), verifica-se que o tempo de experimento utilizado foi de 4500 minutos sendo que 3000 minutos foram utilizados para identificar o novo modelo e 1500 minutos foram utilizados na validação do modelo obtidos.

Para o presente trabalho está sendo considerado que $T_{\text {Teste }}$ seja igual a 3000 minutos de tal forma que possa ser comparado com as abordagens apresentadas por Conner e Seborg (2004) e Sotomayor et al. (2008). Além disso, este valor é maior do que o tempo de identificação $T_{\text {ident }}$, garantido assim, a obtenção de pelo menos 6 conjuntos dos coeficientes $\lambda_{i}$ para a identificação da planta.

\subsection{Casos de Estudo}

Nesta seção serão apresentados os resultados obtidos com a implantação do controlador adaptativo AQDMC. Para tanto, serão realizadas as simulações considerando os modelos de 1 a 5 apresentados no Anexo A para que possam 
representar a planta enquanto que para o controlador, o modelo será obtido pela solução do problema definido em (4.7).

Os parâmetros de sintonia do controlador são os mesmos apresentados na seção 6.3.2 a qual se refere à Forma de Apresentação dos Dados para o controlador Adaptativo.

Como condição inicial considera-se que o controlador assume o modelo FD para os seus primeiros $T_{\text {ident }}$ minutos. Após isto, o controlador adaptativo irá atualizar o modelo do controlador a partir da obtenção dos coeficientes $\lambda_{i}$ resolvendo 0 problema de otimização definido na seção 4.2 referente ao Controlador Adaptativo.

Para cada um destes conjuntos de coeficientes $\lambda_{i}$ serão também determinados os autovalores de tal forma a verificar se o grau de excitação foi suficiente para se identificar um novo modelo para o controlador.

\subsubsection{Caso 1 - Planta com Modelo 1}

O resultado da simulação é apresentado na Figura 6-5 para planta correspondente ao modelo 1 . 

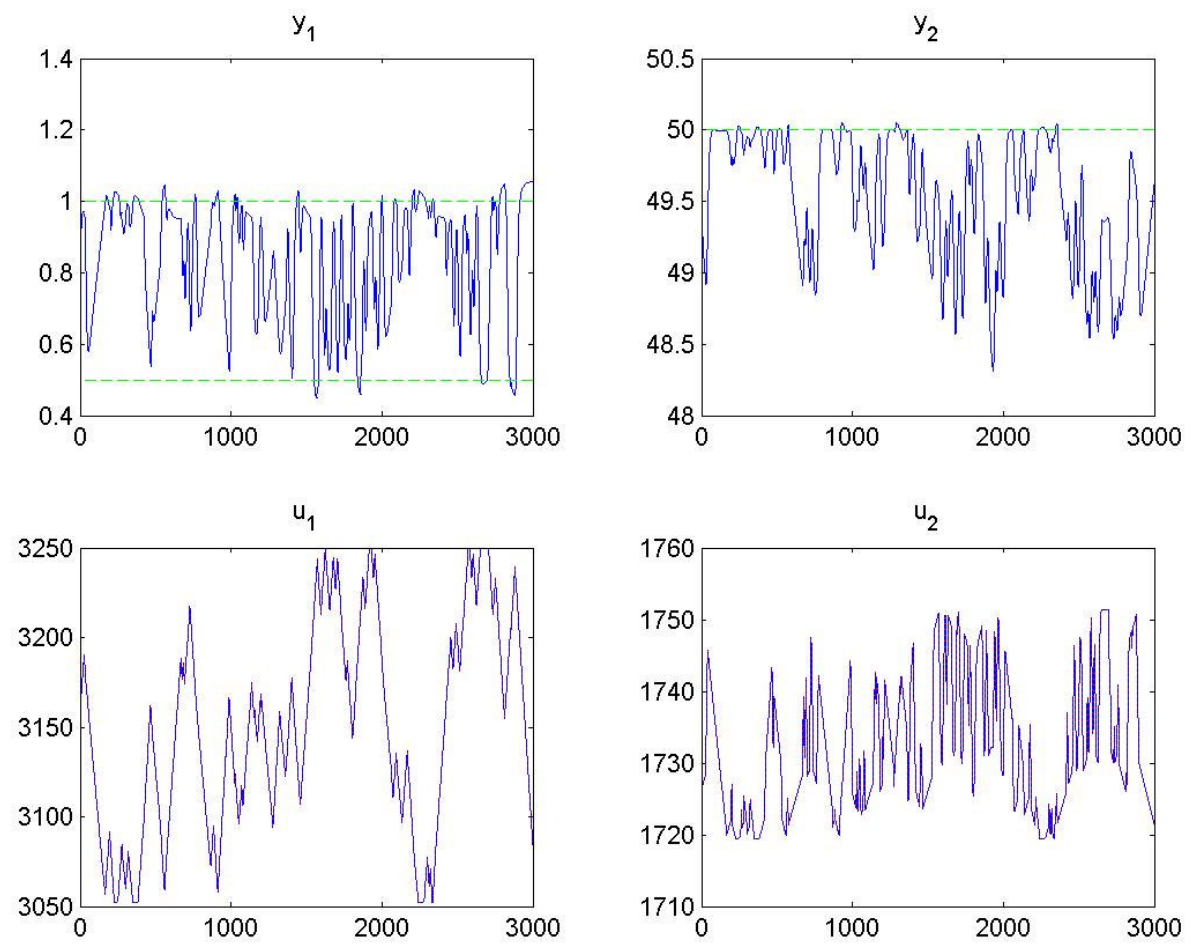

Figura 6-5 -Planta: Modelo 1 x Controlador: Modelo Combinado

Os conjuntos de coeficientes $\lambda_{i}$ obtidos ao longo de todo o experimento estão apresentados na Tabela 6-8.

Tabela 6-8 - Resultado da combinação entre os modelos

\begin{tabular}{|l|l|l|l|l|l|l|l|l|}
\hline$\#$ & $\lambda_{1}$ & $\lambda_{2}$ & $\lambda_{3}$ & $\lambda_{4}$ & $\lambda_{5}$ & $\lambda_{6}$ & Conv? & FO \\
\hline 0 & 0.00 & 0.00 & 0.00 & 0.00 & 0.00 & 1.00 & -- & -- \\
\hline 1 & 0.88 & 0.00 & 0.00 & 0.00 & 0.00 & 0.12 & $\operatorname{Sim}$ & $1.32 \mathrm{E}-04$ \\
\hline 2 & 1.00 & 0.00 & 0.00 & 0.00 & 0.00 & 0.00 & $\operatorname{Sim}$ & $1.01 \mathrm{E}-05$ \\
\hline 3 & 0.81 & 0.00 & 0.00 & 0.00 & 0.00 & 0.19 & $\operatorname{Sim}$ & $1.77 \mathrm{E}-04$ \\
\hline 4 & 0.90 & 0.00 & 0.04 & 0.00 & 0.01 & 0.04 & $\operatorname{Sim}$ & $5.17 \mathrm{E}-05$ \\
\hline 5 & 0.67 & 0.05 & 0.00 & 0.00 & 0.27 & 0.00 & $\operatorname{Sim}$ & $1.09 \mathrm{E}-03$ \\
\hline 6 & 1.00 & 0.00 & 0.00 & 0.00 & 0.00 & 0.00 & $\operatorname{Sim}$ & $8.50 \mathrm{E}-05$ \\
\hline
\end{tabular}

Onde o autovalor mínimo da matriz $\left(\bar{A}^{T} \cdot \bar{A}\right)$, para todos os conjuntos dos coeficientes de $\lambda_{i}$ está acima de 0.0041 . 


\subsubsection{Caso 2 - Planta com Modelo 2}

A próxima simulação é realizada considerando que o modelo da planta corresponde ao modelo 2 conforme se verifica pela Figura 6-6.
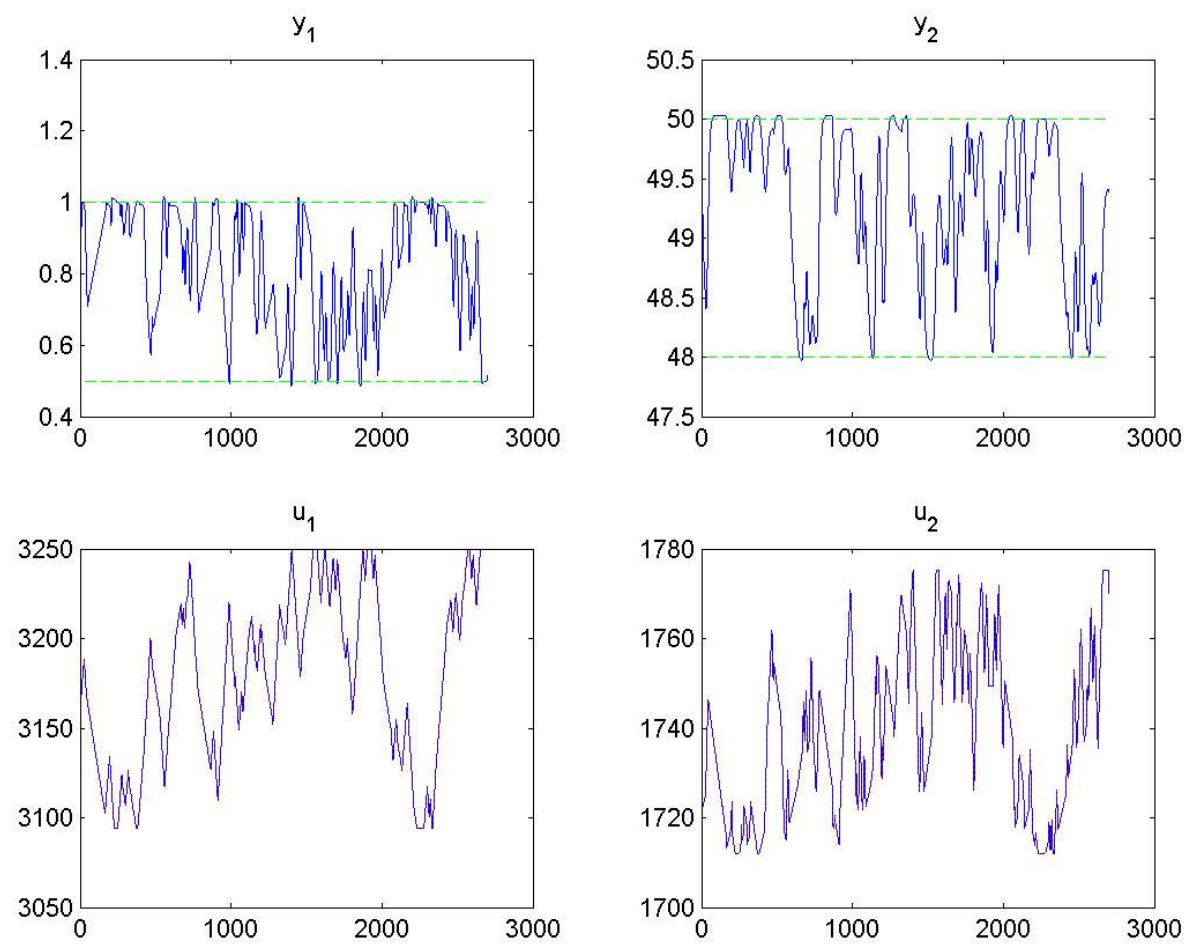

Figura 6-6 -Planta: Modelo 2 x Controlador: Modelo Combinado

Os conjuntos de coeficientes $\lambda_{i}$ obtidos ao longo de todo o experimento estão apresentados na Tabela 6-9.

Tabela 6-9 - Resultado da combinação entre os modelos

\begin{tabular}{|l|l|l|l|l|l|l|l|l|}
\hline$\#$ & $\lambda_{1}$ & $\lambda_{2}$ & $\lambda_{3}$ & $\lambda_{4}$ & $\lambda_{5}$ & $\lambda_{6}$ & Conv? & FO \\
\hline 0 & 0 & 0 & 0 & 0 & 0 & 1 & -- & -- \\
\hline 1 & 0.11 & 0.89 & 0.00 & 0.00 & 0.00 & 0.00 & Sim & $2.74 \mathrm{E}-04$ \\
\hline 2 & 0.00 & 0.91 & 0.01 & 0.07 & 0.00 & 0.00 & Sim & $2.59 \mathrm{E}-04$ \\
\hline 3 & 0.04 & 0.96 & 0.00 & 0.00 & 0.00 & 0.00 & Sim & $4.24 \mathrm{E}-04$ \\
\hline 4 & 0.00 & 1.00 & 0.00 & 0.00 & 0.00 & 0.00 & Sim & $1.05 \mathrm{E}-05$ \\
\hline 5 & 0.00 & 0.90 & 0.00 & 0.10 & 0.00 & 0.00 & Sim & $3.28 \mathrm{E}-03$ \\
\hline 6 & 0.12 & 0.88 & 0.00 & 0.00 & 0.00 & 0.00 & Sim & $4.48 \mathrm{E}-03$ \\
\hline
\end{tabular}


Onde o autovalor mínimo da matriz $\left(\bar{A}^{T} \cdot \bar{A}\right)$, para todos os conjuntos dos coeficientes de $\lambda_{i}$ está acima de 0.0048 .

\subsubsection{Caso 3 - Planta com Modelo 3}

O resultado da simulação é apresentado na Figura 6-7 para planta correspondente ao modelo 3.
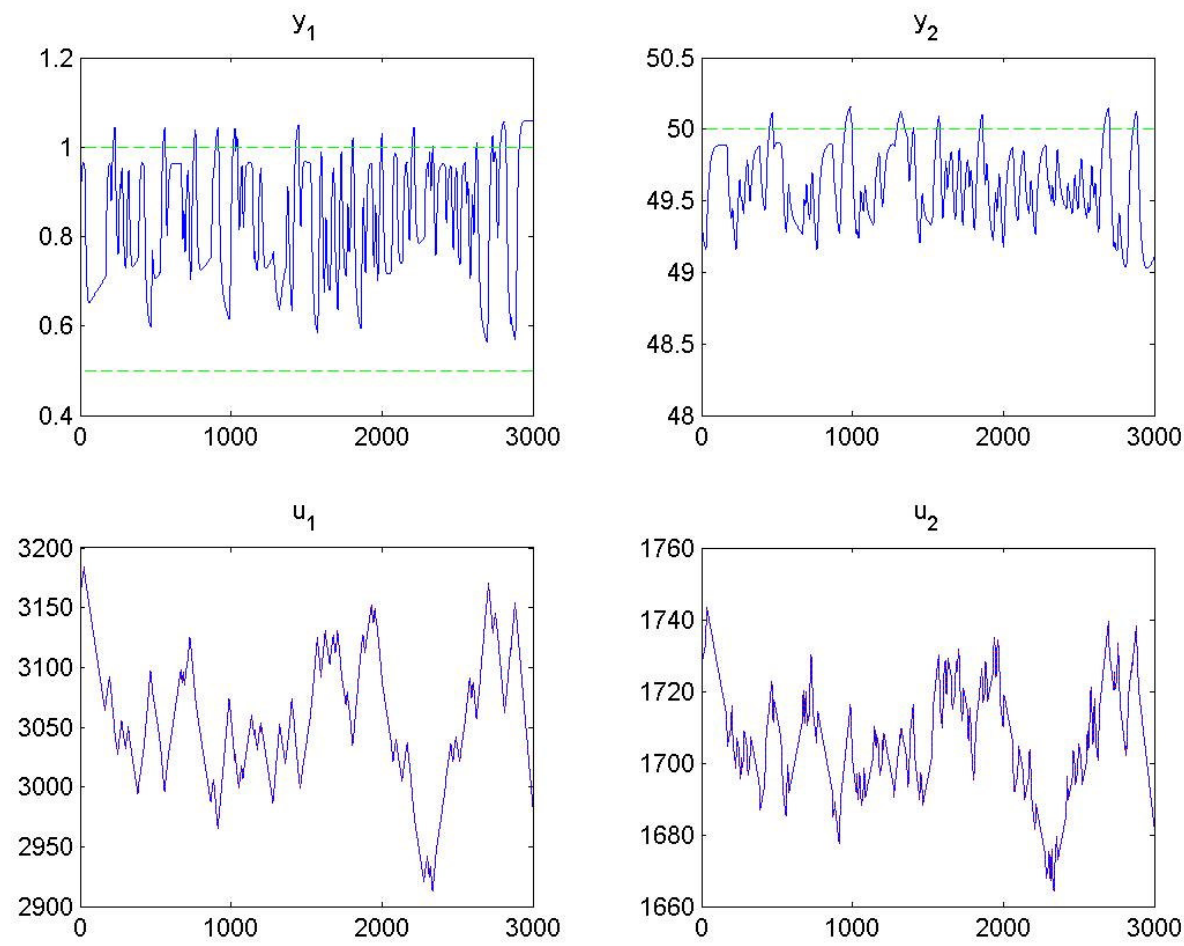

Figura 6-7 - Planta: Modelo 3 x Controlador: Modelo Combinado

Os conjuntos de coeficientes $\lambda_{i}$ obtidos ao longo de todo o experimento estão apresentados na Tabela 6-10. 
Tabela 6-10 - Resultado da combinação entre os modelos

\begin{tabular}{|l|l|l|l|l|l|l|l|l|}
\hline$\#$ & $\lambda_{1}$ & $\lambda_{2}$ & $\lambda_{3}$ & $\lambda_{4}$ & $\lambda_{5}$ & $\lambda_{6}$ & Conv? & FO \\
\hline 0 & 0.00 & 0.00 & 0.00 & 0.00 & 0.00 & 1.00 & -- & -- \\
\hline 1 & 0.01 & 0.02 & 0.73 & 0.07 & 0.05 & 0.12 & Sim & $5.41 \mathrm{E}-05$ \\
\hline 2 & 0.07 & 0.00 & 0.93 & 0.00 & 0.00 & 0.00 & Sim & $9.08 \mathrm{E}-06$ \\
\hline 3 & 0.09 & 0.01 & 0.88 & 0.02 & 0.00 & 0.00 & Sim & $1.82 \mathrm{E}-05$ \\
\hline 4 & 0.04 & 0.00 & 0.90 & 0.04 & 0.00 & 0.01 & Sim & $3.76 \mathrm{E}-05$ \\
\hline 5 & 0.04 & 0.02 & 0.67 & 0.14 & 0.12 & 0.00 & Sim & $2.10 \mathrm{E}-04$ \\
\hline 6 & 0.00 & 0.00 & 0.66 & 0.14 & 0.06 & 0.15 & $\operatorname{Sim}$ & $1.49 \mathrm{E}-04$ \\
\hline
\end{tabular}

Onde o autovalor mínimo da matriz $\left(\bar{A}^{T} \cdot \bar{A}\right)$, para todos os conjuntos dos coeficientes de $\lambda_{i}$ está acima de 0.072 .

\subsubsection{Caso 4 - Planta com Modelo 4}

Uma nova simulação é realizada considerando que a planta corresponde ao modelo 4 conforme está sendo apresentado na Figura 6-8.
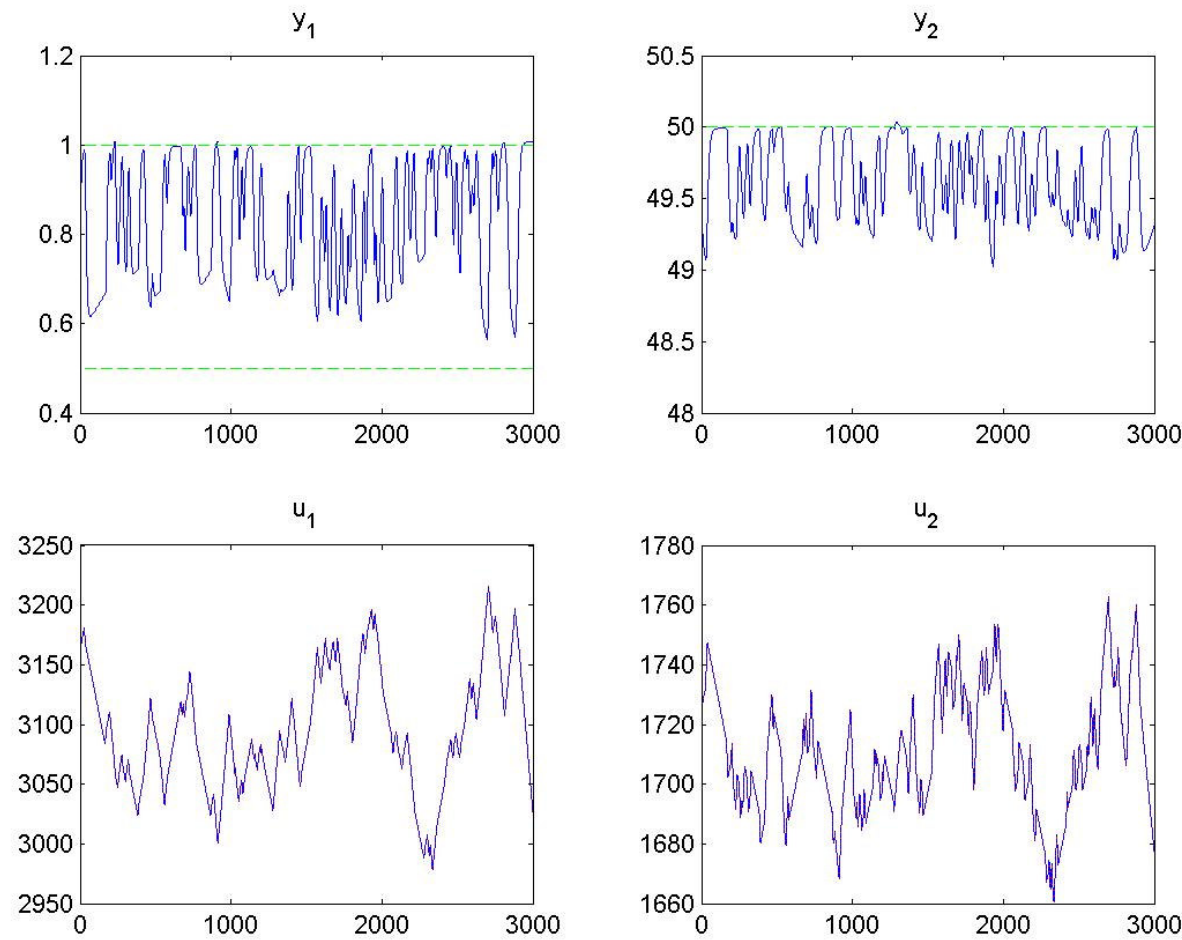

Figura 6-8 - Planta: Modelo 4 x Controlador: Modelo Combinado 
Os conjuntos de coeficientes $\lambda_{i}$ obtidos ao longo de todo o experimento estão apresentados na Tabela 6-11.

Tabela 6-11 - Resultado da combinação entre os modelos

\begin{tabular}{|l|l|l|l|l|l|l|l|l|}
\hline$\#$ & $\lambda_{1}$ & $\lambda_{2}$ & $\lambda_{3}$ & $\lambda_{4}$ & $\lambda_{5}$ & $\lambda_{6}$ & Conv? & FO \\
\hline 0 & 0.00 & 0.00 & 0.00 & 0.00 & 0.00 & 1.00 & -- & -- \\
\hline 1 & 0.00 & 0.01 & 0.00 & 0.89 & 0.10 & 0.00 & Sim & $7.95 \mathrm{E}-05$ \\
\hline 2 & 0.00 & 0.06 & 0.00 & 0.86 & 0.08 & 0.00 & Sim & $4.30 \mathrm{E}-04$ \\
\hline 3 & 0.00 & 0.00 & 0.00 & 0.99 & 0.00 & 0.01 & Sim & $3.24 \mathrm{E}-06$ \\
\hline 4 & 0.00 & 0.00 & 0.00 & 0.99 & 0.00 & 0.01 & Sim & $1.05 \mathrm{E}-05$ \\
\hline 5 & 0.00 & 0.18 & 0.00 & 0.63 & 0.19 & 0.00 & Sim & $3.80 \mathrm{E}-03$ \\
\hline 6 & 0.00 & 0.00 & 0.00 & 0.99 & 0.01 & 0.00 & Sim & $7.84 \mathrm{E}-06$ \\
\hline
\end{tabular}

Onde o autovalor mínimo da matriz $\left(\bar{A}^{T} \cdot \bar{A}\right)$, para todos os conjuntos dos coeficientes de $\lambda_{i}$ está acima de 0.0028 .

\subsubsection{Caso 5 - Planta com Modelo 5}

Finalmente, é realizada a simulação considerando que o modelo da planta corresponde ao modelo 5 conforme apresentado na Figura 6-9. 

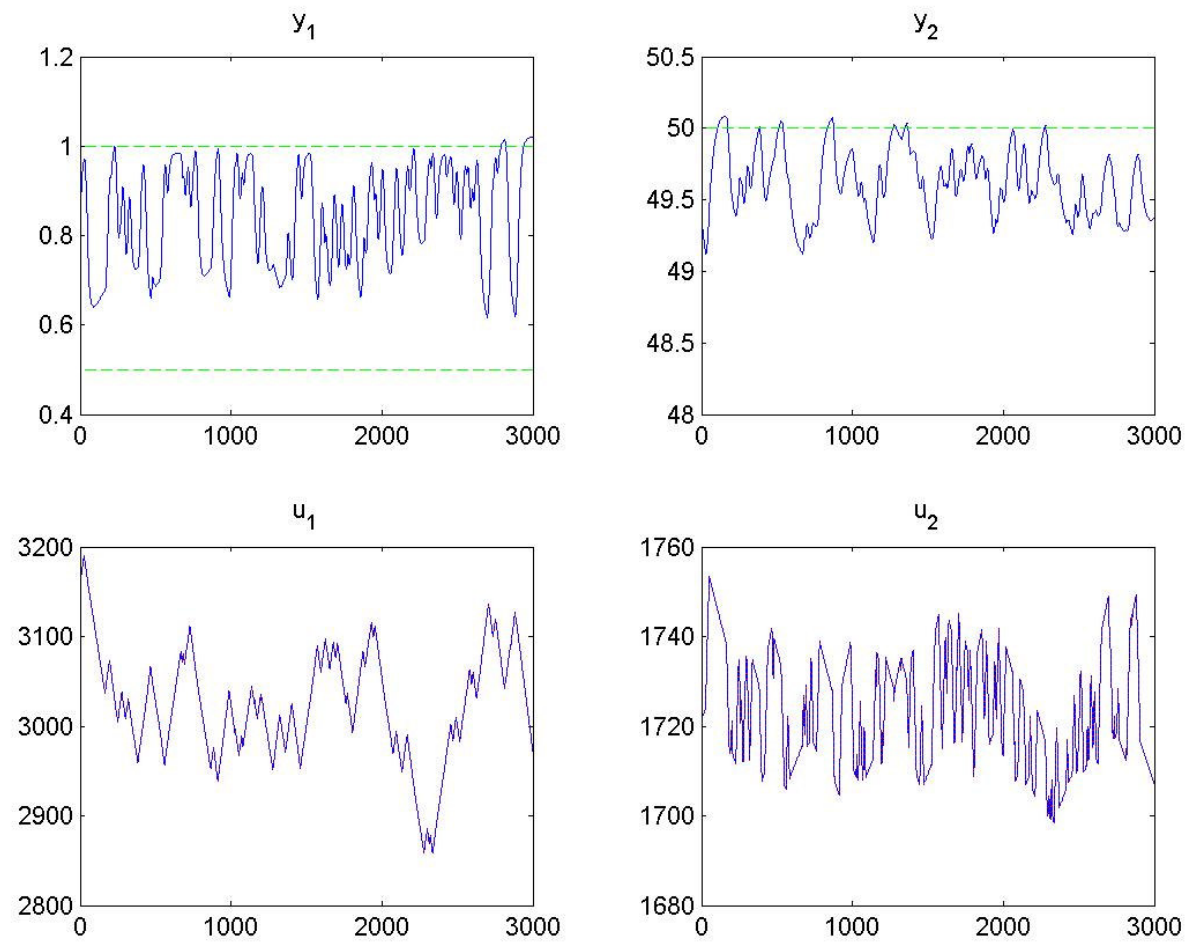

Figura 6-9 - Planta: Modelo 5 x Controlador: Modelo Combinado

Os conjuntos de coeficientes $\lambda_{i}$ obtidos ao longo de todo o experimento estão apresentados na Tabela 6-12.

Tabela 6-12 - Resultado da combinação entre os modelos

\begin{tabular}{|l|l|l|l|l|l|l|l|l|}
\hline$\#$ & $\lambda_{1}$ & $\lambda_{2}$ & $\lambda_{3}$ & $\lambda_{4}$ & $\lambda_{5}$ & $\lambda_{6}$ & Conv? & FO \\
\hline 0 & 0.00 & 0.00 & 0.00 & 0.00 & 0.00 & 1.00 & -- & -- \\
\hline 1 & 0.00 & 0.00 & 0.00 & 0.00 & 0.96 & 0.04 & Sim & $2.34 \mathrm{E}-04$ \\
\hline 2 & 0.00 & 0.04 & 0.00 & 0.00 & 0.96 & 0.00 & Sim & $1.11 \mathrm{E}-02$ \\
\hline 3 & 0.00 & 0.11 & 0.00 & 0.00 & 0.89 & 0.00 & Sim & $2.52 \mathrm{E}-03$ \\
\hline 4 & 0.00 & 0.00 & 0.01 & 0.00 & 0.99 & 0.00 & Sim & $4.05 \mathrm{E}-06$ \\
\hline 5 & 0.00 & 0.06 & 0.00 & 0.00 & 0.94 & 0.00 & Sim & $2.22 \mathrm{E}-02$ \\
\hline 6 & 0.00 & 0.00 & 0.00 & 0.00 & 1.00 & 0.00 & Sim & $1.47 \mathrm{E}-03$ \\
\hline
\end{tabular}

Onde o autovalor mínimo da matriz $\left(\bar{A}^{T} \cdot \bar{A}\right)$, para todos os conjuntos dos coeficientes de $\lambda_{i}$ está acima de 0.0011 . 


\subsubsection{Análise Geral dos Casos de Estudo}

Ao analisar as simulações presentes nas seções 6.4.1 a 6.4.5, verifica-se que os melhores modelos do controlador AQDMC identificado estavam muito próximos do modelo da planta quando a planta era igual aos modelos 1, 2, 4 e 5. Enquanto que para a planta 3, o melhor modelo do controlador identificado ficou um pouco afastado do modelo da planta.

Ao analisar os autovalores obtidos com a aplicação da metodologia apresentada na seção 4.4 referente à Caracterização da Excitação Persistente, tem-se que são todos diferentes de zero, assim, a excitação empregada pode ser caracterizada como suficiente.

\subsection{Planta Variando entre os Modelos 1 a 5}

Para esta seção, a metodologia do controlador adaptativo está sendo testada considerando que o modelo da planta está variando ao longo da simulação a cada 2250 minutos.

O resultado da simulação é apresentado na Figura 6-10 considerando 11500 minutos de simulação e considerando a existência dos 5 modelos. 

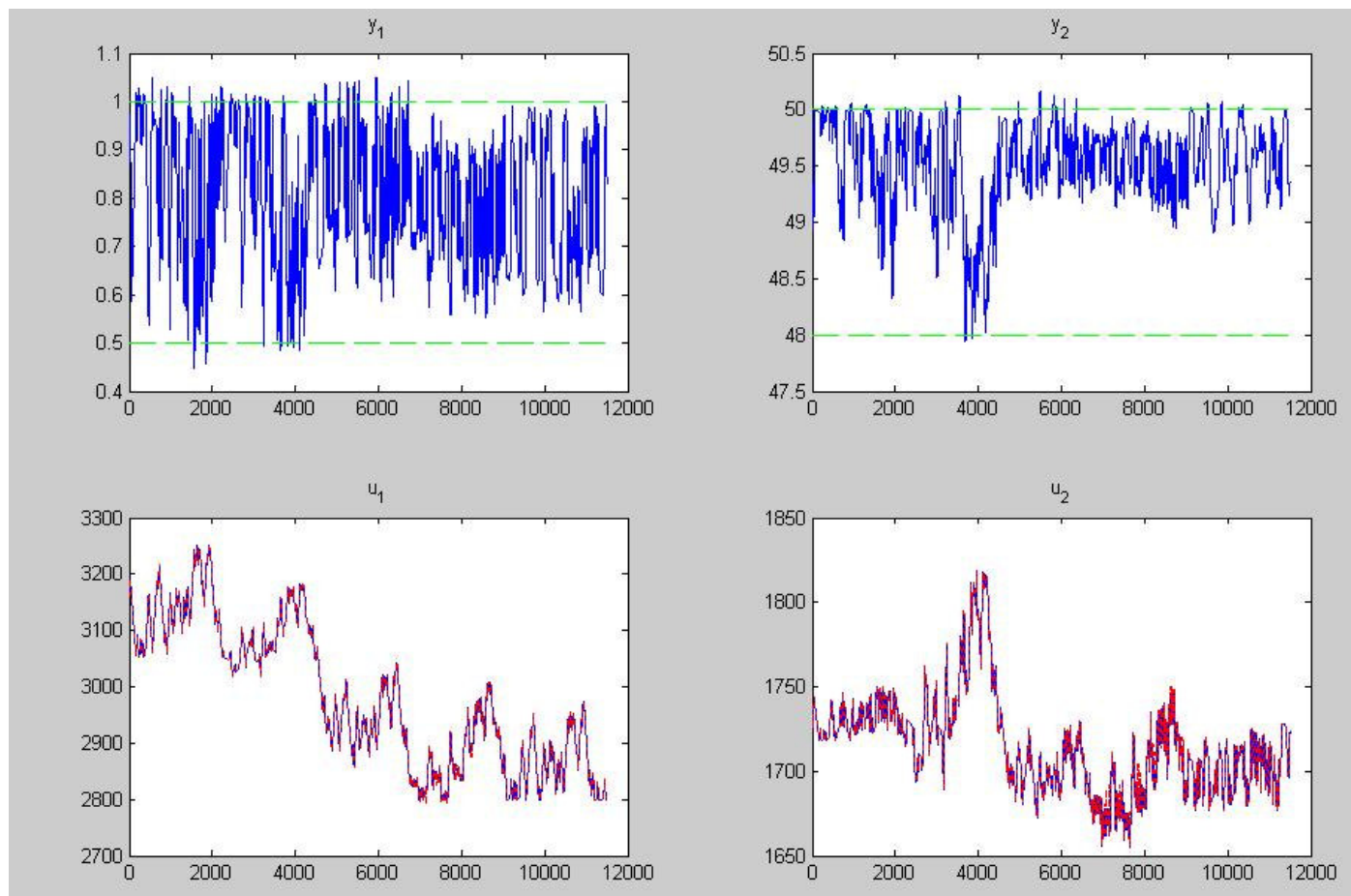

Figura 6-10 - Planta: Modelos 1 a 5 x Controlador: Modelo Combinado

A ordem de alteração do modelo da planta e os instantes onde foi identificado o modelo e implementado no controlador, estão apresentados na Figura 6-11.

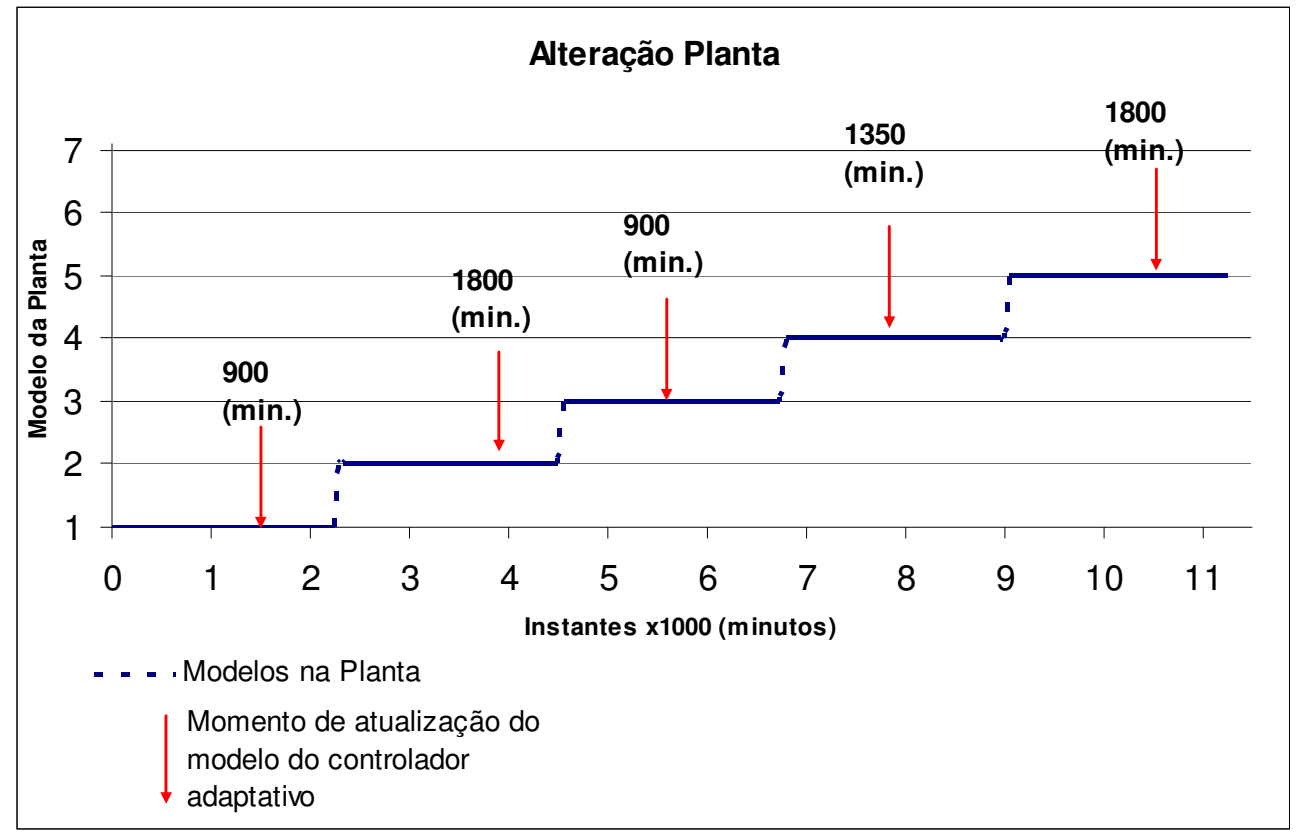

Figura 6-11 - Perfil de alteração dos modelos na planta

Os conjuntos dos coeficientes $\lambda_{i}$ que mais se aproximam do modelo da planta e os instantes de identificação estão apresentados na Tabela 6-13. 
Tabela 6-13 - Modelo do Controlador com Planta variando do Modelo 1 a 5

\begin{tabular}{|l|l|l|l|l|l|l|l|l|}
\hline Casos & $\begin{array}{l}\text { Instante } \\
(\mathrm{min} .)\end{array}$ & Planta & $\lambda_{1}$ & $\lambda_{2}$ & $\lambda_{3}$ & $\lambda_{4}$ & $\lambda_{5}$ & $\lambda_{6}$ \\
\hline 1 & 900 & Mod 1 & 1.00 & 0.00 & 0.00 & 0.00 & 0.00 & 0.00 \\
\hline 2 & 1800 & Mod 2 & 0.00 & 1.00 & 0.00 & 0.00 & 0.00 & 0.00 \\
\hline 3 & 900 & Mod 3 & 0.07 & 0.00 & 0.93 & 0.00 & 0.00 & 0.00 \\
\hline 4 & 1350 & Mod 4 & 0.00 & 0.00 & 0.00 & 0.99 & 0.01 & 0.00 \\
\hline 5 & 1800 & Mod 5 & 0.00 & 0.00 & 0.00 & 0.00 & 0.99 & 0.01 \\
\hline
\end{tabular}

Ao analisar os resultados obtidos com a alteração da planta verifica-se que os modelos identificados com a alteração da planta, ao longo da simulação, são idênticos aos modelos obtidos nas seções 6.4 .1 a 6.4 .5 onde a simulação era realizada considerando apenas um único modelo para a planta.

\subsection{Análise do Controlador Adaptativo}

Nesta seção será realizada a comparação do controlador AQDMC com o controlador MMPC, conforme apresentado na seção 6.6.1. Posteriormente, serão avaliados estes mesmos modelos em relação à metodologia de identificação através de um ARX conforme apresentado na seção 6.6.2.

Para todas estas comparações serão utilizados os modelos identificados que mais se aproximaram da planta conforme obtidos nas seções de 6.4.1 a 6.4.5 com a exceção de apenas um modelo o qual é o que mais se afasta da planta. Estes modelos estão resumidos na Tabela 6-14.

Tabela 6-14 - Validação dos modelos

\begin{tabular}{|l|l|l|l|l|l|l|l|}
\hline Caso & Planta & $\lambda_{1}$ & $\lambda_{2}$ & $\lambda_{3}$ & $\lambda_{4}$ & $\lambda_{5}$ & $\lambda_{6}$ \\
\hline 1 & Mod 1 & 1.00 & 0.00 & 0.00 & 0.00 & 0.00 & 0.00 \\
\hline 2 & Mod 2 & 0.00 & 1.00 & 0.00 & 0.00 & 0.00 & 0.00 \\
\hline 3 & Mod 3 & 0.07 & 0.00 & 0.93 & 0.00 & 0.00 & 0.00 \\
\hline 4 & Mod 4 & 0.00 & 0.00 & 0.00 & 0.99 & 0.00 & 0.01 \\
\hline 5 & Mod 4 & 0.00 & 0.18 & 0.00 & 0.63 & 0.19 & 0.00 \\
\hline 6 & Mod 5 & 0.00 & 0.00 & 0.01 & 0.00 & 0.99 & 0.00 \\
\hline
\end{tabular}


Conforme se verifica na Tabela 6-14, as combinações de número 1 a 4 e 6, são caracterizadas por estarem próximo do modelo da planta. Enquanto que a combinação de número 5 se afasta da planta.

\subsubsection{MMPC}

Nesta seção, os controladores usando modelos definidos na Tabela 6-14 serão comparados com o controlador MMPC que usa todos os modelos, simultaneamente, como apresentado na seção 5.1 .

Novamente, serão utilizados os mesmos parâmetros já apresentados na Tabela 6-1 com exceção dos novos valores para a abordagem do MMPC conforme definidos por Porfírio et al. (2003) e reproduzidos na Tabela 6-15.

Tabela 6-15 - Sintonia do Controlador MMPC

\begin{tabular}{|l|l|}
\hline Símbolo & Valor \\
\hline$n_{p}$ & 70 \\
\hline$R$ & $\operatorname{diag}\left[\begin{array}{ll}1.0 & 1.0\end{array}\right] \times 10^{-3}$ \\
\hline$Q$ & $\operatorname{diag}\left[\begin{array}{ll}1.05 & 1.0\end{array}\right]$ \\
\hline$y_{\max }$ & {$[0.7149]^{\top}$} \\
\hline$y_{\min }$ & {$[0.7048]^{\top}$} \\
\hline
\end{tabular}

Conforme definido na seção 5.1 durante a apresentação do controlador MMPC, a abordagem não considera os targets oriundos da camada estática da função objetivo. Assim, a presente simulação também não está levando em conta a presença da camada de otimização para o controlador MMPC.

Como forma de perturbar o sistema definido pelos controladores AQDMC e MMPC, será realizado o degrau nas faixas de controle para as variáveis controladas, no caso, $y_{\min }$ e $y_{\max }$. Os valores dos degraus estão apresentados na Tabela 6-16.

Tabela 6-16 - Desvio aplicados na simulação

\begin{tabular}{|l|l|l|}
\hline SV & Desvio & $\begin{array}{l}\text { Instantes de Alteração } \\
\text { (minutos) }\end{array}$ \\
\hline $\begin{array}{l}\text { Composição na corrente } \\
\text { de fundo }\left(\mathrm{y}_{1}\right)\end{array}$ & $+15 \%,-30 \%$ e $+15 \%$ & 300,800 e 2200 \\
\hline Temperatura de topo $\left(\mathrm{y}_{2}\right)$ & $+5 \%,-10 \%$ e $+5 \%$ & 300,800 e 2200 \\
\hline
\end{tabular}




\subsubsection{Caso 1 - Planta com modelo 1}

$\mathrm{Na}$

Figura 6-12 é apresentado o resultado do teste, tanto para AQDMC quanto para MMPC, considerando que o modelo da planta é representado pelo Modelo 1.

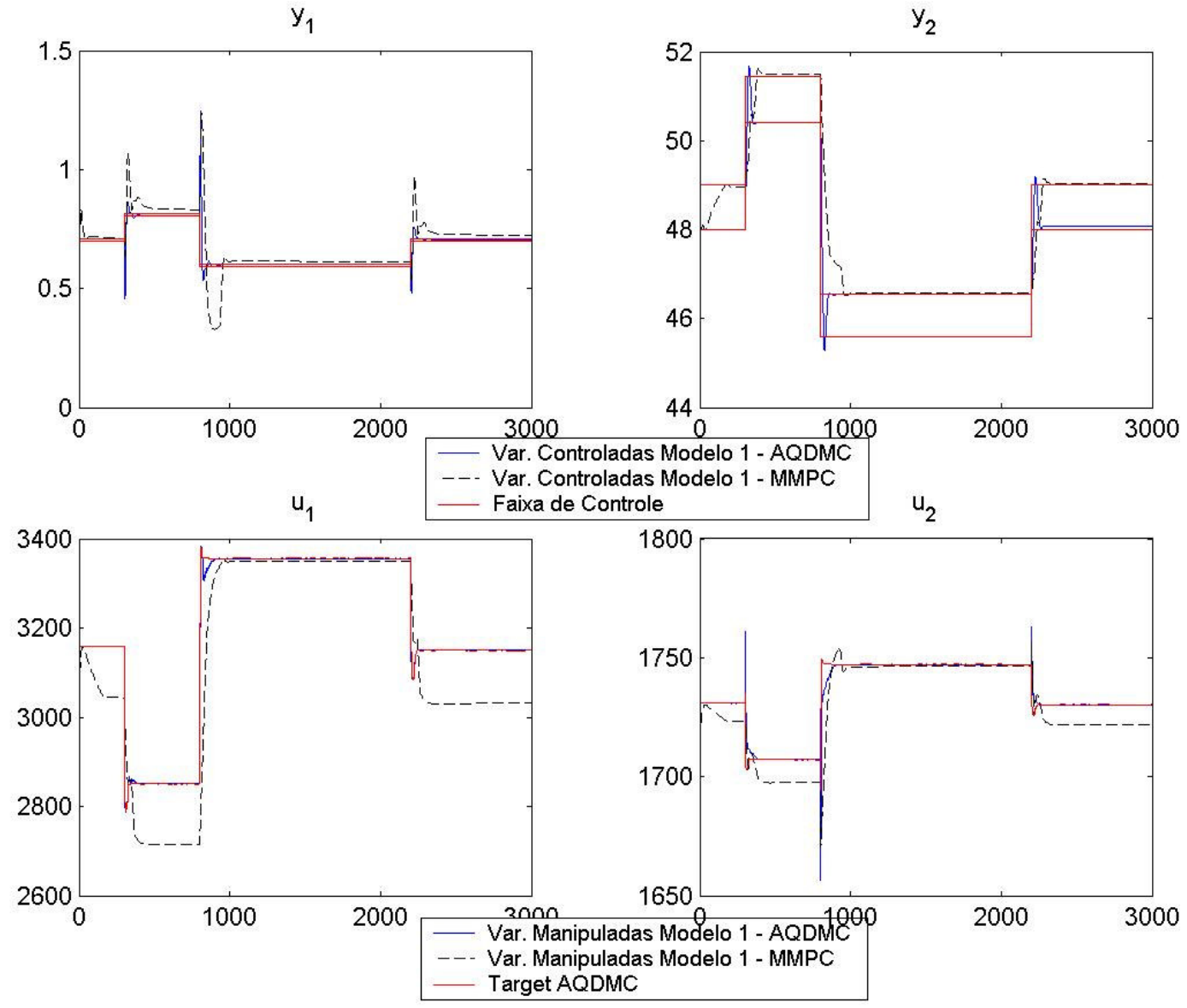

Figura 6-12 - Degrau - Planta: Modelo 1 x Controlador: Combinação entre os modelos

\subsubsection{Caso 2 - Planta com modelo 2}

Uma próxima simulação é realizada considerando que o modelo da planta corresponde ao modelo 2, tanto para o AQDMC quanto para o MMPC, conforme representado na Figura 6-13. 


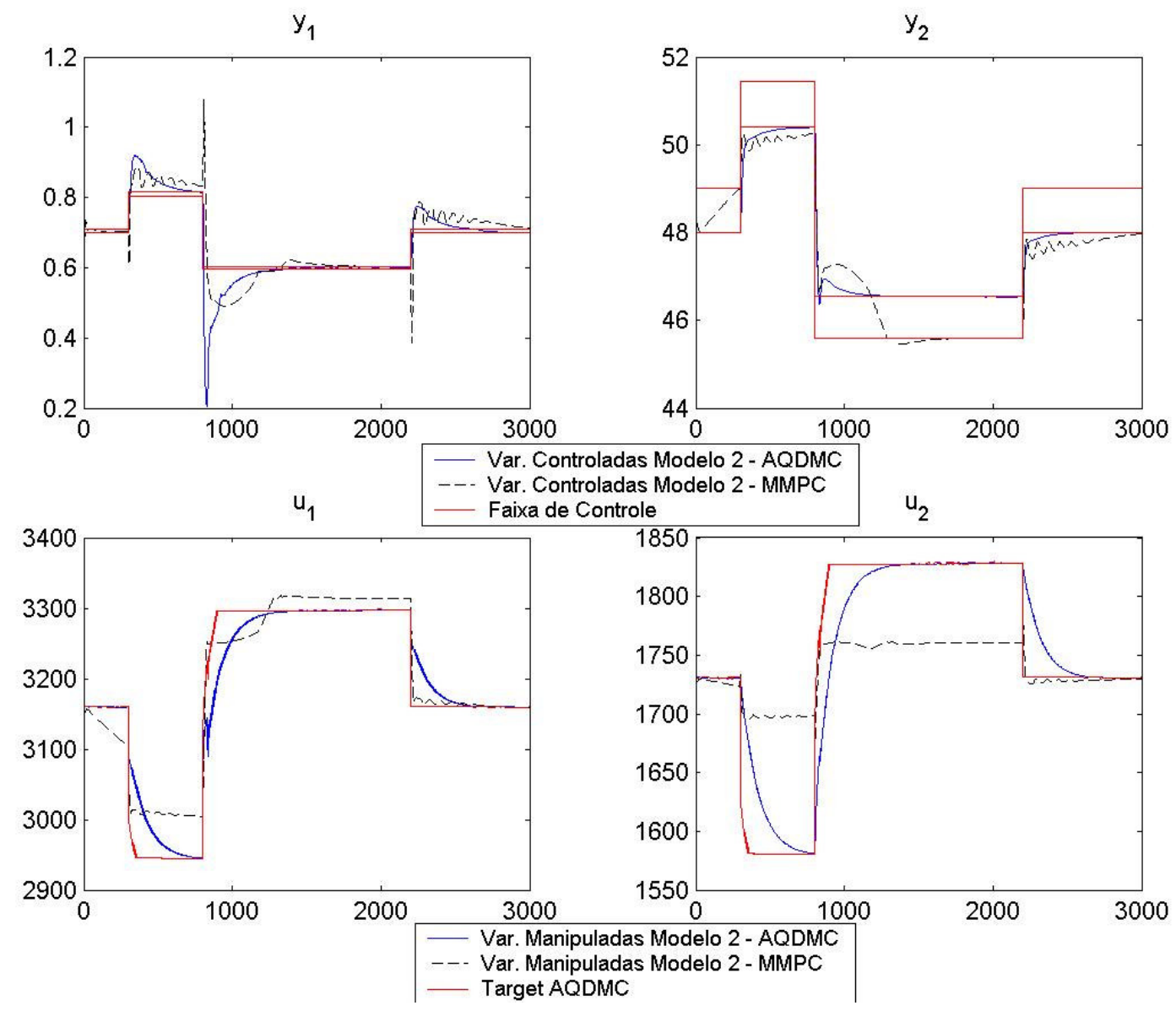

Figura 6-13 - Degrau - Planta: Modelo 2 x Controlador: Combinação entre os modelos

\subsubsection{Caso 3 - Planta com modelo 3}

Uma outra simulação é realizada considerando que o modelo da planta corresponde ao modelo 3 , tanto para o AQDMC quanto para o MMPC, conforme representado na

Figura 6-14. 


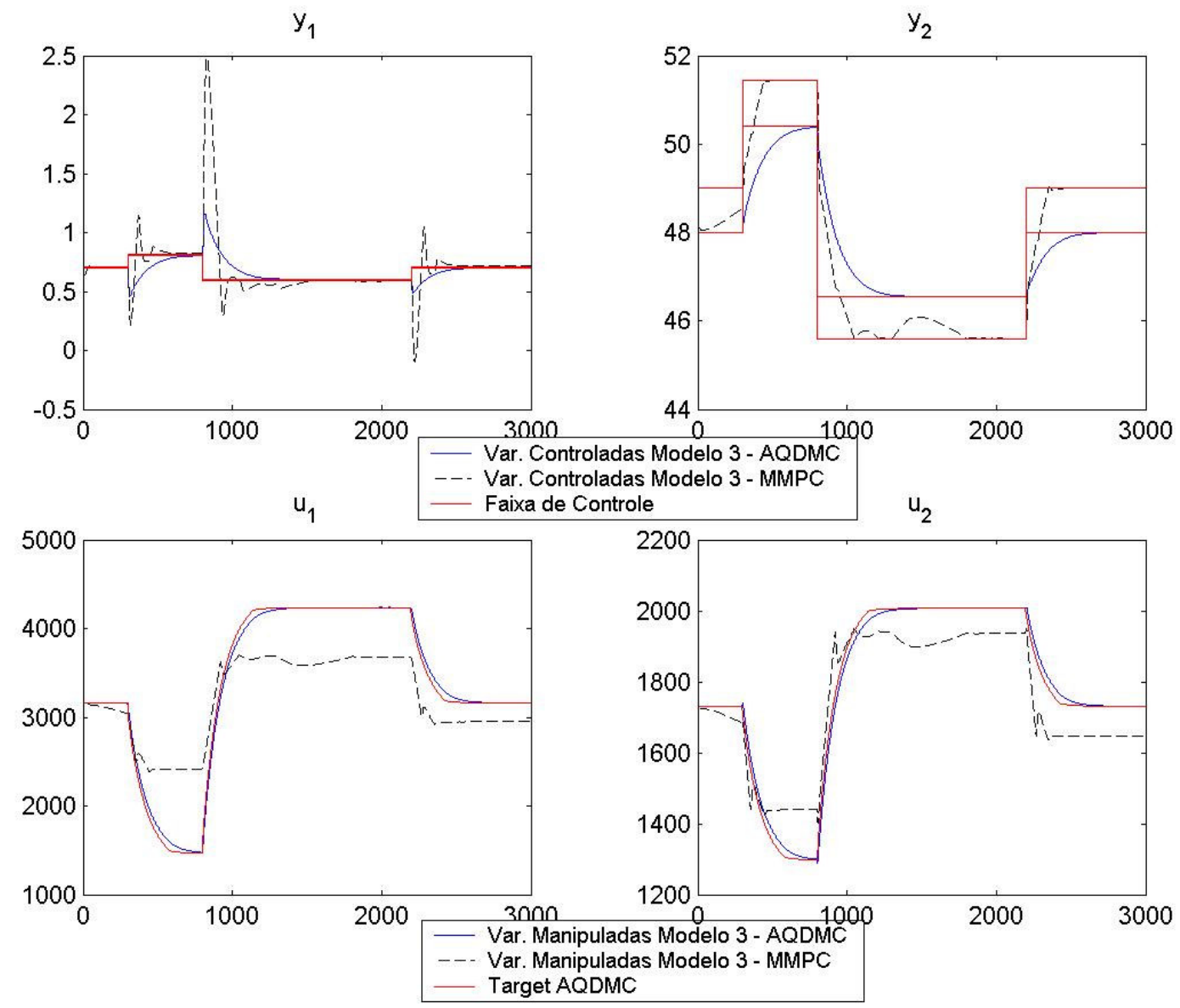

Figura 6-14 - Degrau - Planta: Modelo 3 x Controlador: Combinação entre os modelos

\subsubsection{Caso 4 - Planta com modelo 4}

$\mathrm{Na}$

Figura 6-15 é apresentado o resultado do teste, tanto para AQDMC quanto para MMPC, considerando que o modelo da planta é representado pelo Modelo 4. 


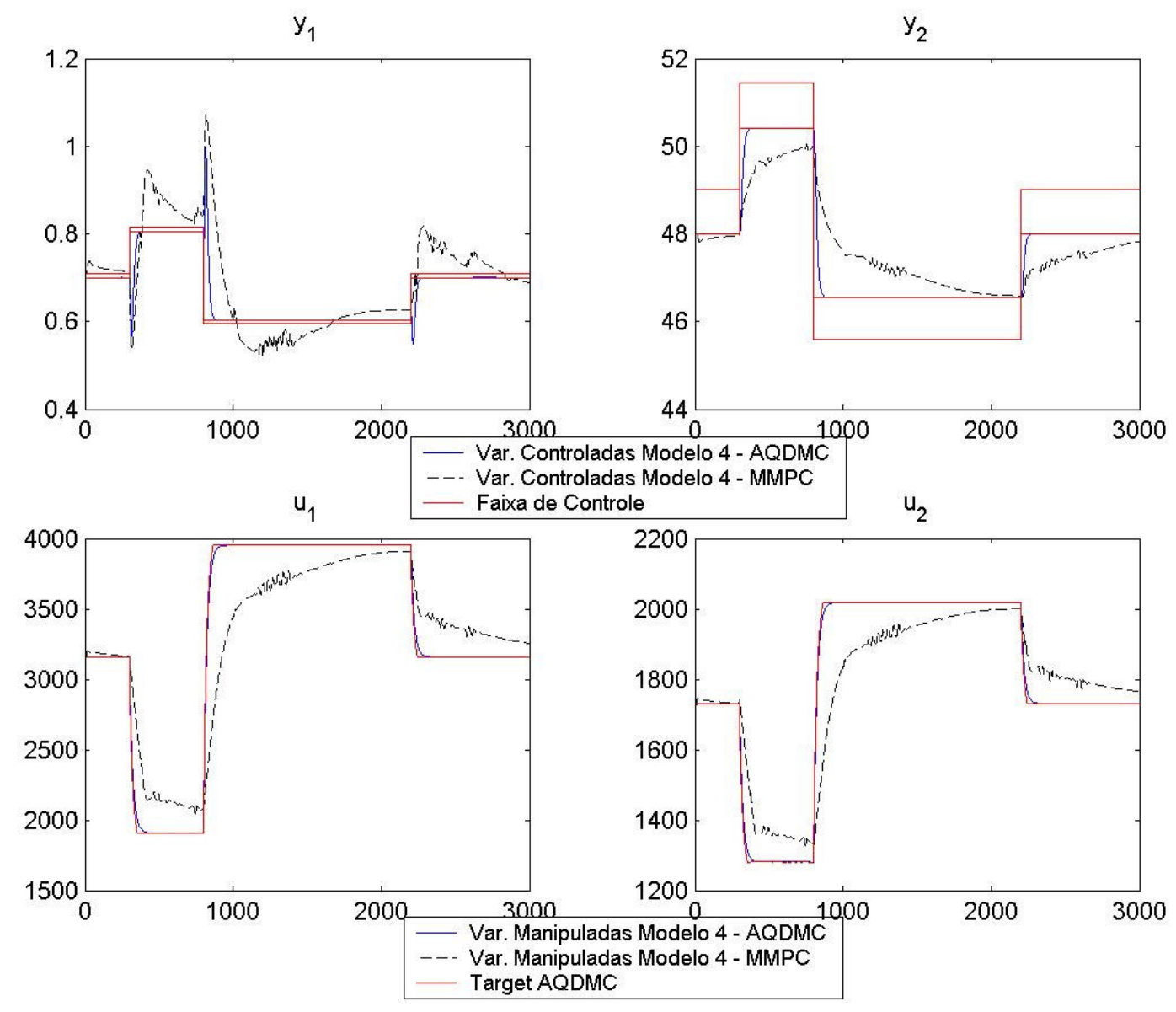

Figura 6-15 - Degrau - Planta: Modelo 4 x Controlador: Combinação entre os modelos 


\subsubsection{Caso 5 - Planta com modelo 4}

$\mathrm{Na}$

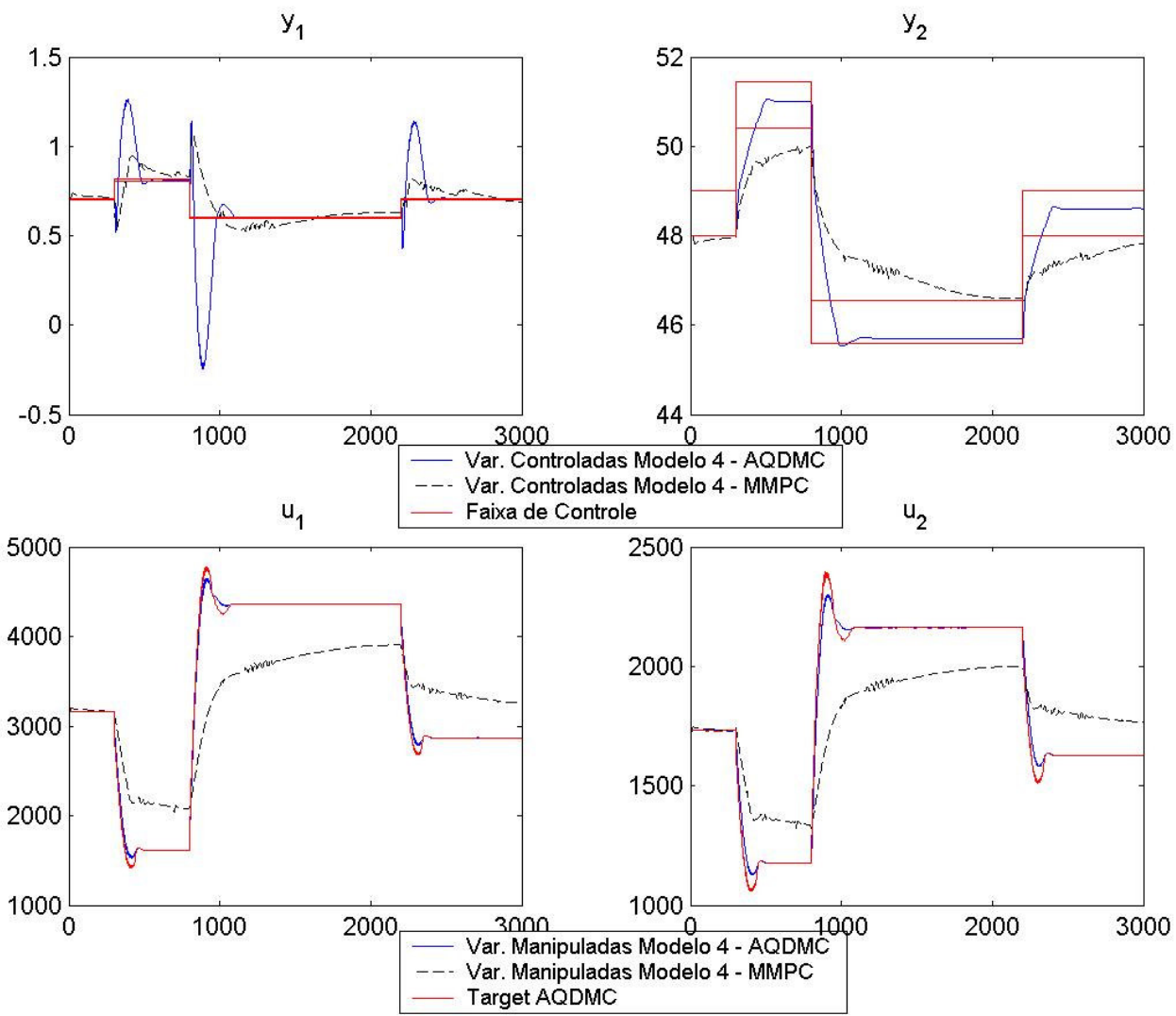

Figura 6-16 é apresentado o resultado do teste, tanto para AQDMC quanto para MMPC, considerando que o modelo da planta é representado pelo Modelo 4. Conforme é possível verificar na Tabela 6-14, o modelo do controlador adaptativo se afasta do modelo da planta. 


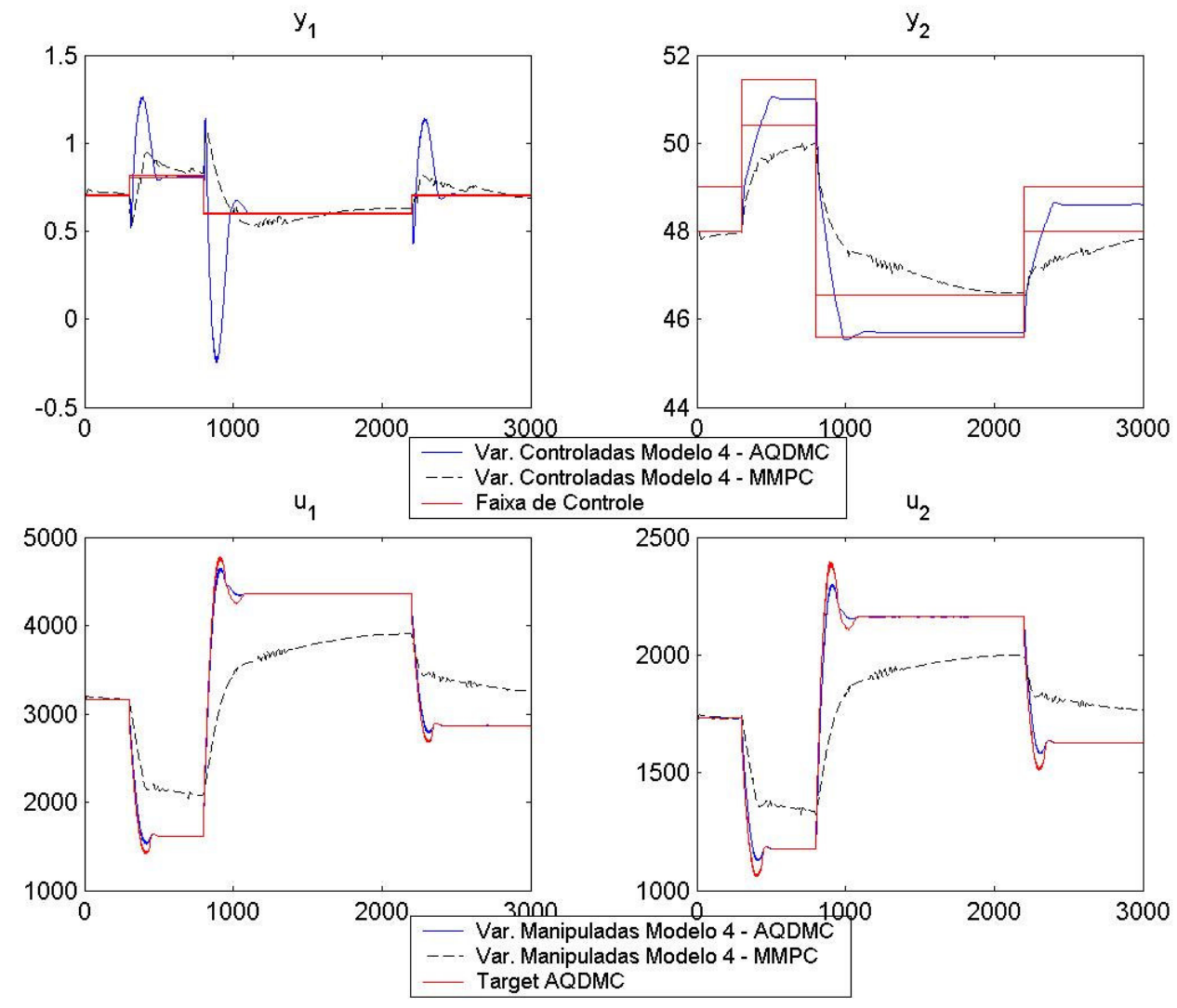

Figura 6-16 - Degrau - Planta: Modelo 4 x Controlador: Combinação entre os modelos para o pior caso

\subsubsection{Caso 6 - Planta com modelo 5}

Finalmente, na

Figura 6-17 é apresentado o resultado do teste, tanto para AQDMC quanto para MMPC, considerando que o modelo da planta é representado pelo Modelo 5. 


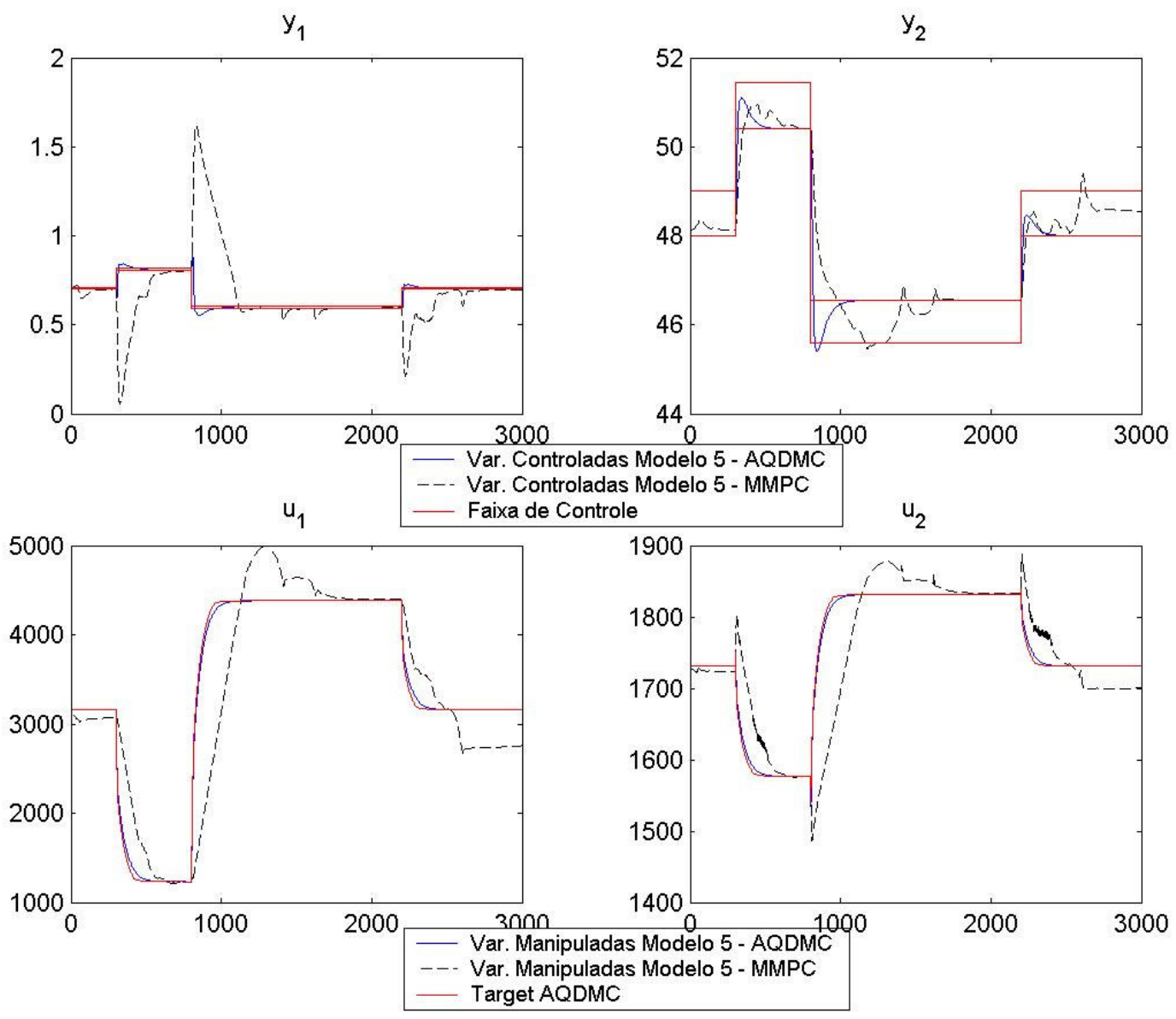

Figura 6-17 - Degrau - Planta: Modelo 5 x Controlador: Combinação entre os modelos

\subsubsection{Análise Geral}

Conforme apresentado nas figuras anteriores, o algoritmo AQDMC apresenta em todos os casos uma performance superior ao MMPC. Isto já seria esperado pois o AQDMC usa em suas predições um único modelo que está bem próximo do modelo ideal do processo. Enquanto isso, o MMPC tem as suas ações limitadas pelos piores modelos em relação ao modelo real do processo.

Para o caso 4, por exemplo, o tempo após o degrau não foi suficiente para que a resposta do controlador MMPC fosse para a nova faixa de controle, demonstrando assim, uma resposta um pouco mais lenta às perturbações.

Conforme se verifica pelo caso 5, mesmo estando o modelo do controlador adaptativo relativamente afastado da planta, a resposta do controlador AQDMC foi 
mais rápida quando comparado com o MMPC. Entretanto, o controlador AQDMC deve ser re-sintonizado para obter melhor resposta.

É possível verificar também que o comportamento das variáveis manipuladas é muito próximo entre os diferentes controladores, exceção feita em função da dinâmica de resposta. A resposta é mais rápida para as variáveis manipuladas do controlador AQDMC e mais lentas para o controlador MMPC.

\subsubsection{Identificação de Sistemas}

Está sendo considerada a abordagem definida na seção 5.2 (Comparação de modelos) para se obter o modelo ARX e posteriormente compara-lo com o modelo da planta e o respectivo modelo do controlador AQDMC.

A identificação do modelo da planta na forma de um ARX é realizada a partir dos dados de entradas e saídas, armazenados ao longo dos testes de excitação realizados na seção 6.4 (Casos de Estudo) do presente trabalho.

A análise comparativa entre estes três modelos será dada pela resposta ao degrau e pela realização da simulação destes modelos através da aplicação das entradas armazenadas tanto no modelo identificado quanto no modelo obtido pelo controlador adaptativo. Para a comparação quantitativa, serão utilizados os coeficientes de ajuste $R_{2}^{T}$ e FIT conforme apresentado em (5.2) e (5.3).

\subsubsection{Caso 1 - Planta com Modelo 1}

Para a identificação do modelo estruturado na forma de um ARX, foram utilizados os dados armazenados ao longo da simulação apresentada na seção 6.4.1 (Caso 1 Planta com Modelo 1) quando a planta foi excitada através de um sinal GBN aplicado na camada estática. O resultado da identificação é apresentado na Tabela 6-17. 
Tabela 6-17 - Modelo identificado - Planta: Modelo 1

\begin{tabular}{|l|l|l|l|l|l|l|}
\hline $\mathrm{CV}$ & $\mathrm{MV} / \mathrm{DV}$ & $\mathrm{b}_{1}$ & $\mathrm{~b}_{0}$ & $\mathrm{a}_{1}$ & $\mathrm{a}_{2}$ & $\Theta$ \\
\hline $\mathrm{C}_{3}{ }^{-}$ & $\mathrm{V}_{\mathrm{RF}}$ & $5.21 \mathrm{e}^{-5}$ & $6.84 \mathrm{e}^{-6}$ & 0.16 & 0.014 & -1 \\
\hline & $\mathrm{V}_{\mathrm{GOP}}$ & $-3.76 \mathrm{e}^{-5}$ & $-4.93 \mathrm{e}^{-5}$ & 0.16 & 0.014 & -1 \\
\hline $\mathrm{T}_{\text {TOPO }}$ & $\mathrm{V}_{\mathrm{RF}}$ & 0.0041 & -0.0011 & 1.157 & 0.069 & -1 \\
\hline & $\mathrm{V}_{\mathrm{GOP}}$ & -0.0013 & 0.0035 & 1.157 & 0.069 & -1 \\
\hline
\end{tabular}

Após a identificação dos modelos, são aplicados os testes degrau para a análise dos três modelos (identificado, planta e combinado) conforme apresentado na Figura 6-18 e Figura 6-19.

O modelo combinado a ser simulado está representado na Tabela 6-14 no caso 1 onde $\lambda_{1}=1$ conforme obtido pelo controlador adaptativo.

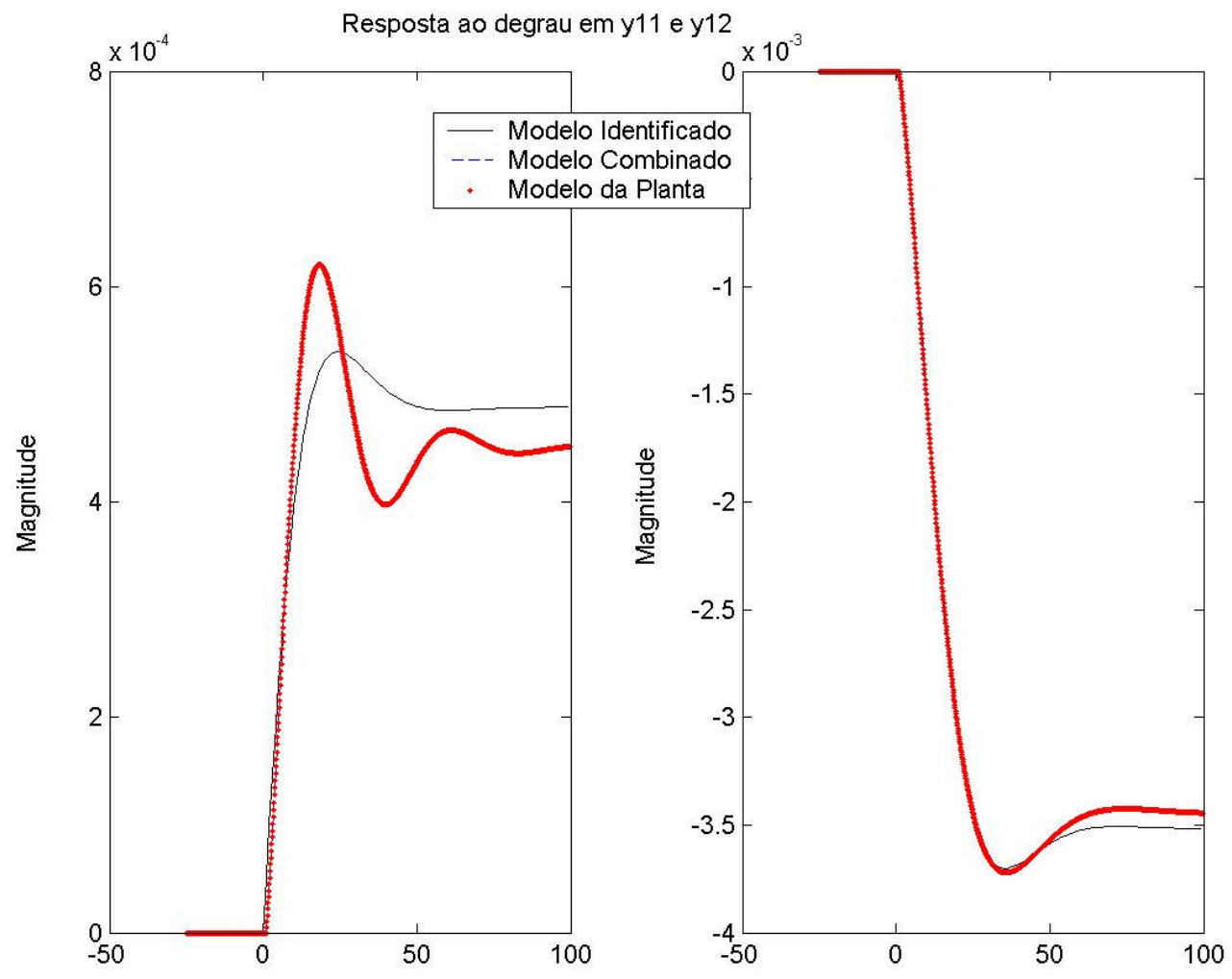

Figura 6-18 - Resposta ao Degrau para y11 e y12 respectivamente 

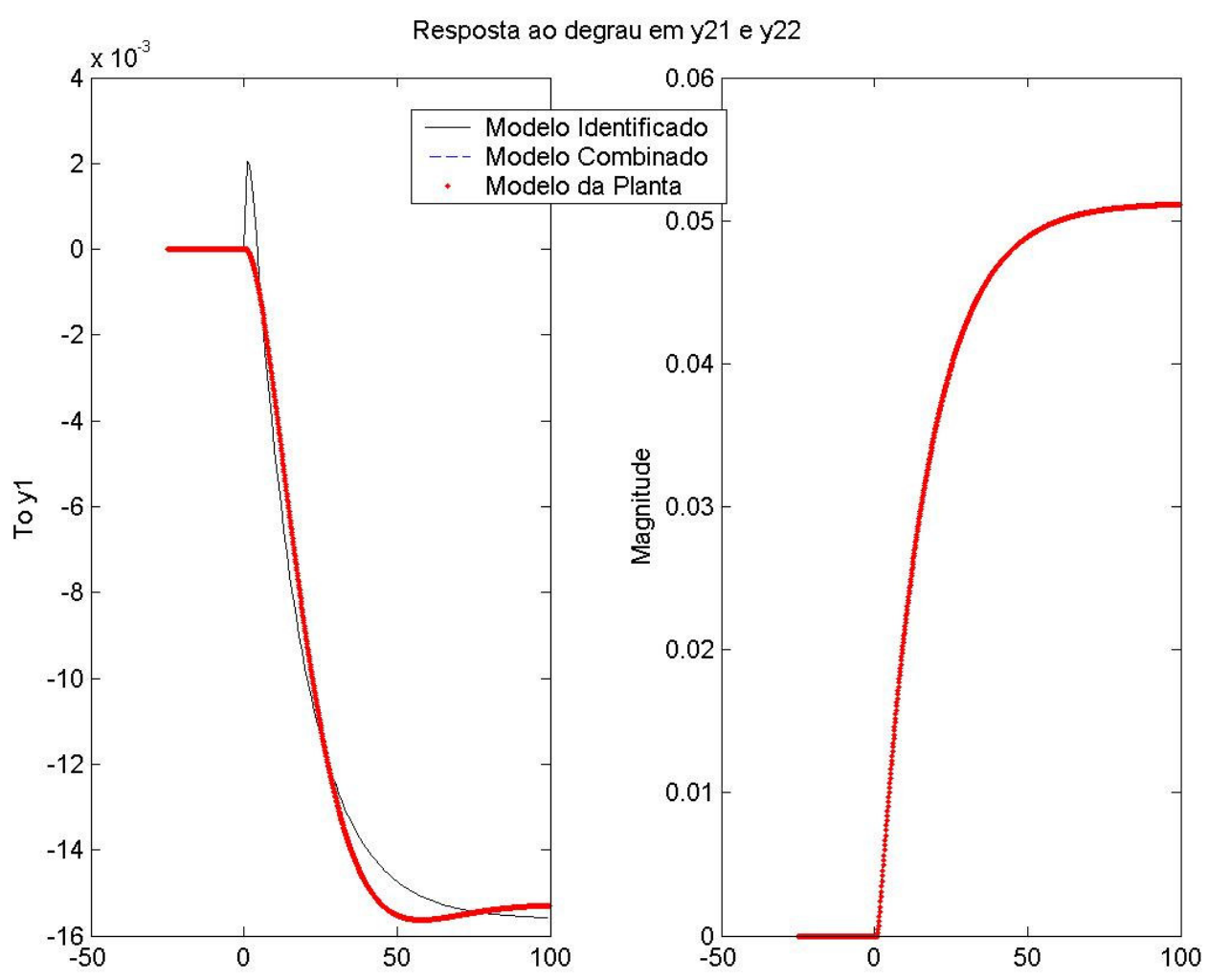

Figura 6-19 - Resposta ao Degrau para y21 e y22 respectivamente

Realizando a simulação do modelo identificado e combinado a partir das entradas armazenadas para a identificação, têm-se as Figura 6-20 e Figura 6-21. 


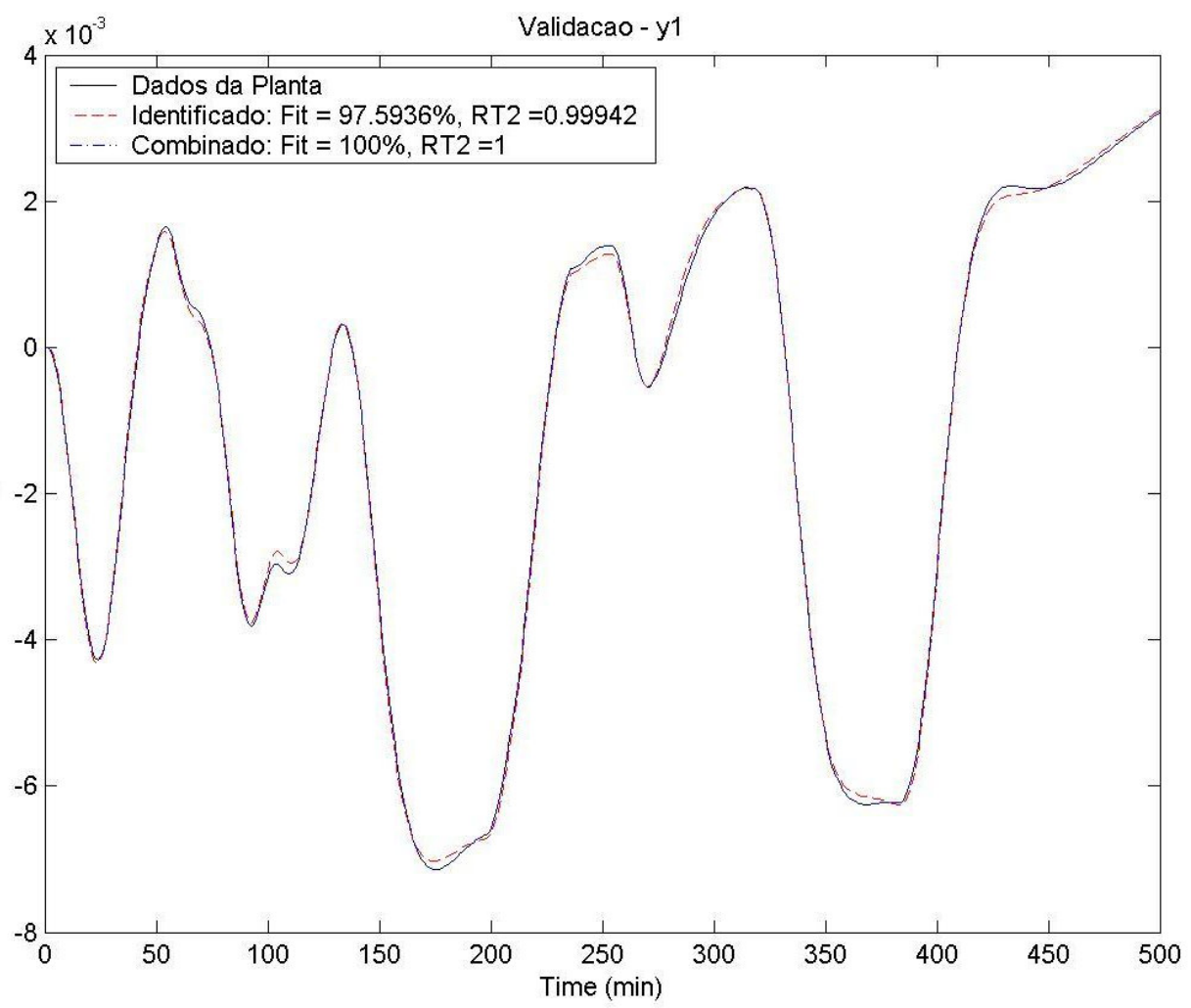

Figura 6-20 - Validação do modelo para y1

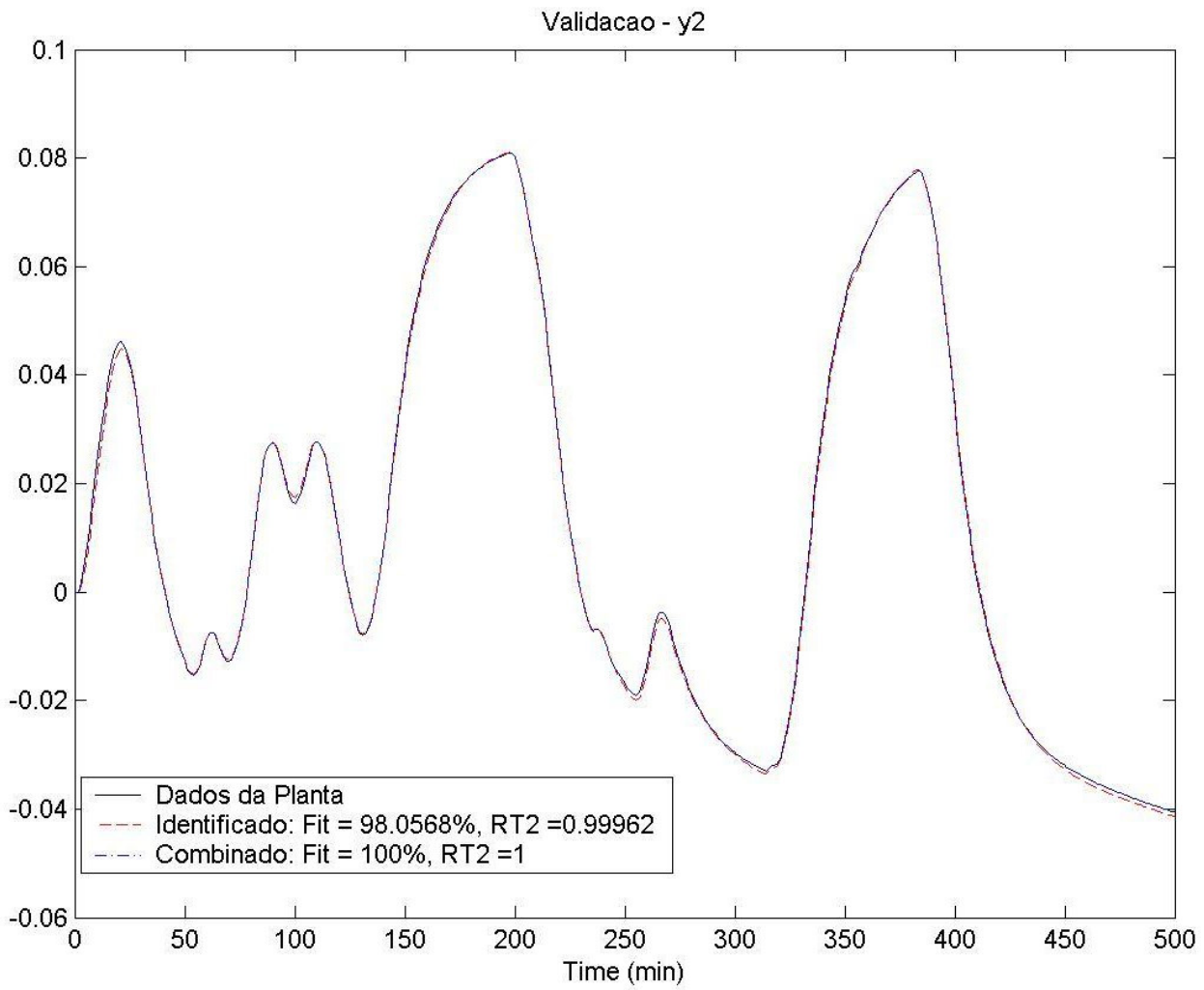

Figura 6-21 - Validação do Modelo para y2 


\subsubsection{Caso 2 - Planta com Modelo 2}

Para a identificação do modelo estruturado na forma de um ARX, foram utilizados os dados armazenados ao longo da simulação apresentada na seção 6.4.2 (Caso 2 Planta com Modelo 2) quando a planta foi excitada através de um sinal GBN aplicado na camada estática. O resultado da identificação é apresentado na Tabela 6-18.

Tabela 6-18 - Modelo identificado - Case 2

\begin{tabular}{|l|l|l|l|l|l|l|}
\hline $\mathrm{CV}$ & $\mathrm{MV} / \mathrm{DV}$ & $\mathrm{b}_{1}$ & $\mathrm{~b}_{0}$ & $\mathrm{a}_{1}$ & $\mathrm{a}_{2}$ & $\Theta$ \\
\hline $\mathrm{C}_{3}{ }^{-}$ & $\mathrm{V}_{\mathrm{RF}}$ & 0.00011 & $2.009 \mathrm{e}^{-5}$ & 0.136 & 0.0125 & -1 \\
\hline & $\mathrm{V}_{\mathrm{GOP}}$ & $-3.25 \mathrm{e}^{-5}$ & -0.00015 & 0.136 & 0.0125 & -1 \\
\hline $\mathrm{T}_{\text {TOPO }}$ & $\mathrm{V}_{\mathrm{RF}}$ & -0.0018 & -0.0016 & 0.627 & 0.059 & -1 \\
\hline & $\mathrm{V}_{\mathrm{GOP}}$ & -0.0021 & 0.0033 & 0.63 & 0.059 & -1 \\
\hline
\end{tabular}

Após a identificação dos modelos, são aplicados os testes degrau para a análise dos três modelos (identificado, planta e combinado) conforme apresentado na Figura 6-22 e Figura 6-23.

O modelo combinado a ser simulado está representado na Tabela 6-14 no caso 2 onde $\lambda_{2}=1$ conforme obtido pelo controlador adaptativo. 


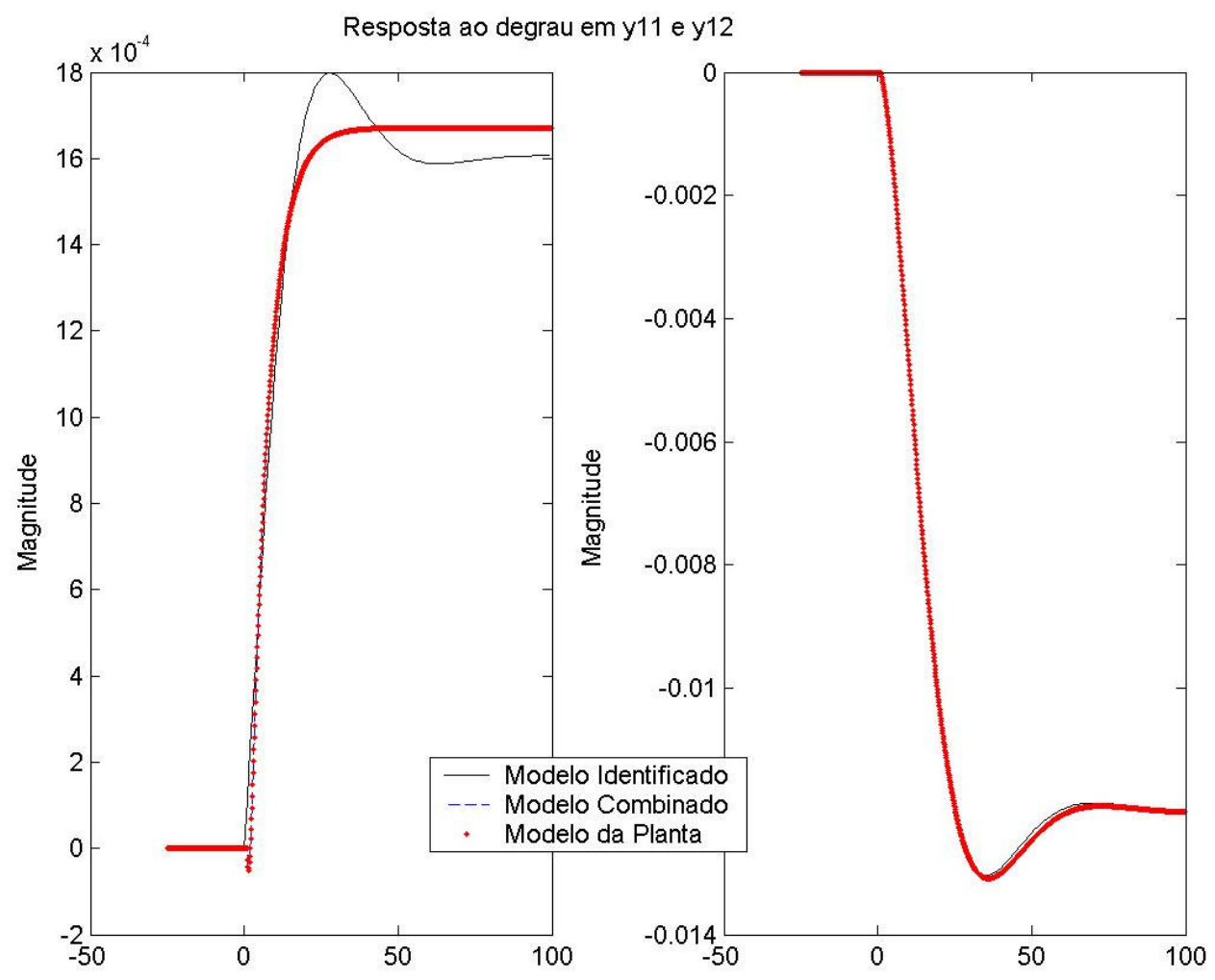

Figura 6-22 - Resposta ao Degrau para y11 e y12 respectivamente

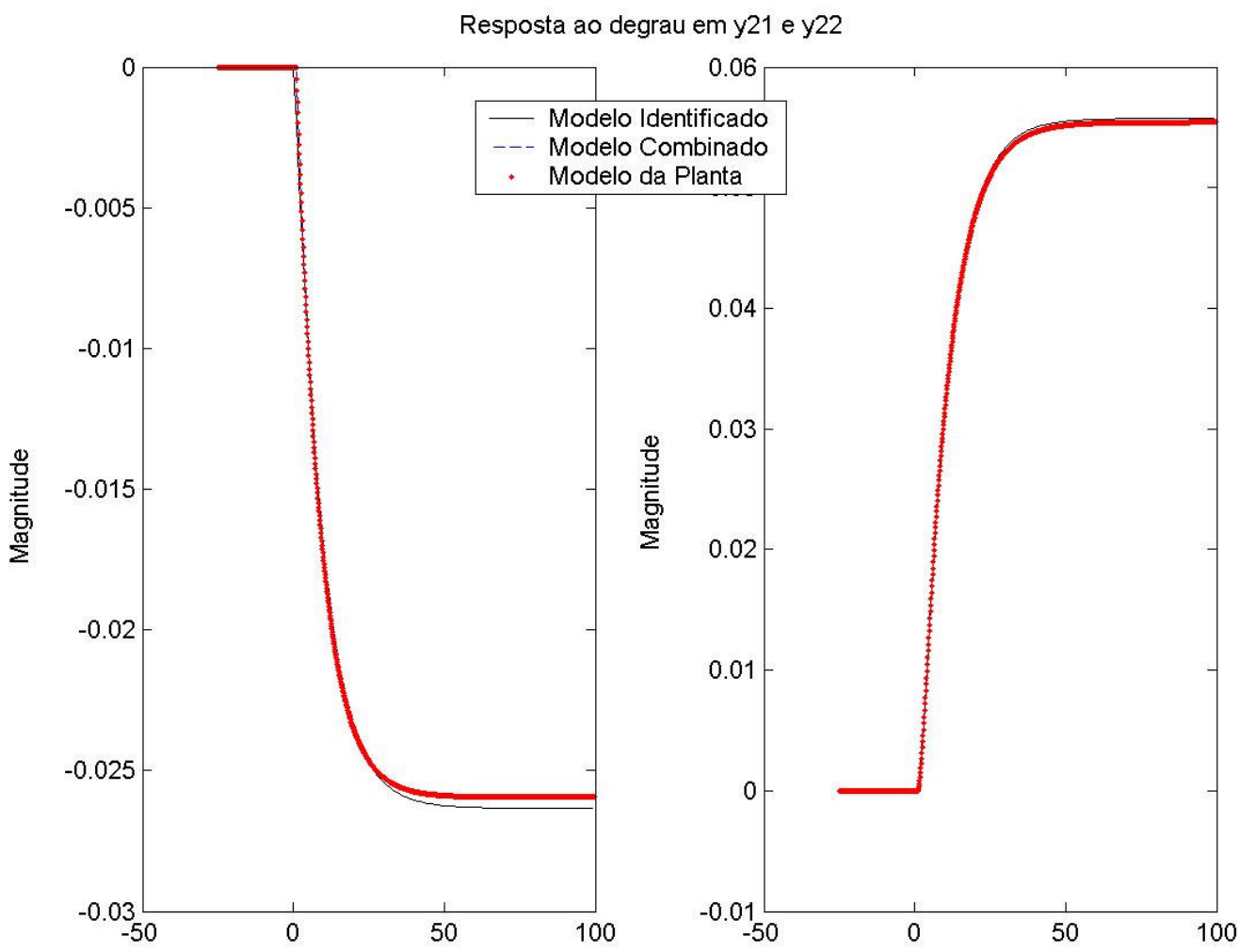

Figura 6-23 - Resposta ao Degrau para y21 e y22 respectivamente 
Realizando a simulação do modelo identificado e combinado a partir das entradas armazenadas para a identificação, têm-se Figura 6-24 e Figura 6-25.

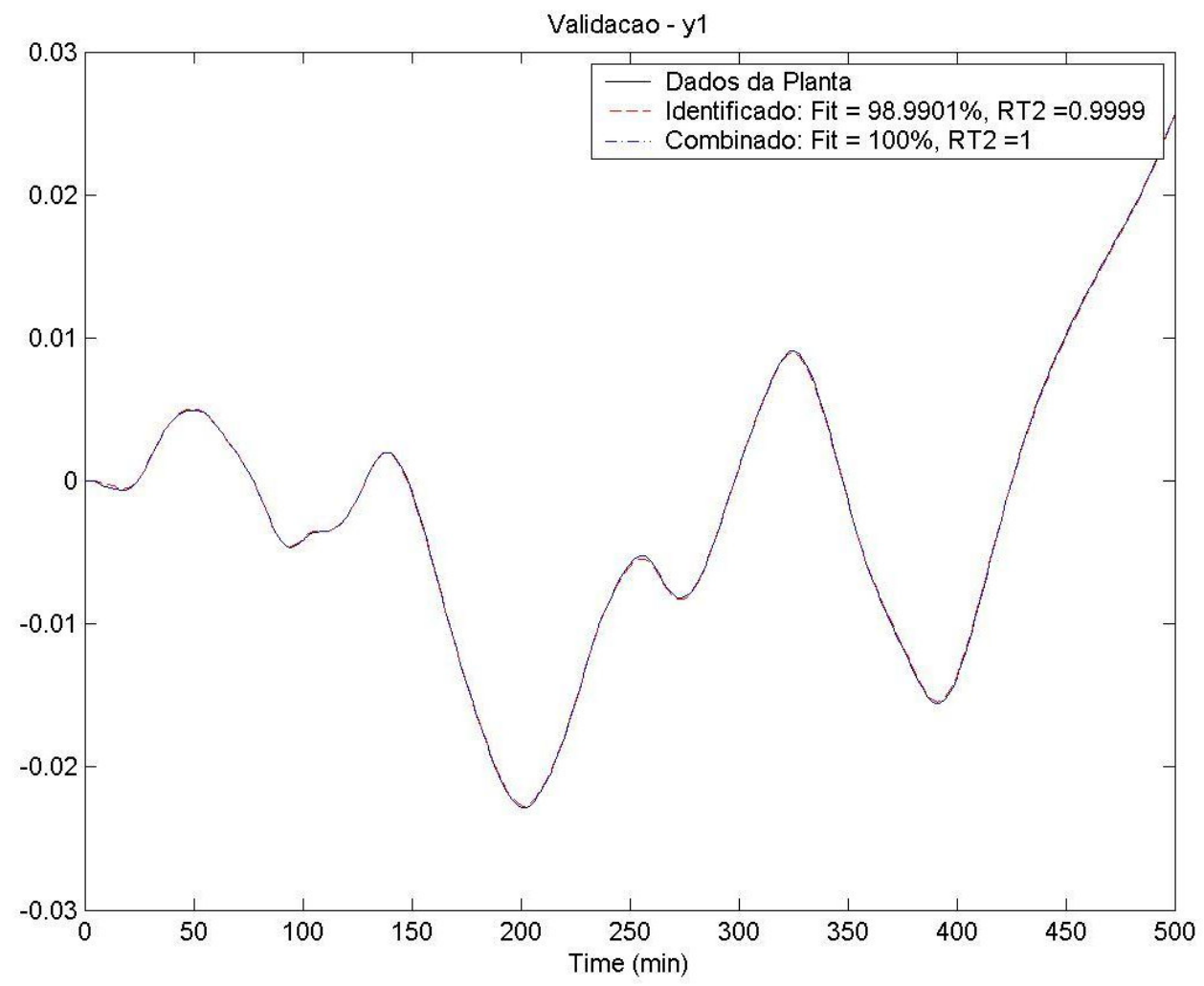

Figura 6-24 - Validação do modelo para y1 


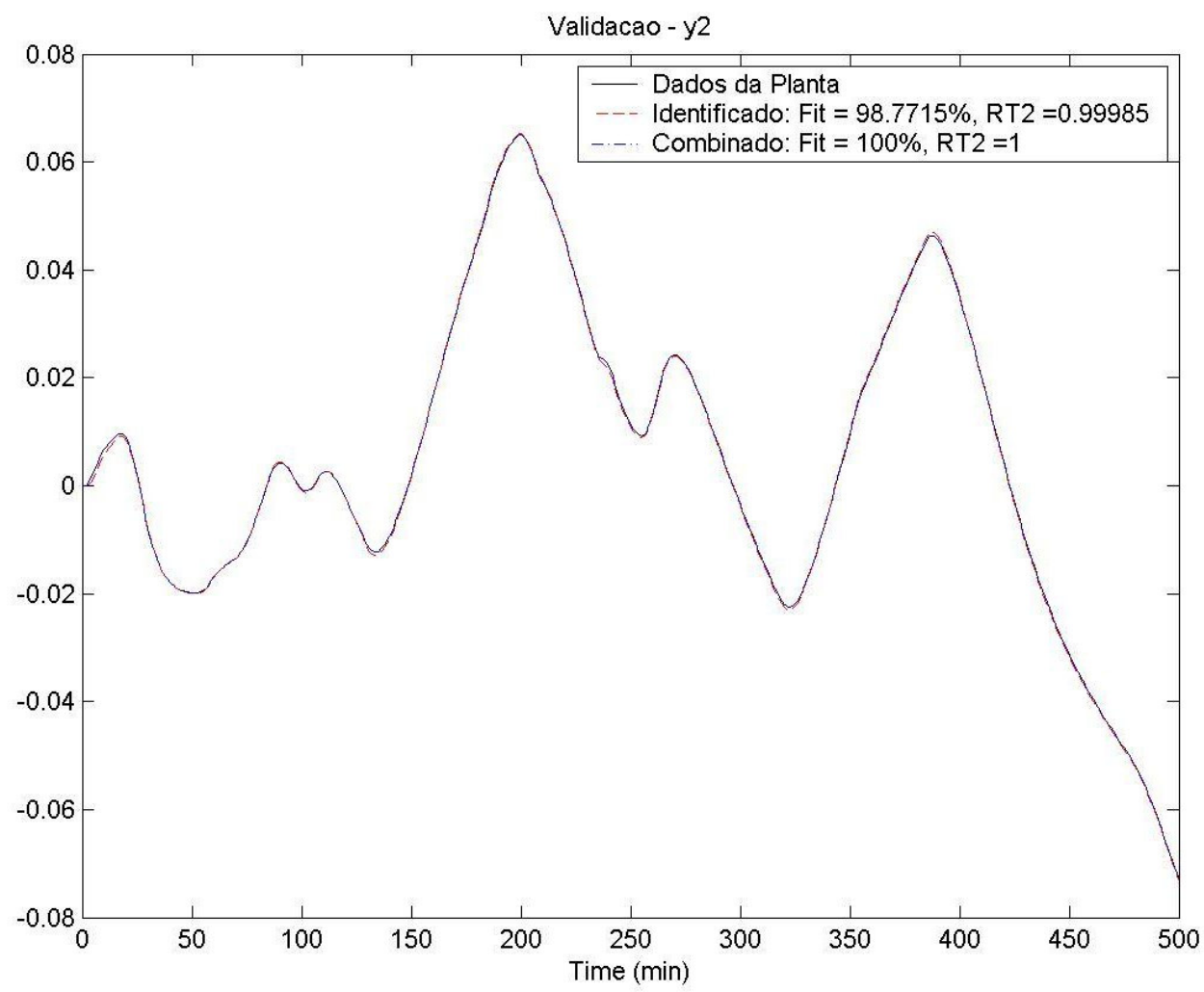

Figura 6-25 - Validação do Modelo para y2

\subsubsection{Caso 3 - Planta com Modelo 3}

Para a identificação do modelo estruturado na forma de um ARX, foram utilizados os dados armazenados ao longo da simulação apresentada na seção 6.4 .3 (Caso 3 Planta com Modelo 3) quando a planta foi excitada através de um sinal GBN aplicado na camada estática. O resultado da identificação é apresentado na Tabela 6-19.

Tabela 6-19 - Modelo identificado - Case 3

\begin{tabular}{|l|l|l|l|l|l|l|}
\hline $\mathrm{CV}$ & $\mathrm{MV} / \mathrm{DV}$ & $\mathrm{b}_{1}$ & $\mathrm{~b}_{0}$ & $\mathrm{a}_{1}$ & $\mathrm{a}_{2}$ & $\Theta$ \\
\hline $\mathrm{C}_{3}{ }^{-}$ & $\mathrm{V}_{\mathrm{RF}}$ & 0.00061 & 0.00032 & 0.3396 & 0.017 & -1 \\
\hline & $\mathrm{V}_{\mathrm{GOP}}$ & 0.00040 & -0.00078 & 0.3396 & 0.017 & -1 \\
\hline $\mathrm{T}_{\mathrm{TOPO}}$ & $\mathrm{V}_{\mathrm{RF}}$ & -0.0044 & -0.0039 & 1.69 & 0.17 & -1 \\
\hline & $\mathrm{V}_{\mathrm{GOP}}$ & -0.0027 & 0.0080 & 1.69 & 0.17 & -1 \\
\hline
\end{tabular}

Após a identificação dos modelos, são aplicados os testes degrau para a análise dos três modelos (identificado, planta e combinado) conforme apresentado na Figura 6-26 e Figura 6-27. 
O modelo combinado a ser simulado está representado na Tabela 6-14 no caso 3 onde $\lambda_{3}=0.93$ conforme obtido pelo controlador adaptativo.

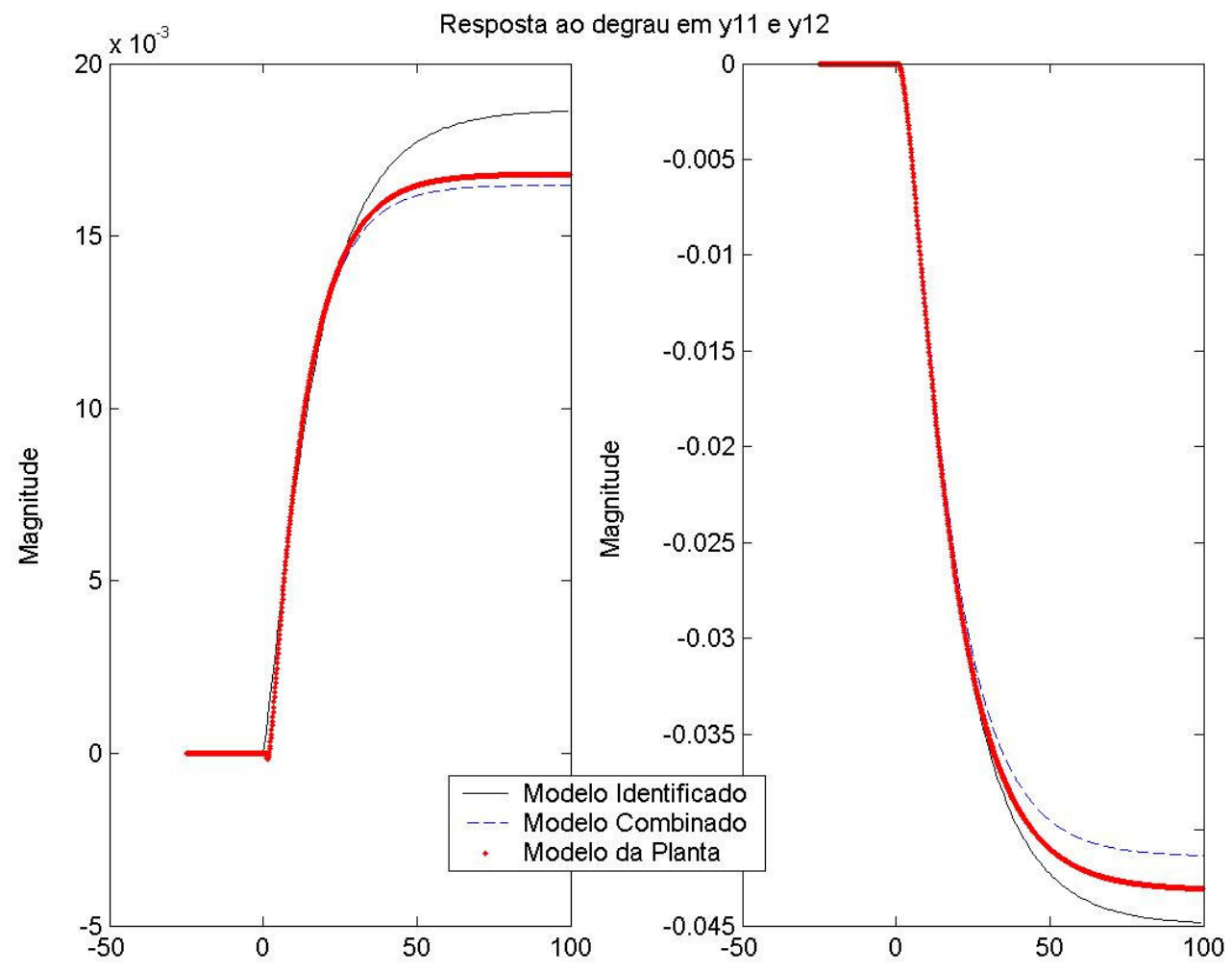

Figura 6-26 - Resposta ao Degrau para y11 e y12 respectivamente 


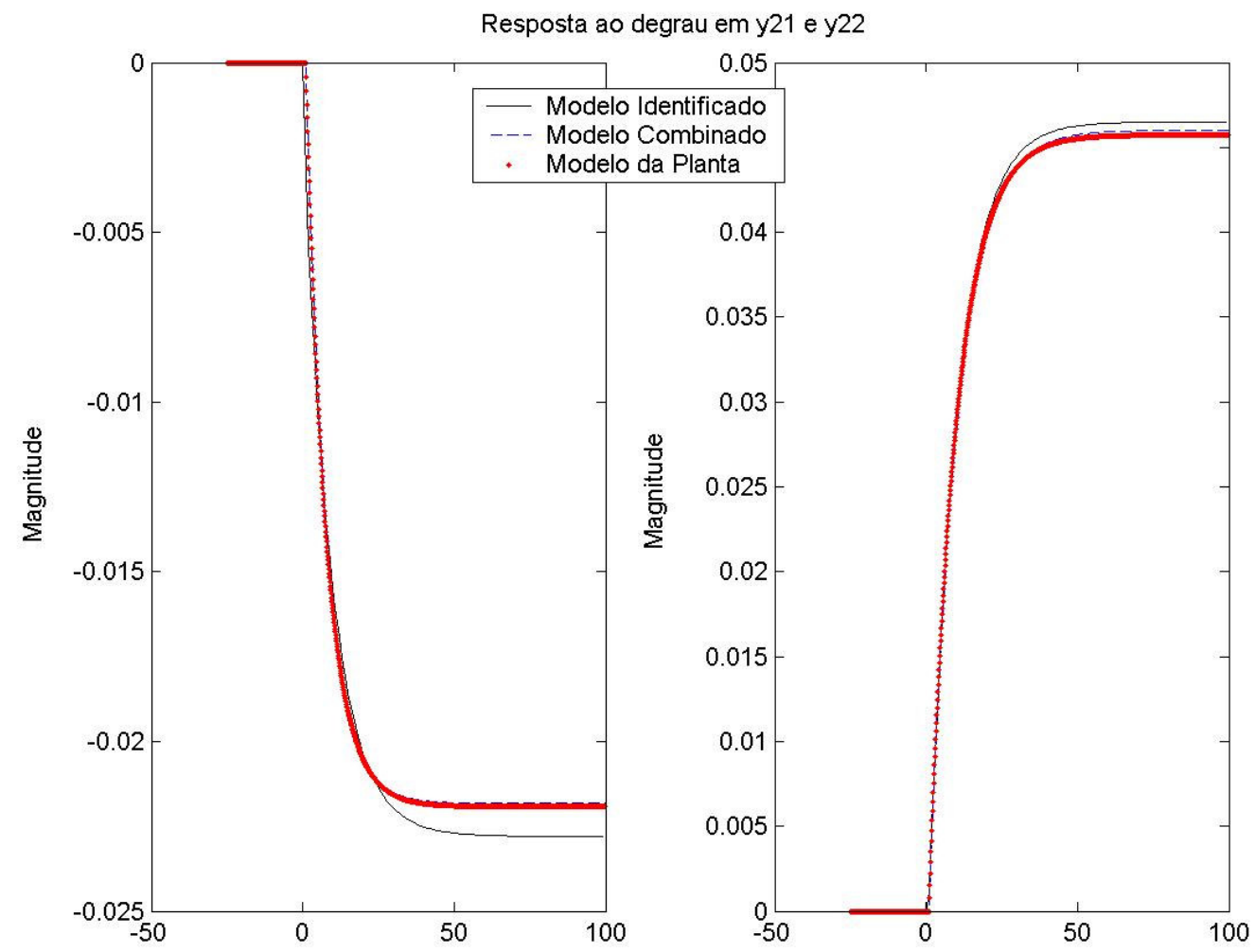

Figura 6-27 - Resposta ao Degrau para y21 e y22 respectivamente

Realizando a simulação do modelo identificado e combinado a partir das entradas armazenadas para a identificação, têm-se Figura 6-28 e

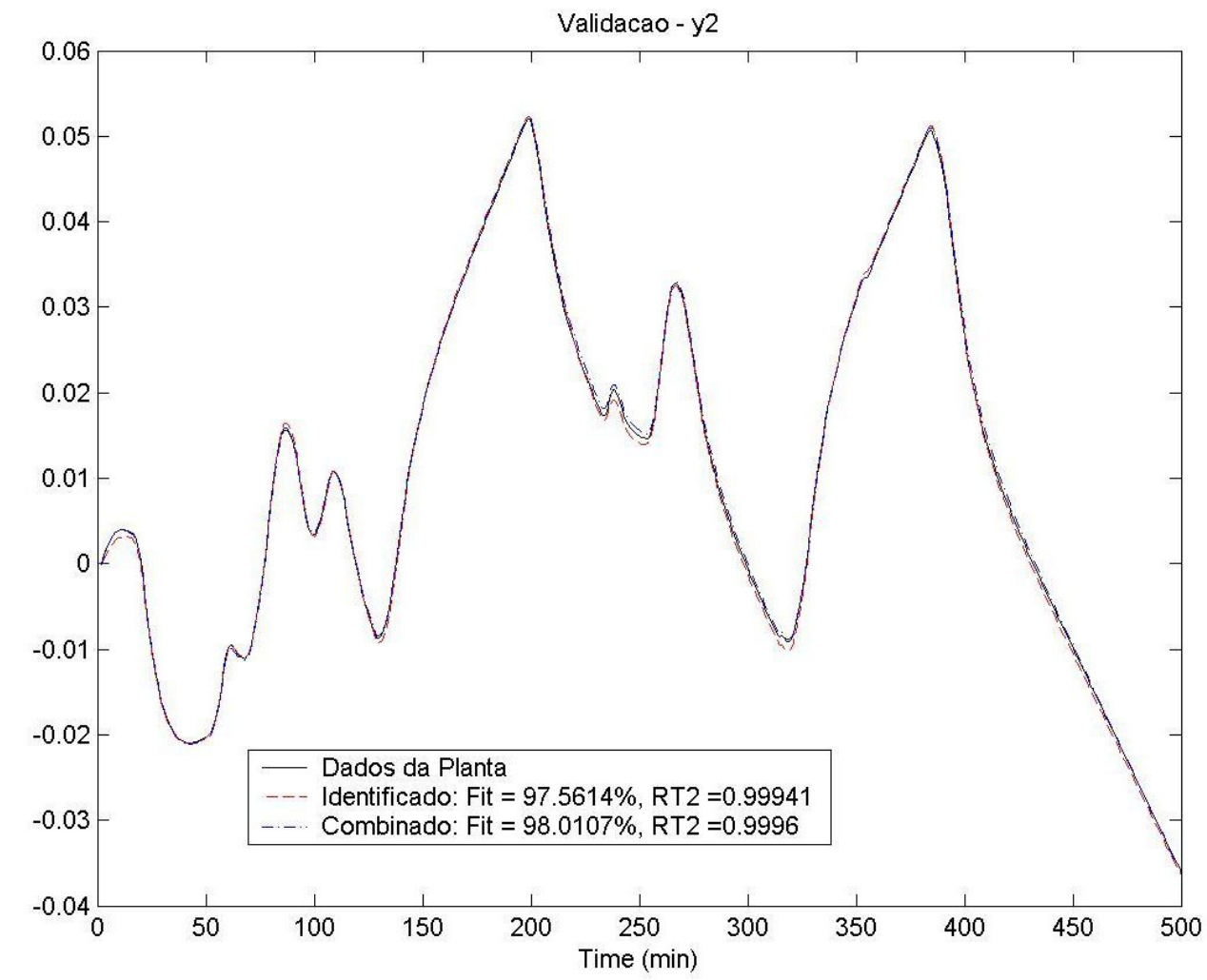


Figura 6-29.

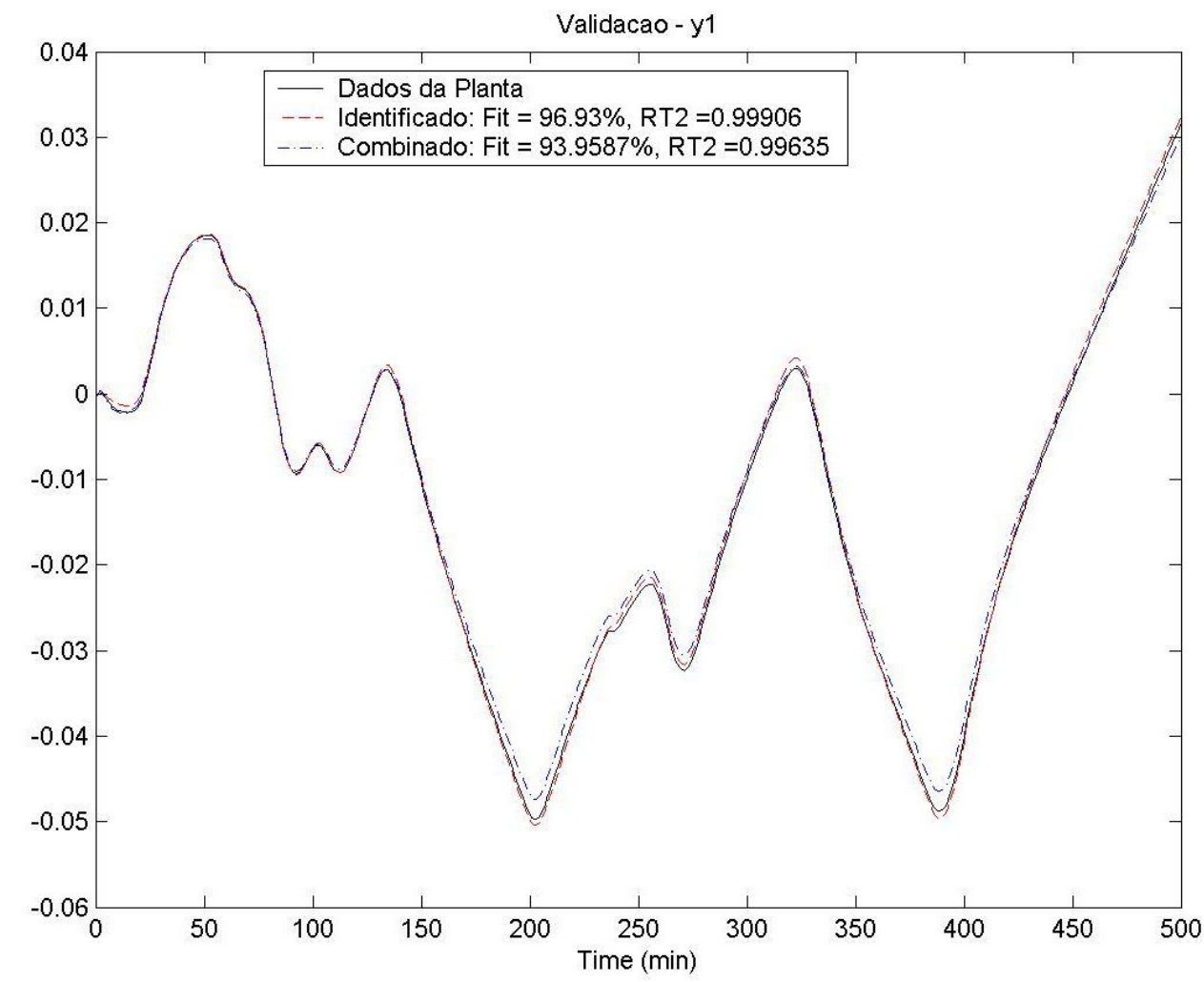

Figura 6-28 - Validação do modelo para y1

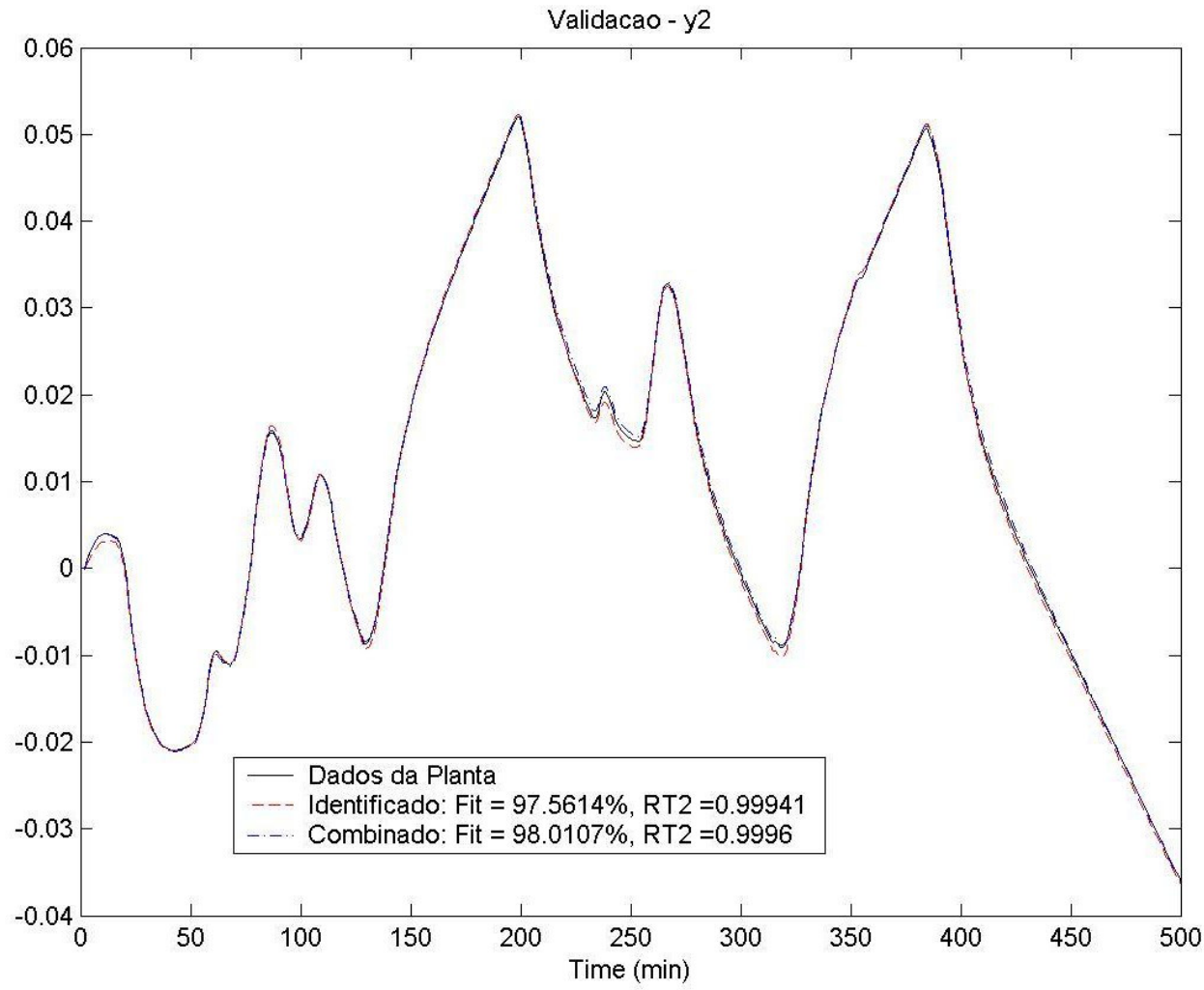


Figura 6-29 - Validação do Modelo para y2

\subsubsection{Caso 4 - Planta com Modelo 4}

Para a identificação do modelo estruturado na forma de um ARX, foram utilizados os dados armazenados ao longo da simulação apresentada na seção 6.4.4 (Caso 4 Planta com Modelo 4) quando a planta foi excitada através de um sinal GBN aplicado na camada estática. O resultado da identificação é apresentado na Tabela 6-20.

Tabela 6-20 - Modelo identificado - Case 4

\begin{tabular}{|l|l|l|l|l|l|l|}
\hline $\mathrm{CV}$ & $\mathrm{MV} / \mathrm{DV}$ & $\mathrm{b}_{1}$ & $\mathrm{~b}_{0}$ & $\mathrm{a}_{1}$ & $\mathrm{a}_{2}$ & $\Theta$ \\
\hline $\mathrm{C}_{3}{ }^{-}$ & $\mathrm{V}_{\mathrm{RF}}$ & 0.0004 & $6.99 \mathrm{e}^{-5}$ & 0.23 & 0.0075 & -1 \\
\hline & $\mathrm{V}_{\mathrm{GOP}}$ & $-2.23 \mathrm{e}^{-5}$ & -0.00017 & 0.23 & 0.0075 & -1 \\
\hline $\mathrm{T}_{\mathrm{TOPO}}$ & $\mathrm{V}_{\mathrm{RF}}$ & -0.0032 & -0.00025 & 0.1995 & 0.0077 & -1 \\
\hline & $\mathrm{V}_{\mathrm{GOP}}$ & 0.0030 & 0.00059 & 0.2 & 0.0077 & -1 \\
\hline
\end{tabular}

Após a identificação dos modelos, são aplicados os testes degrau para a análise dos três modelos (identificado, planta e combinado) conforme apresentado na Figura 6-30 e Figura 6-31.

O modelo combinado a ser simulado está representado na Tabela 6-14 no caso 4 onde $\lambda_{4}=0.99$ conforme obtido pelo controlador adaptativo. 

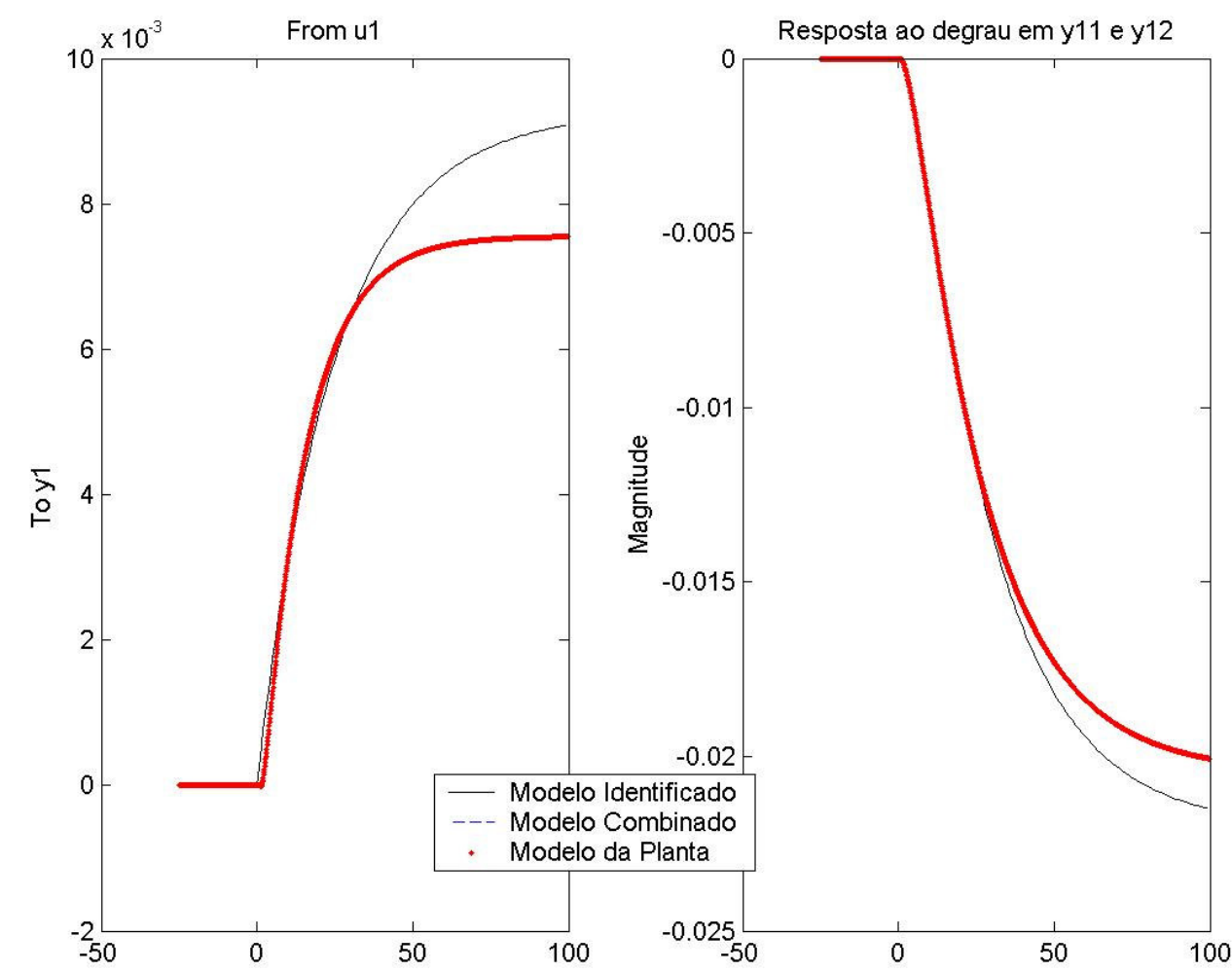

Figura 6-30 - Resposta ao Degrau para y11 e y12 respectivamente

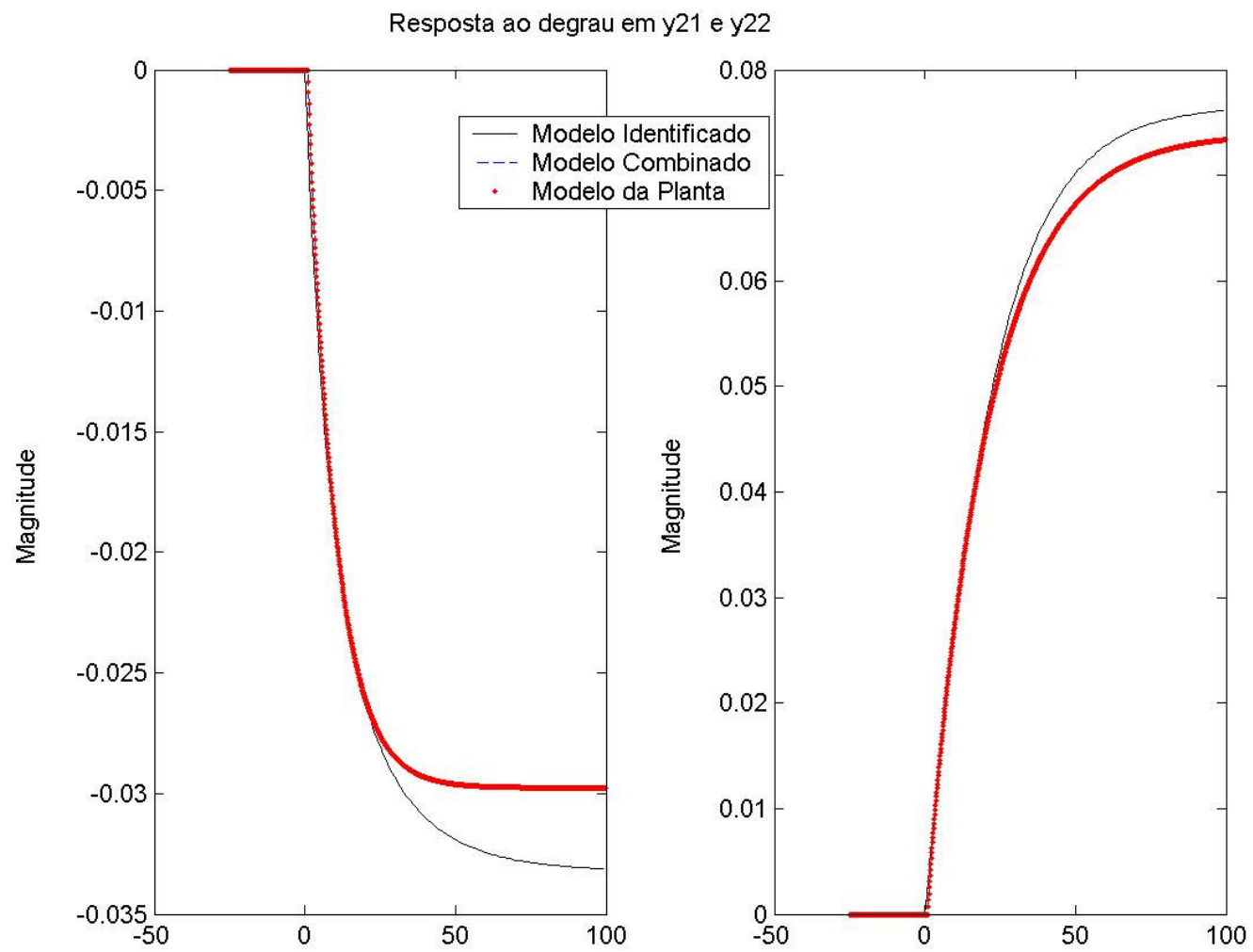

Figura 6-31 - Resposta ao Degrau para y21 e y22 respectivamente 
Realizando a simulação do modelo identificado e combinado a partir das entradas armazenadas para a identificação, têm-se Figura 6-32 e Figura 6-33.

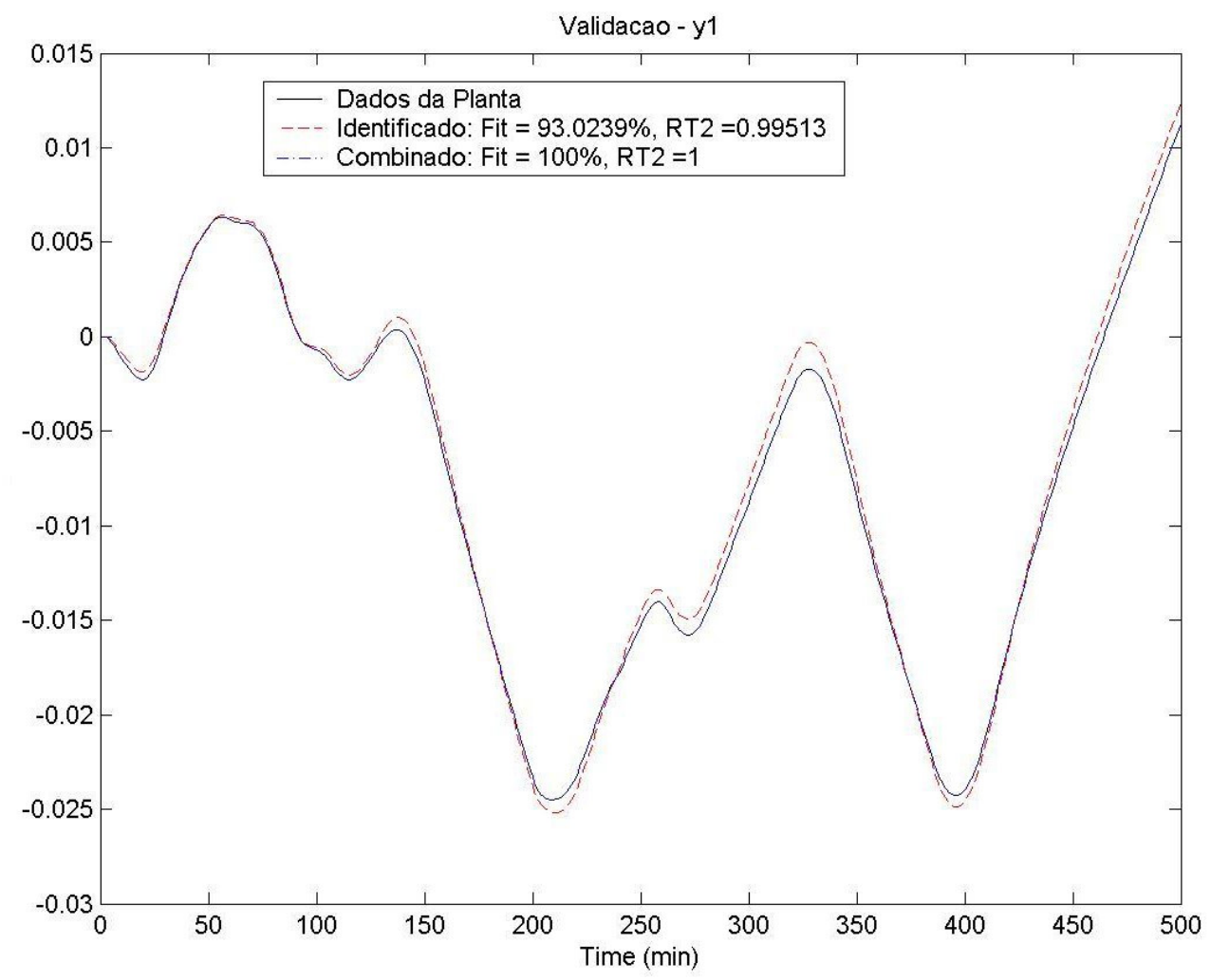

Figura 6-32 - Validação do modelo para y1 


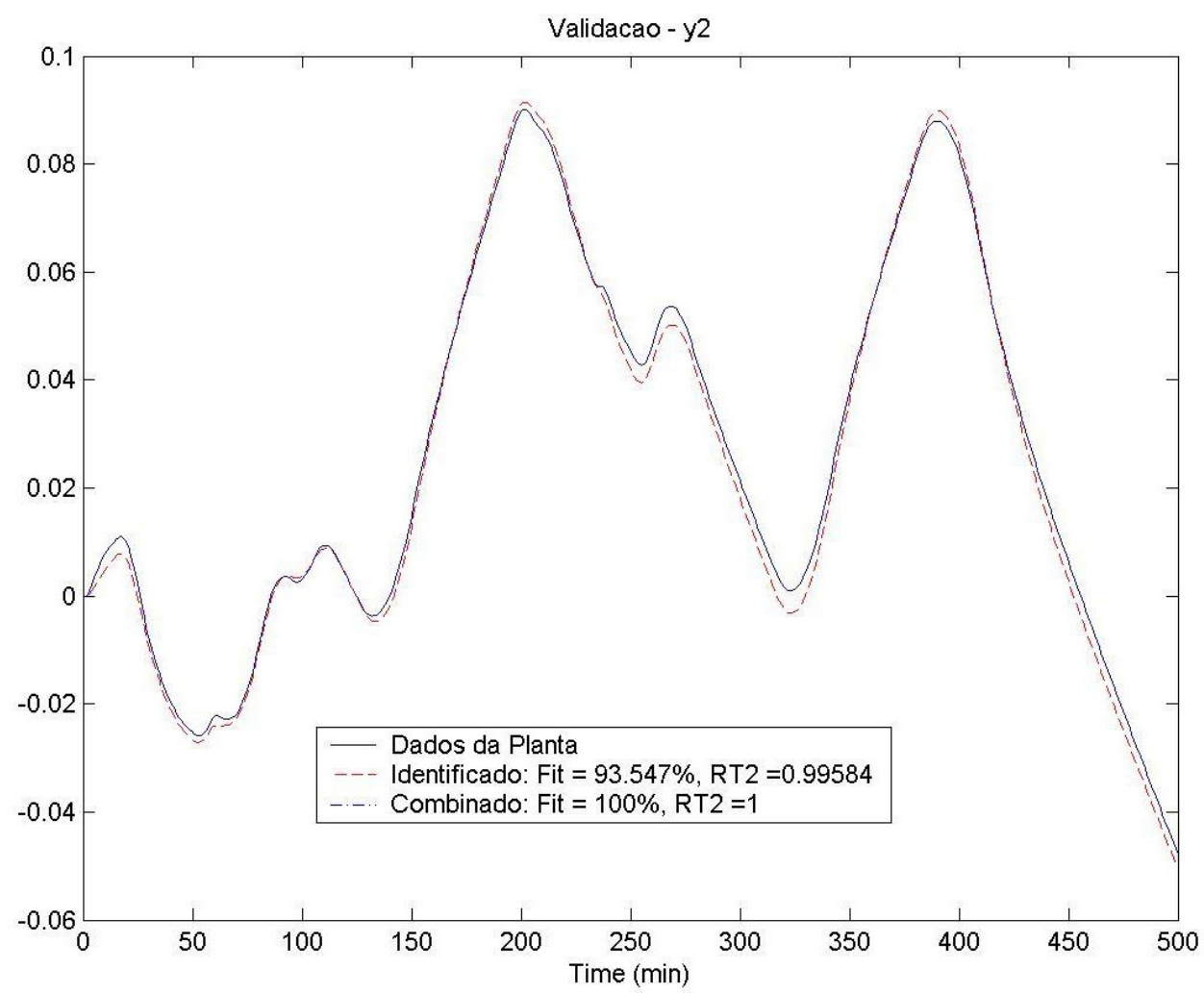

Figura 6-33 - Validação do Modelo para y2

\subsubsection{Caso 5 - Planta com Modelo 4}

Neste teste o modelo do controlador a ser considerado possui como característica o fato de estar distante do modelo da planta como pode ser verificado na Tabela 6-14.

Para a identificação do modelo estruturado na forma de um ARX, foram utilizados os dados armazenados ao longo da simulação apresentada na seção 6.4.4 (Caso 4 Planta com Modelo 4) quando a planta foi excitada através de um sinal GBN aplicado na camada estática. O resultado da identificação é apresentado na Tabela 6-21.

Tabela 6-21 - Modelo identificado - Case 5

\begin{tabular}{|l|l|l|l|l|l|l|}
\hline $\mathrm{CV}$ & $\mathrm{MV} / \mathrm{DV}$ & $\mathrm{b}_{1}$ & $\mathrm{~b}_{0}$ & $\mathrm{a}_{1}$ & $\mathrm{a}_{2}$ & $\Theta$ \\
\hline $\mathrm{C}_{3}{ }^{-}$ & $\mathrm{V}_{\mathrm{RF}}$ & 0.00039 & $7.15 \mathrm{e}^{-5}$ & 0.236 & 0.0077 & -1 \\
\hline & $\mathrm{V}_{\mathrm{GOP}}$ & $-1.47 \mathrm{e}^{-5}$ & -0.00017 & 0.24 & 0.0077 & -1 \\
\hline $\mathrm{T}_{\text {TOPO }}$ & $\mathrm{V}_{\mathrm{RF}}$ & -0.0032 & -0.00027 & 0.21 & 0.008 & -1 \\
\hline & $\mathrm{V}_{\mathrm{GOP}}$ & 0.003 & 0.00062 & 0.21 & 0.008 & -1 \\
\hline
\end{tabular}


Após a identificação dos modelos, são aplicados os testes degrau para a análise dos três modelos (identificado, planta e combinado) conforme apresentado na Figura 6-34 e Figura 6-35.

O modelo combinado a ser simulado está representado na Tabela 6-14 no caso 5 onde $\lambda_{4}=0.63$ conforme obtido pelo controlador adaptativo.

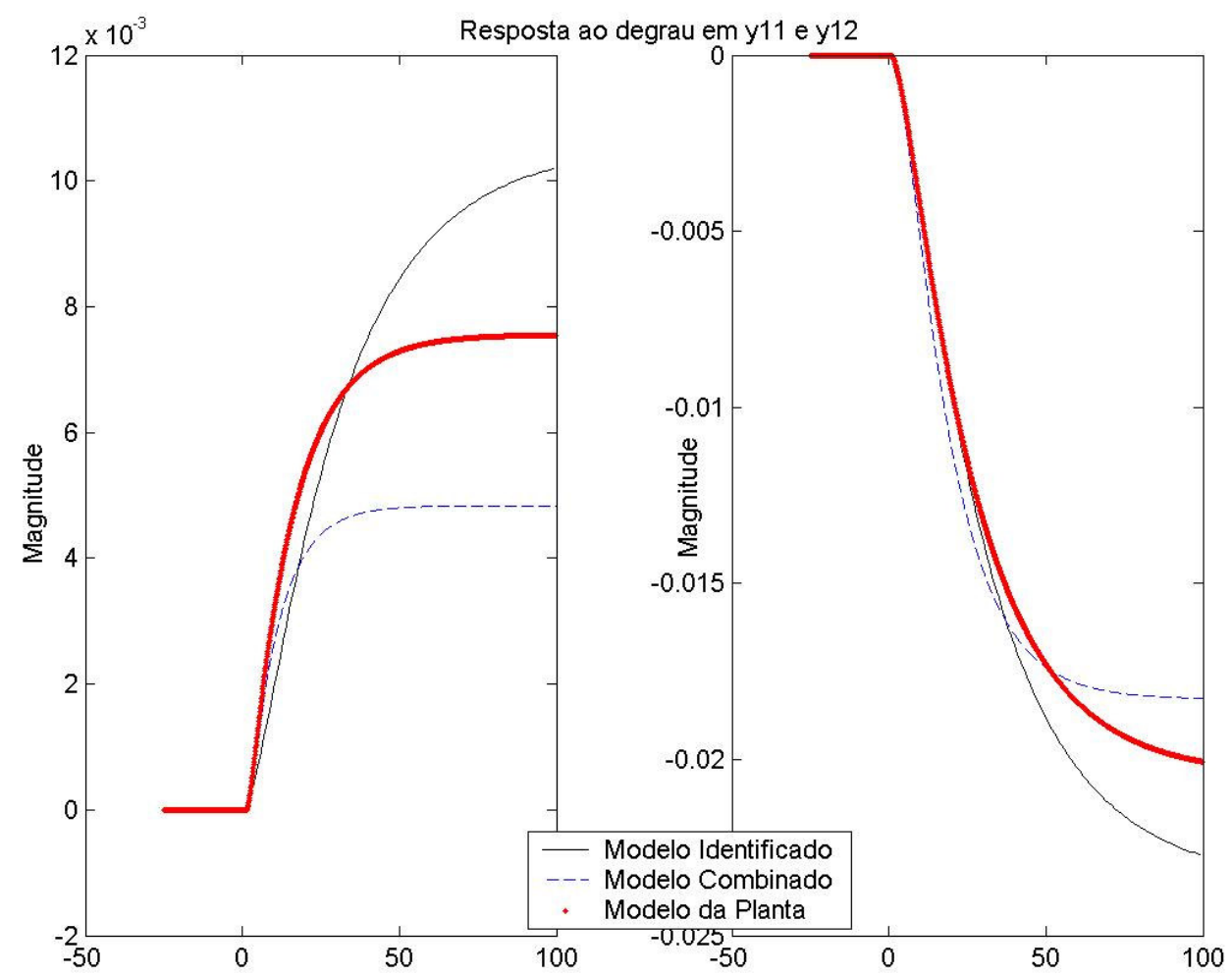

Figura 6-34 - Resposta ao Degrau para y11 e y12 respectivamente 


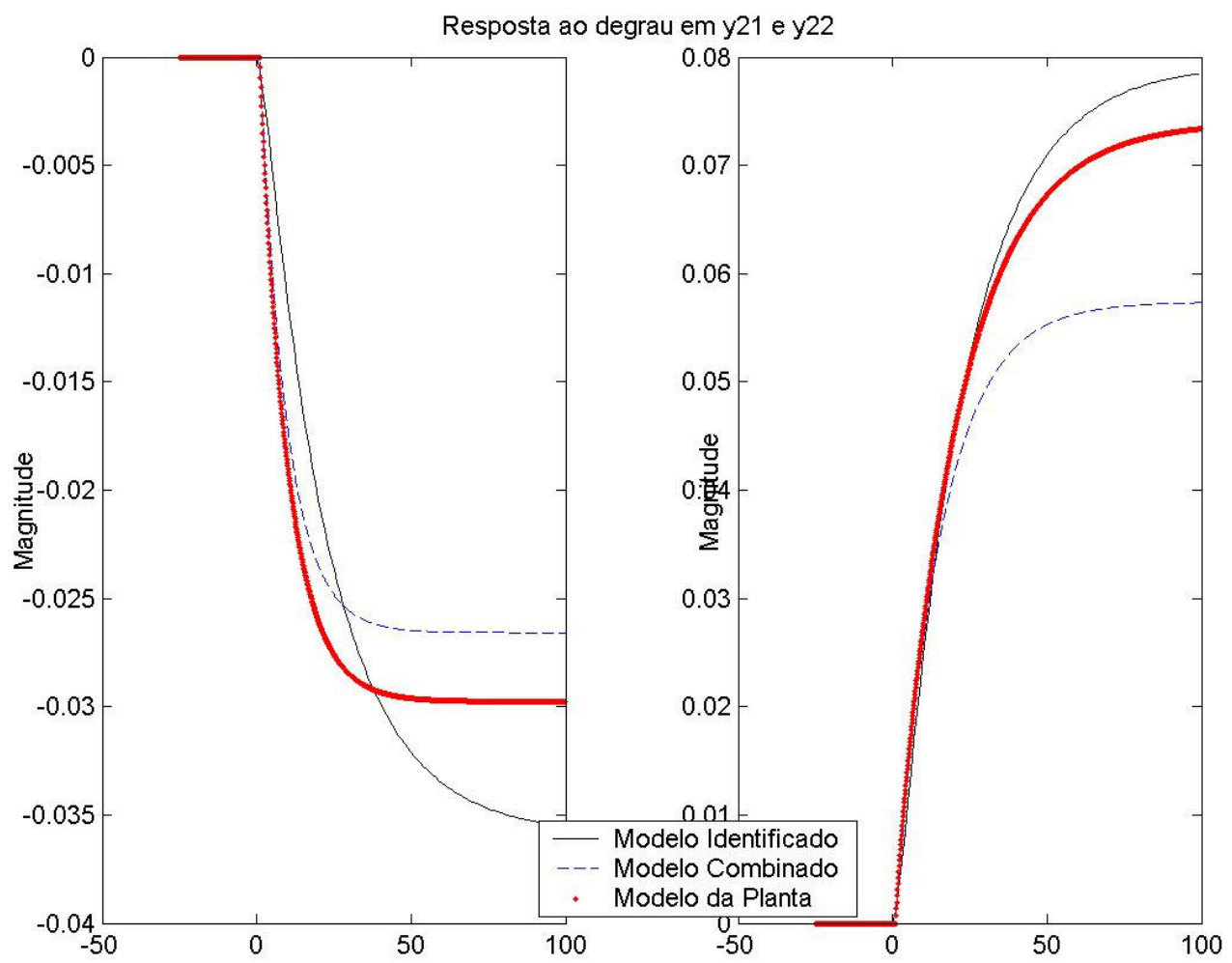

Figura 6-35 - Resposta ao Degrau para y21 e y22 respectivamente

Realizando a simulação do modelo identificado e combinado a partir das entradas armazenadas para a identificação, têm-se Figura 6-36 e Figura 6-37. 


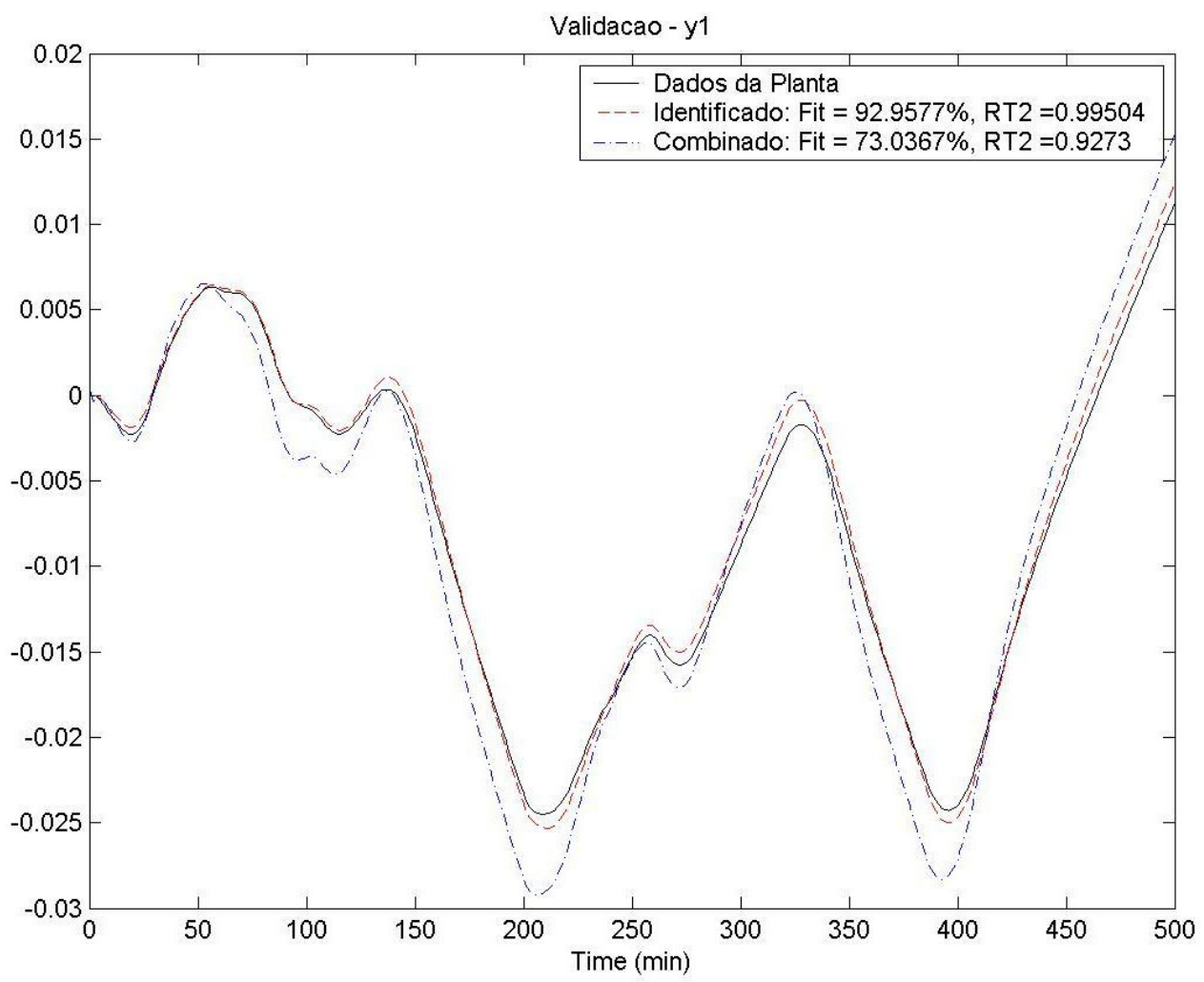

Figura 6-36 - Validação do modelo para y1

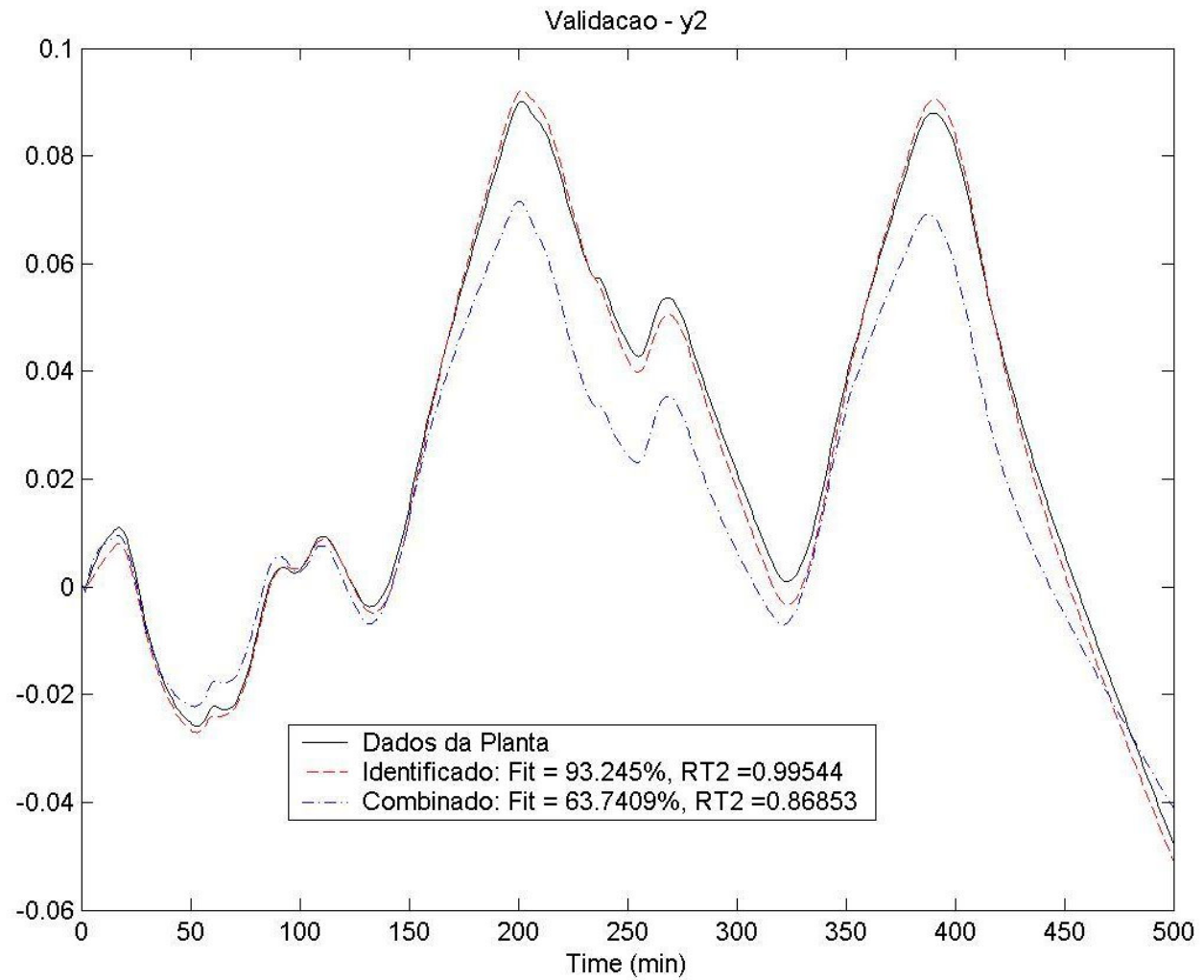

Figura 6-37 - Validação do Modelo para y2 


\subsubsection{Caso 6 - Planta com Modelo 5}

Para a identificação do modelo estruturado na forma de um ARX, foram utilizados os dados armazenados ao longo da simulação apresentada na seção 6.4 .5 (Caso 5 Planta com Modelo 5) quando a planta foi excitada através de um sinal GBN aplicado na camada estática. O resultado da identificação é apresentado na Tabela 6-22.

Tabela 6-22 - Modelo identificado - Case 6

\begin{tabular}{|l|l|l|l|l|l|l|}
\hline $\mathrm{CV}$ & $\mathrm{MV} / \mathrm{DV}$ & $\mathrm{b}_{1}$ & $\mathrm{~b}_{0}$ & $\mathrm{a}_{1}$ & $\mathrm{a}_{2}$ & $\Theta$ \\
\hline $\mathrm{C}_{3}{ }^{-}$ & $\mathrm{V}_{\mathrm{RF}}$ & 0.0002 & 0.00018 & 0.34 & 0.026 & -1 \\
\hline & $\mathrm{V}_{\mathrm{GOP}}$ & 0.00032 & -0.00047 & 0.34 & 0.026 & -1 \\
\hline $\mathrm{T}_{\text {TOPO }}$ & $\mathrm{V}_{\mathrm{RF}}$ & -0.0047 & -0.0034 & 2.29 & 0.17 & -1 \\
\hline & $\mathrm{V}_{\mathrm{GOP}}$ & -0.0035 & 0.0078 & 2.29 & 0.17 & -1 \\
\hline
\end{tabular}

Após a identificação dos modelos, são aplicados os testes degrau para a análise dos três modelos (identificado, planta e combinado) conforme apresentado na Figura 6-38 e Figura 6-39.

O modelo combinado a ser simulado está representado na Tabela 6-14 no caso 6 onde $\lambda_{5}=0.99$ conforme obtido pelo controlador adaptativo. 


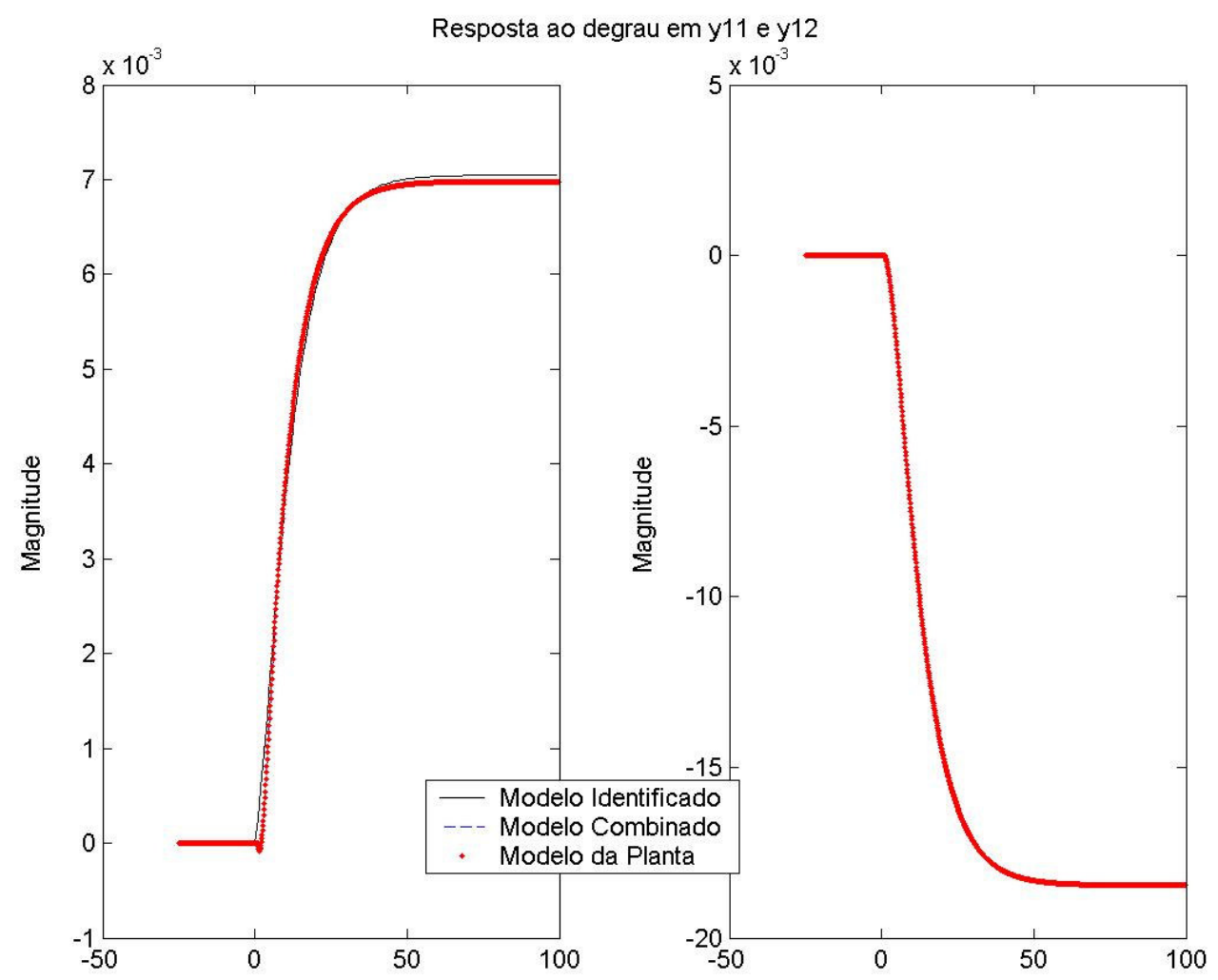

Figura 6-38 - Resposta ao Degrau para y11 e y12 respectivamente

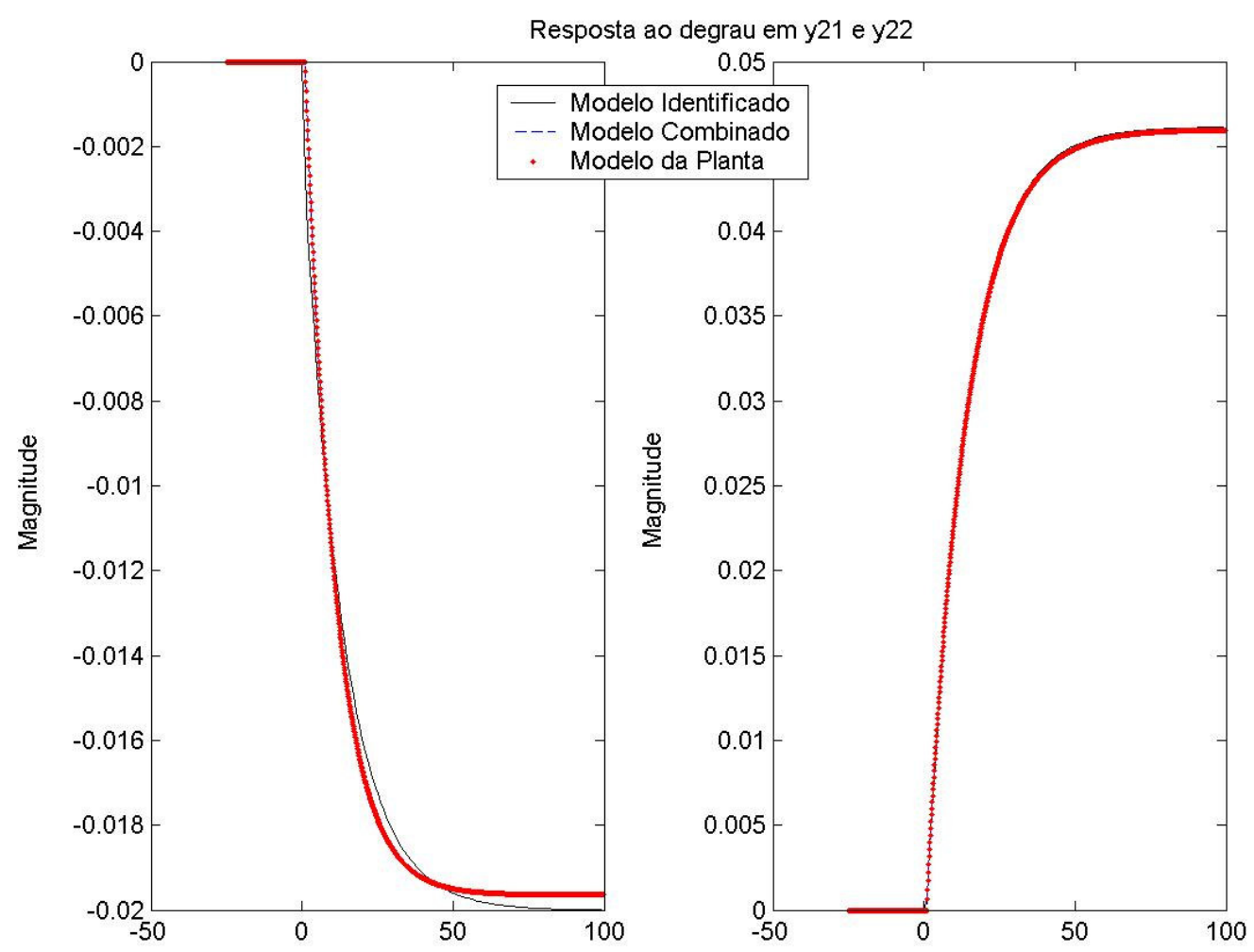

Figura 6-39 - Resposta ao Degrau para y21 e y22 respectivamente 
Realizando a simulação do modelo identificado e combinado a partir das entradas armazenadas para a identificação, têm-se Figura 6-40 e Figura 6-41.

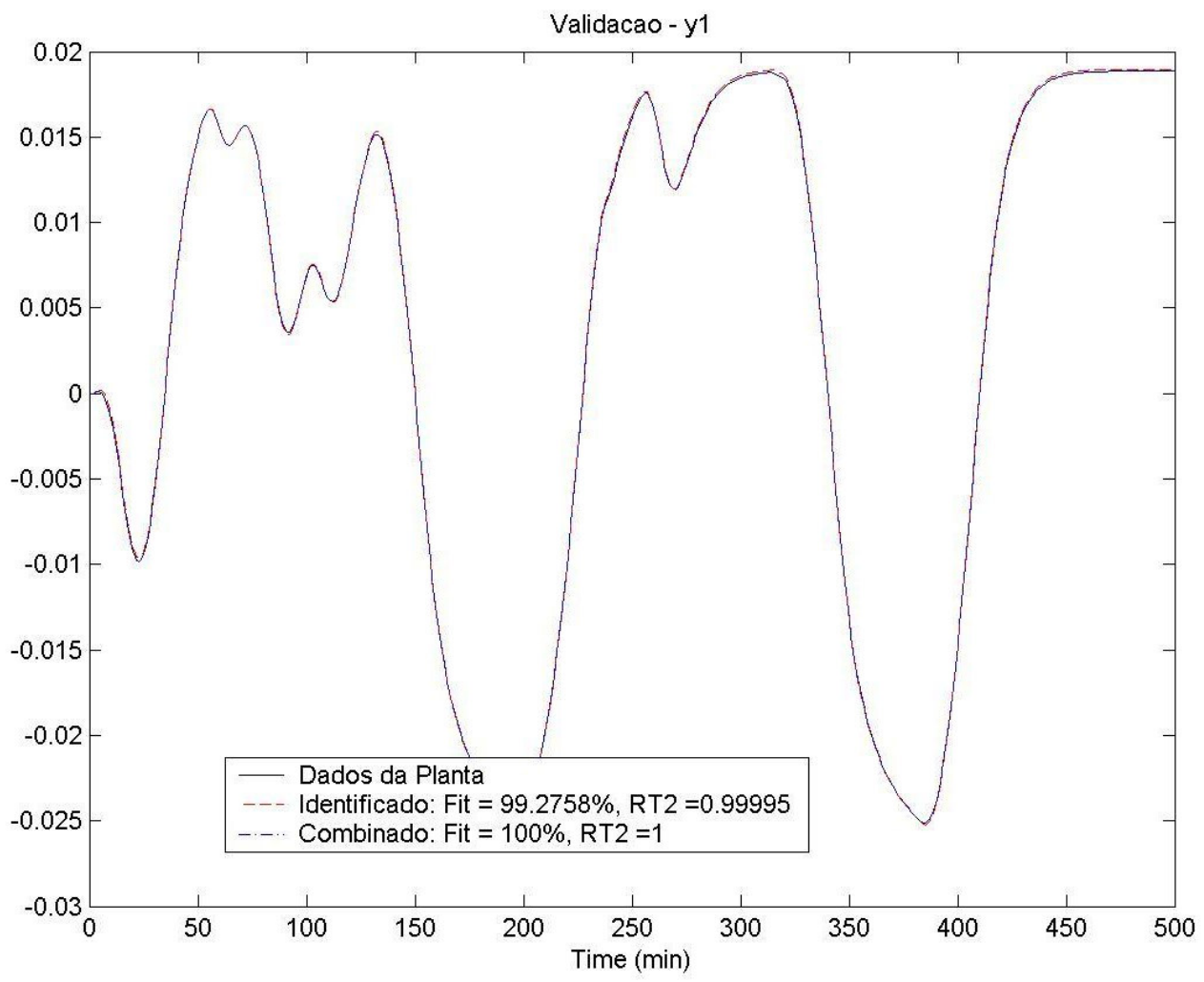

Figura 6-40 - Validação do modelo para y1 


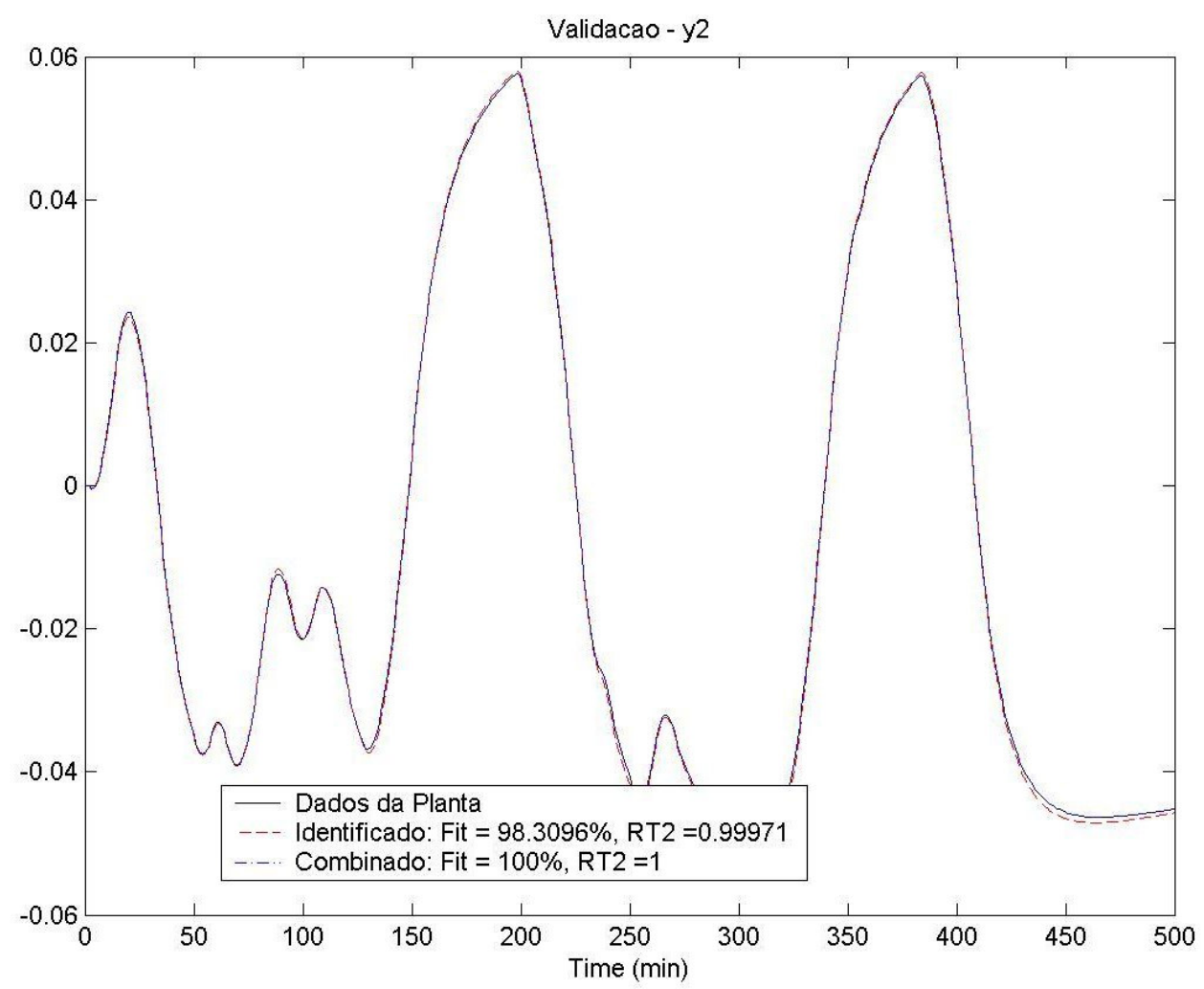

Figura 6-41 - Validação do Modelo para y2

\subsubsection{Análise dos resultados dos métodos de identificação}

Para todos os casos estudados pela metodologia de identificação, a resposta ao degrau para o modelo obtido pela metodologia de combinação de modelos usada no controle adaptativo, estava muito próxima da planta, às vezes coincidindo com a mesma.

Os valores dos coeficientes $R_{2}^{T}$ e FIT estão acima de 0.99 e 92\%, respectivamente, para os modelos obtidos pelo controlador adaptativo. Diferentemente, os coeficientes $R_{2}^{T}$ e FIT obtidos pelo caso 5, onde o modelo do controlador AQDMC não representa fielmente a planta, são respectivamente, 0.91 e 70\%.

Considerando os coeficientes $R_{2}^{T}$ e FIT, para o modelo identificado através de um modelo ARX, os valores estão acima de 0.99 e $92 \%$, respectivamente. Sendo que a resposta ao degrau para alguns destes modelos identificados estão um pouco distantes da resposta ao degrau no modelo da planta. 


\section{CONCLUSÕES}

As principais conclusões são apresentadas a seguir:

$\checkmark$ A análise dos ganhos obtidas na seção 6.2 (Estudo dos Modelos) permite concluir que os pontos de operação utilizados para o levantamento dos modelos são de regiões bem distintas fazendo com os ganhos variem consideravelmente entre os modelos.

$\checkmark$ A metodologia proposta para o controlador MPC adaptativo apresentou bons resultados. O método permite obter modelos que estão próximos, às vezes, coincidindo com o modelo da planta. Além disso, o controlador adaptativo se apresenta estável e robusto, mesmo quando o modelo inicial do controlador não é favorável a isto tal como o modelo FD.

$\checkmark$ As diferentes combinações dos coeficientes $\lambda_{i}$ obtidas ao longo do mesmo experimento para uma mesma planta mostra que o método numérico utilizado para resolver o problema de otimização para a obtenção dos coeficientes $\lambda_{i}$ não foi capaz de convergir sempre para o modelo da planta.

$\checkmark$ O AQDMC apresentou resposta mais rápida quando comparado com o MMPC para os casos estudados na Validação da Metodologia.

$\checkmark$ O AQDMC foi capaz de manter o processo sob controle mesmo quando o modelo obtido pela combinação está relativamente distante do modelo da planta conforme verificado pelo Caso 5 da seção de validação. Entretanto, para este caso, deve ser considerado a re-sintonia do controlador para obter uma melhor resposta.

$\checkmark$ Os autovalores da matriz de excitação $\left(\bar{A}^{T} \bar{A}\right)$ obtidos nos experimentos são diferentes de zero, mostrando que o grau de excitação para a obtenção do modelo pelo AQDMC foi suficiente para a identificação dos coeficientes $\lambda_{i}$.

$\checkmark$ Mesmo durante o processo de excitação, verifica-se que as variáveis controladas permanecem dentro das faixas de controle minimizando o sinal de feedback 
gerado pelo controlador. Isso mostra que a forma de excitação através da camada de otimização e a adoção de controle por faixas é bastante adequado para ser adotado na prática.

$\checkmark$ Realizando a comparação entre o AQDMC e o controlador MMPC verifica-se que os resultados obtidos com os controladores adaptativos são melhores. Uma vantagem verificada pela metodologia proposta é que a resposta às perturbações é mais rápida do que a do MMPC.

$\checkmark$ A validação através da identificação ARX demonstrou que os modelos obtidos pelo AQDMC se aproximam mais da planta do que os modelos obtidos através de identificação $A R X$ tanto pelos testes degraus quanto pela simulação. Desta forma, o controlador AQDMC identificou melhores modelos do que a própria metodologia de ARX.

$\checkmark$ Também é possível concluir que o tempo de experimento necessário para o controlador AQDMC é menor quando comparado com os trabalhos de Conner e Seborg (2004) e Sotomayor et al. (2008). 


\section{CONTINUIDADE DO TRABALHO}

Devido ao progresso das técnicas de identificação para controles MPC, os controladores multimodelos despontam como uma área promissora e que deve continuar sendo estudada. Assim, seguem alguns pontos que podem ser focados em trabalhos posteriores a este:

> Estudar a metodologia do controlador adaptativo com excitação externa para modelos lineares de outros processos.

> Considerar o estudo da metodologia do controlador adaptativo, também, para processos representados por modelos não lineares.

> Desenvolvimento de regras para disparar a identificação de um novo modelo para o controlador adaptativo. Estas regras poderiam disparar rotinas automáticas de identificação.

$>$ Estudar os parâmetros de sintonia de tal forma a se ter uma tabela relacionando os modelos identificados a suas melhores sintonias.

> Aplicação do controlador adaptativo estudado neste trabalho em um processo real. 


\section{REFERÊNCIAS BIBLIOGRÁFICAS}

ALMEIDA, E.; RODRIGUES, M. A.; ODLOAK D. (2000) Robust predictive control of a gasoline debutanizer column. Brazilian Journal of Chemical Engineering, v.17, n0407, p.967-977.

ALMEIDA, E.; ZANIN, A. C.; ODLOAK, D. (1996). Advanced control and of a crude vacuum unit. XI Congresso Brasileiro de Automática, - I Simpósio de Automática Aplicada, Anais p.23-28, São Paulo.

BALLIN, S. L. 2008. Controlador Preditivo Multivariável com Restrição de Excitação para Identificação de Processos em Malha Fechada. 128p. Dissertação (Mestrado) Escola Politécnica da Universidade de São Paulo.

BEZERRA, V.M., ABU-EL-ZEET, Z.H. e ROBERTS, P.D. (1999). Integrating predictive control and economic optimization. Computing and Control Engineering Journal, 10(5): 198-208.

BOLZAN, A. (1991) - Ajuste e Implementação de um Controlador Adaptativo para um Reator Semi-Batelada, Tese de Doutorado, COPPE, Universidade Federal do Rio de Janeiro - UFRJ, Rio de Janeiro.

CLARKE, D. W.; MOHTADI, C.; TUFFS, P. S. (1987). Generalized predictive controlPart I: the basic algorithm. Automatica, v.23, n.2, p.137-148.

CLARKE, D. W.; MOHTADI, C.; TUFFS, P. S. (1987). Generalized predictive controlPart II: extensions and interpretations. Automatica, v.23, n.2, p.149-160.

CONNER, J.S.; SEBORG, D.E. (2004). An evaluation of MIMO input designs for process identification. Industrial and Engineering Chemistry Research, 43(14): 38473854 .

CUTLER, C. R.; RAMAKER, B. L. (1979). Dynamic matrix control - a computer control algorithm. AIChE 86th National Meeting, Houton, TX.

CUTLER, C. R. (1983). Dynamic matrix control - an Optimal Multivariable Control Algorithm with Constraints. Houston. PhD Thesis - University of Houston. 
GARNIER, H.; GILSON, M.; CERVELLIN, O. (2006) Latest developments for the Matlab CONTSID toolbox. In: 14th IFAC SYMPOSIUM ON SYSTEM IDENTIFICATION (SYSID 2006), 2006, Newcastle, Australia. Anais. CD-ROM.

GARCIA, C. E.; MORSHEDI, A. M. (1986). Quadratic programming solution of dynamic matrix control (QDMC). Chem. Engng Commun., v.46, p.73087.

GARCIA, C. (2006) Identificação de sistemas. Notas de aula. Universidade de São Paulo.

HENSON, M. A. (1998). Nonlinear model predictive control: Current status and fucture directions. Computers Chem. Engng, v.23, n.2, p.187-202.

HOYD, M.; LEE, J. H.; MORARI, M. (1993). Truncated step response model predictive control. Journal of Process Control, v.3, n.2, p.67-73.

KOTHARE, K. V.; BALAKRISHNAN, V.; MORARI, M. (1996). Robust constrained model predictive control using linear matrix inequalities. Automatica, v.30, n.10, p.1361-1379.

KANELLAKOPOULOS, I., KOKOTOVIC, P.V., MARINO, R. (1991) - An Extended Direct Scheme for Robust Adaptive Nonlinear Control, Automatica, v. 27, $\mathrm{N}^{\circ} 2, \mathrm{p}$. $247-255$.

LANDAU, I.D. (2001). Identification in Closed Loop: a Powerful Design tool (better design models, simpler controllers). Control Engineering Practice, 9(1):p.51-65.

LEE, J. H.; MORARI, M.; GARCIA, C.E. (1994). State space interpretation of model predictive control, Automatica, v.30, n.4, p.707-717.

LI, S.; LIM, K. Y.; FISHER, D. G. (1989). A state space formulation for model predictive control, AIChE Journal, v.35, n.2, p.2, p.241-249.

LJUNG, L. (1999). System Identification: Theory for the user. Prentice-Hall PTR, NJ, $2^{\text {nd }}$ edition.

LUNDSTRÔM, P.; LEE, J. H.;MORARI, M.; SKOGESTAD, S. (1995). Limitations of dynamic matrix control. Computers Chem. Engng, v.19, n.4, p.409-421. 
MACIEJOWSKI, J.M. (2002) Predictive Control with Constraints - Prentice Hall.

MAITI, S. N.; SARAF, D. N. (1995) Adaptive Dynamic Matriz Control of a Distillation Column eith Closed-Loop Online Identification. Journal of Process Control, v.5, p. 315-327.

MAYNE, D. Q.; RAWLINGS, J. B.; RAO, C. V.; SCOKAERT, P. M. Constrained Model Predictive Control: Stability and Optimality. Automatica, Vol. 36, p. 789-814, 2000

MIRANDA, F. C. P. R. (2005). Identificação de Sistemas em Malha Fechada usando Controlador Preditivo Multivariável: um Caso Industrial. 99p. Dissertação (Mestrado) - Escola Politécnica da Universidade de São Paulo.

MORARI, M.; DOYLE, J. (1986). A unifying frameworks for control system design under uncertainty and its implications for chemical process control. Proceedings of Chemical Process Control - CPC III, p.5-52

MORARI, M.; LEE, J. H. (1999). Model predictive control: past, present and future. Computers Chem. Engng, v.23, p.667-682.

MORARI, M.; ZAFIRIOU, E. (1989). Robust process control. Prentice-Hall, Englewood Cliffs, NJ.

MORO, L. F. L.; ODLOAK, D. (1995). Constrained multivariable control of fluid cracking converters. Journal of Process Control, v.5, n.1, p.1, p.29-39.

MORSHEDI, A. M.; CUTLER, C. R.; SKROVANEK, T. A. (1985). Optimal solution of dynamic matrix control with linear programming techniques (LDMC). Proc. Am. Control Conf., Boston, MA, p.199-208.

MUNIZ, L. A. R. (2004) Controle Preditivo Adaptativo Aplicado a um Reator de Pirólise Operando em Regime Semi-Batelada. 133p. Dissertação (Doutorado) Universidade Federal de Santa Catarina.

ODLOAK, D. (1995). Stability analysis of the dynamic matrix controller, Braz. Jour. Chem. Engng, v.12, n.1, p.50-64. 
ODLOAK, D. (1996). A new state-space approach to model predictive control, Braz. Jour. Chem. Engng, v.13, n.3, p.152-167.

ODLOAK, D. (2005). Model Predictive Control (MPC). Lecture notes, Polytechnic School of the University of São Paulo Brazil.

PORFÍRIO, C. R. 2001. Implantação de um Controlador Multimodelos em uma Coluna Depropanizadora Industrial. 130p. Dissertação (Mestrado) - Escola Politécnica da Universidade de São Paulo.

PORFÍRIO, C.R.; ALMEIDA NETO, E.;ODLOAK, D. (2003) Multi-model Predictive Control of an industrial C3/C4 Splitter. Control Engineering Practice. 11(7), 765-779.

PRETT, D. M.; GILLETTE, R. D. (1980). Optimization and constrained multivariable control of catalytic cracking unit, Proc. Automatic Control Conf., San Francisco , CA, Paper WP5-C.

RICHALET, J.; RAULT, A.; TESTUD, J. L.; PAPON, J. (1978). Model predictive heuristic control: application to industrial processes. Automatica, v. 14, n.5, p.413428.

RODRIGUES, M. A.; ODLOAK, D. (2000). Output feedback MPC with guaranteed robust stability. Journal of Process Control, v.10, p. 557-572.

RODRIGUES, M. A.; ODLOAK, D. (2002).MPC for stable linear systems with model uncertainty. Automatica, v.39, p. 569-583.

RODRIGUES, M. A. \& ODLOAK, D (2005). Robust MPC for systems with output feedback and input saturation. Journal of Process Control 15, p. 837-846.

ROTAVA, O. e ZANIN A.C. (2005). Multivariable control and real-time optimization an industrial practical view. Hydrocarbon Processing, 84(6): 61-71.

ROUHANI, R.; MEHRA, R. K. (1982). Model algorithm control (MAC): basic theoretical properties, Automatica, v.18, p.401. 
ROVITHAKIS, G.A., CHRISTODOULOU, M.A. (1994) - Adaptive Control of Unknown Plants Using Dynamical Neural Networks, IEE Transactions on Systems, Man, and Cybernetics, v. 24, n. 3.

SEBORG, D., EDGAR, T.F., DUNCAN, A.M. (1989) Process Dynamics and Control, New York: John Wiley \& Sons.

SOTOMAYOR, O. A. Z., ODLOAK, D and MORO, F. L. (2008) Closed-loop model reidentification of processes under MPC with zone control, Control Engineering Practice. DOI: 10.1016/j.conengprac.2008.10.002.

SPANDRI, R.; MORO, L. F. L.; ODLOAK, D. (1995). Constranined multivarible control of fluid catalytic cracking converters, a practical application. AIChE, Spring National Meeting, Paper 84b, Houston, TX.

TVRZSKÁ de GOUVÊIA, M., ODLOAK, D. (1997). ROSSMPC: A new way of representing and analyzing predictive controllers. Chemical and Engineering Research Design, 75, 693-709.

ZANIN, A.C. (2001). Implementação industrial de um otimizador em tempo real. Tese de Doutorado, Departamento de Engenharia Química, Escola Politécnica da Universidade de São Paulo.

ZANIN, A.C.; ODLOAK, D. (1992). Advanced control of an atmospheric resid heater. Proceedings of the First International Instrumentation, System and Industrial Automation Meeting, p.33-42, Salvador, BA.

ZHU, Y (2001). Multivariable System Identification for Process Control PERGAMON. 


\section{Anexo A - Modelos Coluna Depropanizadora}

Os modelos obtidos por Porfírio (2001) são apresentados nas tabelas a seguir. Nestas tabelas são apresentados os coeficientes da função de transferência na forma $G(s)=\frac{b_{0}+b_{1} s}{s^{2}+a_{1} s+a_{2}} e^{-s \theta}($ A.1)

Tabela A-1 - Coeficientes Modelo 1

\begin{tabular}{|l|l|l|l|l|l|l|}
\hline $\mathrm{CV}$ & $\mathrm{MV} / \mathrm{DV}$ & $\mathrm{b}_{0}$ & $\mathrm{~b}_{1}$ & $\mathrm{a}_{1}$ & $\mathrm{a}_{2}$ & $\Theta$ \\
\hline $\mathrm{C}_{3}{ }^{-}$ & $\mathrm{V}_{\mathrm{RF}}$ & $1.09 \mathrm{e}-05$ & $4.23 \mathrm{e}-05$ & $1.09 \mathrm{e}-01$ & $2.43 \mathrm{e}-02$ & 1 \\
\hline & $\mathrm{V}_{\text {GOP }}$ & $-3.82 \mathrm{e}-05$ & $-1.21 \mathrm{e}-04$ & $1.34 \mathrm{e}-01$ & $1.11 \mathrm{e}-02$ & 1 \\
\hline & $\mathrm{V}_{\text {CARGA }}$ & $5.67 \mathrm{e}-03$ & $-9.40 \mathrm{e}-04$ & $3.98 \mathrm{e}-01$ & $8.50 \mathrm{e}-02$ & 1 \\
\hline & $\mathrm{T}_{\text {GOP }}$ & $-2.17 \mathrm{e}-04$ & $-6.86 \mathrm{e}-04$ & $1.34 \mathrm{e}-01$ & $1.11 \mathrm{e}-02$ & 1 \\
\hline $\mathrm{C}_{4}{ }^{+}$ & $\mathrm{V}_{\mathrm{RF}}$ & $-2.54 \mathrm{e}-04$ & $-2.45 \mathrm{e}-04$ & $1.14 \mathrm{e}-01$ & $8.60 \mathrm{e}-03$ & 1 \\
\hline & $\mathrm{V}_{\text {GOP }}$ & $6.64 \mathrm{e}-04$ & $7.16 \mathrm{e}-04$ & $1.34 \mathrm{e}-01$ & $8.70 \mathrm{e}-03$ & 1 \\
\hline & $\mathrm{V}_{\text {CARGA }}$ & $-2.48 \mathrm{e}-04$ & $6.17 \mathrm{e}-05$ & $1.57 \mathrm{e}-01$ & $1.41 \mathrm{e}-02$ & 1 \\
\hline & $\mathrm{T}_{\text {GOP }}$ & $9.96 \mathrm{e}-03$ & $1.07 \mathrm{e}-02$ & $1.34 \mathrm{e}-01$ & $8.70 \mathrm{e}-03$ & 1 \\
\hline $\mathrm{T}_{\text {TOPO }}$ & $\mathrm{V}_{\text {RF }}$ & $-1.12 \mathrm{e}-04$ & $-8.73 \mathrm{e}-05$ & $1.32 \mathrm{e}-01$ & $7.30 \mathrm{e}-03$ & 1 \\
\hline & $\mathrm{V}_{\text {GOP }}$ & $7.00 \mathrm{e}-03$ & $1.30 \mathrm{e}-03$ & $2.26 \mathrm{e}+00$ & $1.37 \mathrm{e}-01$ & 1 \\
\hline & $\mathrm{V}_{\text {CARGA }}$ & $-1.50 \mathrm{e}-03$ & $-4.00 \mathrm{e}-04$ & $1.92 \mathrm{e}+00$ & $1.35 \mathrm{e}-01$ & 1 \\
\hline & $\mathrm{T}_{\text {GOP }}$ & $1.04 \mathrm{e}-01$ & $1.94 \mathrm{e}-02$ & $2.26 \mathrm{e}+00$ & $1.37 \mathrm{e}-01$ & 1 \\
\hline
\end{tabular}


Tabela A-2 - Coeficientes Modelo 2

\begin{tabular}{|l|l|l|l|l|l|l|}
\hline CV & MV/DV & b0 & b1 & a1 & a2 & $\Theta$ \\
\hline $\mathrm{C}_{3}{ }^{-}$ & $\mathrm{V}_{\mathrm{RF}}$ & $4.22 \mathrm{e}-04$ & $-2.72 \mathrm{e}-04$ & $1.66 \mathrm{e}+00$ & $2.53 \mathrm{e}-01$ & 1 \\
\hline & $\mathrm{V}_{\text {GOP }}$ & $-1.41 \mathrm{e}-04$ & $-2.18 \mathrm{e}-04$ & $1.32 \mathrm{e}-01$ & $1.17 \mathrm{e}-02$ & 1 \\
\hline & $\mathrm{V}_{\text {CARGA }}$ & $5.21 \mathrm{e}-05$ & $1.50 \mathrm{e}-04$ & $2.10 \mathrm{e}-01$ & $2.79 \mathrm{e}-02$ & 1 \\
\hline & $\mathrm{T}_{\text {GOP }}$ & $-4.74 \mathrm{e}-04$ & $-7.37 \mathrm{e}-04$ & $1.32 \mathrm{e}-01$ & $1.17 \mathrm{e}-02$ & 1 \\
\hline $\mathrm{C}_{4}{ }^{+}$ & $\mathrm{V}_{\mathrm{RF}}$ & $-2.89 \mathrm{e}-03$ & $-1.57 \mathrm{e}-03$ & $7.36 \mathrm{e}-01$ & $5.61 \mathrm{e}-02$ & 1 \\
\hline & $\mathrm{V}_{\text {GOP }}$ & $9.46 \mathrm{e}-04$ & $1.61 \mathrm{e}-03$ & $1.27 \mathrm{e}-01$ & $1.23 \mathrm{e}-02$ & 1 \\
\hline & $\mathrm{V}_{\text {CARGA }}$ & $-3.89 \mathrm{e}-04$ & $-3.83 \mathrm{e}-04$ & $1.36 \mathrm{e}-01$ & $1.48 \mathrm{e}-02$ & 1 \\
\hline & $\mathrm{T}_{\text {GOP }}$ & $1.22 \mathrm{e}-02$ & $2.06 \mathrm{e}-02$ & $1.27 \mathrm{e}-01$ & $1.23 \mathrm{e}-02$ & 1 \\
\hline $\mathrm{T}_{\text {TOPO }}$ & $\mathrm{V}_{\mathrm{RF}}$ & $-6.30 \mathrm{e}-03$ & $-3.40 \mathrm{e}-03$ & $2.07 \mathrm{e}+00$ & $2.43 \mathrm{e}-01$ & 1 \\
\hline & $\mathrm{V}_{\text {GOP }}$ & $4.50 \mathrm{e}-03$ & $2.00 \mathrm{e}-04$ & $8.35 \mathrm{e}-01$ & $8.12 \mathrm{e}-02$ & 1 \\
\hline & $\mathrm{V}_{\text {CARGA }}$ & $-1.20 \mathrm{e}-03$ & $-1.20 \mathrm{e}-03$ & $8.33 \mathrm{e}-01$ & $6.93 \mathrm{e}-02$ & 1 \\
\hline & $\mathrm{T}_{\text {GOP }}$ & $5.75 \mathrm{e}-02$ & $2.00 \mathrm{e}-03$ & $8.35 \mathrm{e}-01$ & $8.12 \mathrm{e}-02$ & 1 \\
\hline
\end{tabular}

Tabela A-3 - Coeficientes Modelo 3

\begin{tabular}{|l|l|l|l|l|l|l|}
\hline $\mathrm{CV}$ & $\mathrm{MV} / \mathrm{DV}$ & $\mathrm{b}_{0}$ & $\mathrm{~b}_{1}$ & $\mathrm{a}_{1}$ & $\mathrm{a}_{2}$ & $\Theta$ \\
\hline $\mathrm{C}_{3}{ }^{-}$ & $\mathrm{V}_{\mathrm{RF}}$ & $1.53 \mathrm{e}-03$ & $-8.60 \mathrm{e}-04$ & $1.19 \mathrm{e}+00$ & $9.12 \mathrm{e}-02$ & 1 \\
\hline & $\mathrm{V}_{\text {GOP }}$ & $-7.81 \mathrm{e}-04$ & $-3.77 \mathrm{e}-04$ & $3.40 \mathrm{e}-01$ & $1.81 \mathrm{e}-02$ & 1 \\
\hline & $\mathrm{V}_{\text {CARGA }}$ & $7.70 \mathrm{e}-04$ & $8.93 \mathrm{e}-04$ & $5.63 \mathrm{e}-01$ & $3.59 \mathrm{e}-02$ & 1 \\
\hline & $\mathrm{T}_{\text {GOP }}$ & $-7.70 \mathrm{e}-03$ & $-3.72 \mathrm{e}-03$ & $-3.40 \mathrm{e}-01$ & $1.81 \mathrm{e}-02$ & 1 \\
\hline $\mathrm{C}_{4}{ }^{+}$ & $\mathrm{V}_{\mathrm{RF}}$ & $-1.05 \mathrm{e}-03$ & $-2.64 \mathrm{e}-03$ & $3.52 \mathrm{e}-01$ & $2.31 \mathrm{e}-02$ & 1 \\
\hline & $\mathrm{V}_{\text {GOP }}$ & $1.34 \mathrm{e}-03$ & $4.74 \mathrm{e}-04$ & $2.47 \mathrm{e}-01$ & $2.03 \mathrm{e}-02$ & 1 \\
\hline & $\mathrm{V}_{\text {CARGA }}$ & $-3.30 \mathrm{e}-04$ & $-2.00 \mathrm{e}-04$ & $2.29 \mathrm{e}-01$ & $1.90 \mathrm{e}-02$ & 1 \\
\hline & $\mathrm{T}_{\text {GOP }}$ & $1.32 \mathrm{e}-02$ & $4.67 \mathrm{e}-03$ & $2.47 \mathrm{e}-01$ & $2.03 \mathrm{e}-02$ & 1 \\
\hline & $\mathrm{V}_{\text {RF }}$ & $-8.00 \mathrm{e}-04$ & $-3.40 \mathrm{e}-03$ & $4.02 \mathrm{e}-01$ & $3.65 \mathrm{e}-02$ & 1 \\
\hline & $V_{\text {GOP }}$ & $8.90 \mathrm{e}-03$ & $6.40 \mathrm{e}-03$ & $1.90 \mathrm{e}+00$ & $1.95 \mathrm{e}-01$ & 1 \\
\hline & $V_{\text {CARGA }}$ & $-9.00 \mathrm{e}-04$ & $-1.20 \mathrm{e}-03$ & $8.84 \mathrm{e}-01$ & $7.99 \mathrm{e}-02$ & 1 \\
\hline & $\mathrm{T}_{\text {GOP }}$ & $8.77 \mathrm{e}-02$ & $6.34 \mathrm{e}-02$ & $1.90 \mathrm{e}+00$ & $1.95 \mathrm{e}-01$ & 1 \\
\hline
\end{tabular}


Tabela A-4 - Coeficientes Modelo 4

\begin{tabular}{|l|l|l|l|l|l|l|}
\hline $\mathrm{CV}$ & $\mathrm{MV} / \mathrm{DV}$ & $\mathrm{b}_{0}$ & $\mathrm{~b}_{1}$ & $\mathrm{a}_{1}$ & $\mathrm{a}_{2}$ & $\Theta$ \\
\hline $\mathrm{C}_{3}{ }^{-}$ & $\mathrm{V}_{\mathrm{RF}}$ & $4.88 \mathrm{e}-04$ & $-1.11 \mathrm{e}-04$ & $9.88 \mathrm{e}-01$ & $6.46 \mathrm{e}-02$ & 1 \\
\hline & $\mathrm{V}_{\text {GOP }}$ & $-1.86 \mathrm{e}-04$ & $-1.76 \mathrm{e}-04$ & $2.61 \mathrm{e}-01$ & $9.10 \mathrm{e}-03$ & 1 \\
\hline & $\mathrm{V}_{\text {CARGA }}$ & $2.71 \mathrm{e}-04$ & $1.85 \mathrm{e}-04$ & $5.11 \mathrm{e}-01$ & $2.65 \mathrm{e}-02$ & 1 \\
\hline & $\mathrm{T}_{\text {GOP }}$ & $-1.78 \mathrm{e}-03$ & $-1.69 \mathrm{e}-03$ & $2.61 \mathrm{e}-01$ & $9.10 \mathrm{e}-03$ & 1 \\
\hline $\mathrm{C}_{4}{ }^{+}$ & $\mathrm{V}_{\mathrm{RF}}$ & $-4.40 \mathrm{e}-04$ & $-1.96 \mathrm{e}-03$ & $1.51 \mathrm{e}-01$ & $7.40 \mathrm{e}-03$ & 1 \\
\hline & $\mathrm{V}_{\text {GOP }}$ & $5.08 \mathrm{e}-04$ & $-3.73 \mathrm{e}-04$ & $1.04 \mathrm{e}-01$ & $4.60 \mathrm{e}-03$ & 1 \\
\hline & $\mathrm{V}_{\text {CARGA }}$ & $-1.86 \mathrm{e}-04$ & $-3.43 \mathrm{e}-04$ & $9.67 \mathrm{e}-02$ & $4.60 \mathrm{e}-03$ & 1 \\
\hline & $\mathrm{T}_{\text {GOP }}$ & $4.86 \mathrm{e}-03$ & $-3.57 \mathrm{e}-03$ & $1.04 \mathrm{e}-01$ & $4.60 \mathrm{e}-03$ & 1 \\
\hline $\mathrm{T}_{\text {TOPO }}$ & $\mathrm{V}_{\text {RF }}$ & $-2.50 \mathrm{e}-03$ & $-3.90 \mathrm{e}-03$ & $8.87 \mathrm{e}-01$ & $8.40 \mathrm{e}-02$ & 1 \\
\hline & $\mathrm{V}_{\text {GOP }}$ & $2.90 \mathrm{e}-03$ & $5.50 \mathrm{e}-03$ & $8.60 \mathrm{e}-01$ & $3.92 \mathrm{e}-02$ & 1 \\
\hline & $\mathrm{V}_{\text {CARGA }}$ & $-1.80 \mathrm{e}-03$ & $-4.00 \mathrm{e}-04$ & $1.89 \mathrm{e}+00$ & $6.69 \mathrm{e}-02$ & 1 \\
\hline & $\mathrm{T}_{\text {GOP }}$ & $2.81 \mathrm{e}-02$ & $5.27 \mathrm{e}-02$ & $8.60 \mathrm{e}-01$ & $3.92 \mathrm{e}-02$ & 1 \\
\hline
\end{tabular}

Tabela A-5 - Coeficientes Modelo 5

\begin{tabular}{|l|l|l|l|l|l|l|}
\hline $\mathrm{CV}$ & $\mathrm{MV} / \mathrm{DV}$ & $\mathrm{b}_{0}$ & $\mathrm{~b}_{1}$ & $\mathrm{a}_{1}$ & $\mathrm{a}_{2}$ & $\Theta$ \\
\hline $\mathrm{C}_{3}{ }^{-}$ & $\mathrm{V}_{\mathrm{RF}}$ & $5.65 \mathrm{e}-04$ & $-3.54 \mathrm{e}-04$ & $8.17 \mathrm{e}-01$ & $8.09 \mathrm{e}-02$ & 1 \\
\hline & $\mathrm{V}_{\text {GOP }}$ & $-4.78 \mathrm{e}-04$ & $-1.43 \mathrm{e}-04$ & $3.42 \mathrm{e}-01$ & $2.59 \mathrm{e}-02$ & 1 \\
\hline & $\mathrm{V}_{\text {CARGA }}$ & $6.79 \mathrm{e}-04$ & $-1.15 \mathrm{e}-04$ & $9.00 \mathrm{e}-01$ & $7.12 \mathrm{e}-02$ & 1 \\
\hline & $\mathrm{T}_{\text {GOP }}$ & $-6.48 \mathrm{e}-03$ & $-1.94 \mathrm{e}-03$ & $3.42 \mathrm{e}-01$ & $2.59 \mathrm{e}-02$ & 1 \\
\hline $\mathrm{C}_{4}{ }^{+}$ & $\mathrm{V}_{\mathrm{RF}}$ & $-2.86 \mathrm{e}-03$ & $-1.21 \mathrm{e}-03$ & $1.17 \mathrm{e}+00$ & $7.16 \mathrm{e}-02$ & 1 \\
\hline & $\mathrm{V}_{\text {GOP }}$ & $1.35 \mathrm{e}-03$ & $1.30 \mathrm{e}-03$ & $3.25 \mathrm{e}-01$ & $1.80 \mathrm{e}-02$ & 1 \\
\hline & $\mathrm{V}_{\text {CARGA }}$ & $-4.58 \mathrm{e}-04$ & $2.03 \mathrm{e}-04$ & $3.57 \mathrm{e}-01$ & $1.73 \mathrm{e}-02$ & 1 \\
\hline & $\mathrm{T}_{\text {GOP }}$ & $1.83 \mathrm{e}-02$ & $1.77 \mathrm{e}-02$ & $3.25 \mathrm{e}-01$ & $1.80 \mathrm{e}-02$ & 1 \\
\hline $\mathrm{T}_{\text {TOPO }}$ & $\mathrm{V}_{\text {RF }}$ & $-2.10 \mathrm{e}-03$ & $-1.90 \mathrm{e}-03$ & $1.17 \mathrm{e}+00$ & $1.07 \mathrm{e}-01$ & 1 \\
\hline & $\mathrm{V}_{\text {GOP }}$ & $8.10 \mathrm{e}-03$ & $5.30 \mathrm{e}-03$ & $2.42 \mathrm{e}+00$ & $1.76 \mathrm{e}-01$ & 1 \\
\hline & $\mathrm{V}_{\text {CARGA }}$ & $-7.62 \mathrm{e}-04$ & $-5.32 \mathrm{e}-04$ & $9.82 \mathrm{e}-01$ & $5.49 \mathrm{e}-02$ & 1 \\
\hline & $\mathrm{T}_{\text {GOP }}$ & $7.66 \mathrm{e}-02$ & $7.25 \mathrm{e}-02$ & $1.67 \mathrm{e}+00$ & $1.24 \mathrm{e}-01$ & 1 \\
\hline
\end{tabular}


Tabela A-6 - Coeficientes Modelo FD

\begin{tabular}{|l|l|l|l|l|l|l|}
\hline $\mathrm{CV}$ & $\mathrm{MV} / \mathrm{DV}$ & $\mathrm{b}_{0}$ & $\mathrm{~b}_{1}$ & $\mathrm{a}_{1}$ & $\mathrm{a}_{2}$ & $\Theta$ \\
\hline $\mathrm{C}_{3}{ }^{-}$ & $\mathrm{V}_{\mathrm{RF}}$ & $5.66 \mathrm{e}-04$ & $-2.22 \mathrm{e}-04$ & $3.49 \mathrm{e}+00$ & $5.90 \mathrm{e}-01$ & 1 \\
\hline & $\mathrm{V}_{\text {GOP }}$ & $-1.45 \mathrm{e}-03$ & $7.41 \mathrm{e}-05$ & $2.70 \mathrm{e}+00$ & $4.02 \mathrm{e}-01$ & 1 \\
\hline & $\mathrm{V}_{\text {CARGA }}$ & $1.13 \mathrm{e}-04$ & $-1.14 \mathrm{e}-04$ & $4.56 \mathrm{e}-01$ & $3.23 \mathrm{e}-02$ & 1 \\
\hline & $\mathrm{T}_{\text {GOP }}$ & $-1.64 \mathrm{e}-02$ & $-1.32 \mathrm{e}-02$ & $1.44 \mathrm{e}+00$ & $1.84 \mathrm{e}-01$ & 1 \\
\hline $\mathrm{C}_{4}{ }^{+}$ & $\mathrm{V}_{\mathrm{RF}}$ & -- & -- & -- & -- & 1 \\
\hline & $\mathrm{V}_{\text {GOP }}$ & -- & -- & -- & -- & 1 \\
\hline & $\mathrm{V}_{\text {GARGA }}$ & -- & -- & -- & -- & 1 \\
\hline & $\mathrm{T}_{\text {GOP }}$ & -- & -- & -- & -- & 1 \\
\hline $\mathrm{T}_{\text {TOPO }}$ & $\mathrm{V}_{\text {RF }}$ & $-1.24 \mathrm{e}-03$ & $-1.14 \mathrm{e}-03$ & $1.64 \mathrm{e}+00$ & $9.85 \mathrm{e}-02$ & 1 \\
\hline & $\mathrm{V}_{\text {GOP }}$ & $2.00 \mathrm{e}-03$ & $-3.00 \mathrm{e}-04$ & $2.43 \mathrm{e}+00$ & $6.51 \mathrm{e}-02$ & 1 \\
\hline & $\mathrm{V}_{\text {CARGA }}$ & $-3.83 \mathrm{e}-04$ & $1.72 \mathrm{e}-04$ & $2.30 \mathrm{e}-01$ & $2.63 \mathrm{e}-02$ & 1 \\
\hline & $\mathrm{T}_{\text {GOP }}$ & $6.97 \mathrm{e}-03$ & $2.00 \mathrm{e}-03$ & $1.73 \mathrm{e}-01$ & $1.05 \mathrm{e}-02$ & 1 \\
\hline
\end{tabular}

